\title{
DESIGN AND DEVELOPMENT OF A CATHETER-BASED TUNABLE DEVICE FOR PERCUTANEOUS MITRAL REPAIR THROUGH CORONARY SINUS
}

\author{
by \\ Ata Taghipour \\ M.Sc., Electrical Engineering, University of Tabriz, Iran, 2012 \\ B.Sc., Electrical Engineering, University of Tabriz, Iran, 2010
}

\author{
A dissertation \\ presented to Ryerson University \\ in partial fulfillment of the \\ requirements for the degree of \\ Doctor of Philosophy \\ in the program of \\ Mechanical and Industrial Engineering
}

Toronto, Ontario, Canada, 2017

(C) Ata Taghipour, 2017 


\section{Author's Declaration}

I hereby declare that I am the sole author of this dissertation. This is a true copy of the dissertation, including any required final revisions, as accepted by my examiners. I authorize Ryerson University to lend this dissertation to other institutions or individuals for the purpose of scholarly research.

I further authorize Ryerson University to reproduce this dissertation by photocopying or by other means, in total or in part, at the request of other institutions or individuals for the purpose of scholarly research.

I understand that my dissertation may be made electronically available to the public. 


\section{Abstract}

DESIGN AND DEVELOPMENT OF A CATHETER-BASED TUNABLE DEVICE FOR PERCUTANEOUS MITRAL REPAIR THROUGH CORONARY SINUS

Doctor of Philosophy, 2017

Ata Taghipour

Mechanical and Industrial Engineering, Ryerson University

According to the American Heart Association, the cardiac disease accounts for over 800,000 deaths every year (1 of every 3 deaths) in the US alone. Mitral regurgitation, which occurs in $2 \%$ of the population, has become the dominant valvular disease contributing to the high death rate caused by cardiac disease. The existing percutaneous treatments of mitral regurgitation suffer from compression of left circumflex artery, limiting their performance and causing serious iatrogenic consequences. Moreover, they are not tunable resulting in limited functionality.

In this thesis, a catheter-based tunable device is designed to be implanted inside the coronary sinus for improving mitral regurgitation grade while minimizing the applied force on the left circumflex artery. A comprehensive computed tomography scan image analysis and experiments are performed to extract the required information for the design of the device and its evaluation with FEM simulations. A new effective 
engagement mechanism for integrating the device with the steerable catheter is designed and tested through large-scale experiments.

Additionally, a temperature insensitive force/torque sensor is designed for guiding and introducing the device. This sensor can also be used in other catheter-based devices such as cardiac ablation catheters. The sensing structure of the sensor and its sensing method are evaluated by FEM simulations and large-scale prototyping. The actual-scale prototype of the sensor is fabricated, and the experiments are performed for analyzing the static and dynamic response of the sensor and its temperature cross-sensitivity. 


\section{Acknowledgments}

I would like to express my sincere thanks to my supervisor Dr. Farrokh JanabiSharifi and my co-supervisor Dr. Asim Cheema. Their guidance and advice were invaluable for development and completion of this dissertation.

I would also like to thank my supervisory committee members, Dr. Vincent Chan, Dr. Siyuan He, Dr. Ahmad Varvani-Farahani, Dr. Soosan Beheshti and Dr. Mojtaba Ahmadi. Their constructive feedback helped me in improving my Ph.D. thesis. I also appreciate the valuable advice of Dr. Xijia Gu during my Ph.D. study. I would like to thank Devin Ostrom, Technical Officer in Robotics, Mechatronics, and Manufacturing Automation Laboratory (RMAL) at Ryerson University for his help in preparation of equipment for performing experiments of this study.

I take this opportunity to thank Dr. Shahir Hasanzadeh for his useful help and guidance during this study. I also thank Dr. Aleksandar Vakanski, Dr. Akbar Assa, and Dr. Abdul Afram. I had a great experience working with them in RMAL lab. I would also like to thank the members of RMAL lab: Patrick Keenan, Lubna Shahid, Hossein Bonyan, Yaser Ali, Giuseppe Giorgio and all other students in the lab for providing a nice and pleasant research environment.

Last but not the least, I would like to thank my parents, my sister and my brother for their love and encouragement. 


\section{Dedication}

This thesis is dedicated to my wonderful parents Mohammad and Fatemeh.

It is also dedicated to my amazing sister Nasrin and my awesome brother Sina. 


\section{Table of Contents}

Declaration

Abstract iii

List of Tables $\quad x$

List of Figures $\quad$ xii

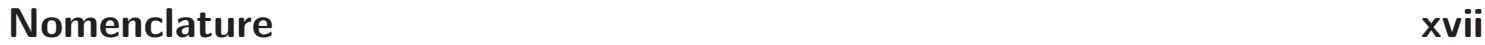

1 Introduction 1

1.1 Interventional Cardiology . . . . . . . . . . . . . . . . 1

1.2 Mitral Valve . . . . . . . . . . . . . . . . . . . . 3

1.3 Mitral Regurgitation . . . . . . . . . . . . . . 5

1.4 Treatment of MR by Interventional Cardiology . . . . . . . . . . . . . 5

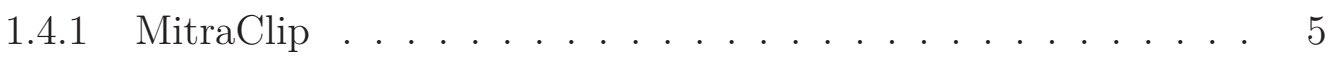

1.4.2 Monarc ..................... . . 7

1.4.3 Carillon mitral contour system . . . . . . . . . . . . . . . 9

1.4.4 Asymmetrical approach . . . . . . . . . . . . . . 10

1.4.5 Hollow tube with force applying structure . . . . . . . . . . 11

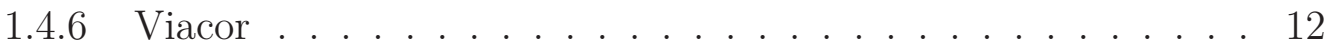

1.4.7 Comparison of existing CS approaches . . . . . . . . . 12 
1.5 Force sensor for cardiac catheters . . . . . . . . . . . . . . . . . 14

1.6 Research objectives . . . . . . . . . . . . . . . . . . . . . 18

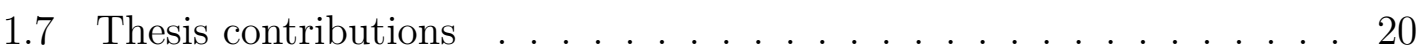

1.7.1 Design of a catheter-based device for MR treatment . . . . . . 20

1.7.2 Force sensor for cardiac catheters . . . . . . . . . . . . . 21

1.8 Thesis structure . . . . . . . . . . . . . . . . . . . . . . . 21

2 Catheter-based tunable device for percutaneous treatment of MR 23

2.1 Introduction . . . . . . . . . . . . . . . . . . . 23

2.2 Preliminary measurements . . . . . . . . . . . . . . . . 25

2.2.1 Dimensional characteristics of Coronary Sinus . . . . . . . . 25

2.2.2 Applied force to the mitral plane and the generated displacement 35

2.3 Design . . . . . . . . . . . . . . . . . . . . . . . 38

2.4 Grabbing and pulling mechanism . . . . . . . . . . . . 52

2.5 Engagement mechanism . . . . . . . . . . . . . . 54

2.6 Simulations . . . . . . . . . . . . . . . . . 57

2.6.1 FEM simulation of the device under applied force . . . . . . . 57

2.6.2 CFD simulation of blood inside CS . . . . . . . . . . . 58

2.7 Large-scale prototyping of the device . . . . . . . . . . . . . . 61

2.8 Experimental validation of engagement mechanism . . . . . . . . . 63

2.9 Summary . . . . . . . . . . . . . . . . . . . 68

3 Temperature insensitive force/torque sensor for cardiac catheters $\quad 69$

3.1 Introduction . . . . . . . . . . . . . . . . . . 69

3.2 Design of the sensing structure . . . . . . . . . . . . . . . . 69

3.3 Simulation analysis of the sensing structure . . . . . . . . . . . . . 72

3.4 Validation of the sensing structure . . . . . . . . . . . . . . 74

3.5 Sensing method . . . . . . . . . . . . . . . 78 
3.6 Sensing element . . . . . . . . . . . . . . . . . . . . . 84

3.7 Prototype of the sensor . . . . . . . . . . . . . . 85

3.8 Experimental results and discussion . . . . . . . . . . . . . 88

3.8.1 Static response of the sensor . . . . . . . . . . . . 88

3.8.2 Dynamic response of the sensor . . . . . . . . . . . . 93

3.8.3 Temperature insensitivity of the sensor . . . . . . . . . . . 94

3.9 Summary . . . . . . . . . . . . . . . . . . . . . . . 99

4 Conclusion and future directions $\quad 100$

4.1 Conclusion . . . . . . . . . . . . . . . . . . 100

4.2 Future directions . . . . . . . . . . . . . . . . . . 102

4.2.1 Catheter-based device for percutaneous treatment of MR . . . 102

4.2.2 Force sensor for cardiac catheters . . . . . . . . . . . . 103

Bibliography 


\section{List of Tables}

1.1 Mechanism of Force Applying in Different Devices . . . . . . . . . . . 13

2.1 Clinical characteristics of study population . . . . . . . . . . 28

2.2 Statistical Results from Analyses of CS Diameter and its Location with Respect to MA . . . . . . . . . . . . . . . . . 35

2.3 Applied Force for Generating Displacements at $\mathrm{P}_{1}, \mathrm{P}_{2}$ and $\mathrm{P}_{3} \ldots$. . . 38

2.4 Defining the design problem for desirable catheter-based device for MR treatment . . . . . . . . . . . . . . . . . . . 39

2.5 Morphological chart for design problem . . . . . . . . . . . . . . 40

2.6 Generated Displacement by the Links of the Device . . . . . . . . . . 52

2.7 Operation Time (seconds) for Ten People Trying to Engage Two Parts 67

3.1 Requirements of the Force Sensor for Application of Cardiac Ablation

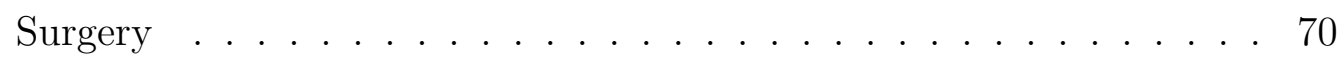

3.2 Generated Strains in FBGs under Applied Axial and Lateral Forces, Torque and Temperature Variation . . . . . . . . . . . . . 81

3.3 Sensing Structure Material Properties . . . . . . . . . . . . . 86

3.4 Bragg Wavelength, Bandwidth and Reflectivity of FBGs in Fiber 1 and Fiber $2 \ldots \ldots \ldots \ldots$. . . . . . . . . . . . . . . . 87

3.5 Maximum SD and Sensitivity for Experimental Results . . . . . . . . 92 
List of Tables

3.6 Characteristics of the Fabricated Force Sensor . . . . . . . . . . . . . 99 


\section{List of Figures}

1.1 Interventional Cardiology . . . . . . . . . . . . . . . . . . . 2

1.2 Mitral valve and its main parts . . . . . . . . . . . . . . . . . . 4

1.3 Atrial view of $\mathrm{MV} \ldots \ldots \ldots \ldots$. . . . . . . . . . . . 4

1.4 MitraClip system . . . . . . . . . . . . . . . . . . 6

1.5 CS and it location with respect to MV . . . . . . . . . 7

1.6 Monarc system . . . . . . . . . . . . . . . . . . . . 8

1.7 Carillon device . . . . . . . . . . . . . . . . . . . . . . 10

1.8 St. Jude Medical device . . . . . . . . . . . . . . . . . . . . 11

1.9 Hollow tube with force applying structure . . . . . . . . . . . . . . 12

1.10 Axial and lateral forces applied to the tip of an ablation catheter . . . 14

2.1 The relationship between different items of the chapter . . . . . . . . 24

2.2 (a) Schematic atrial top view of CS and MV. (b) Schematic side view of $\mathrm{CS}$ and MV. . . . . . . . . . . . . . . . . 26

2.3 Three red lines connect the MV center to points $\mathrm{P}_{1}, \mathrm{P}_{2}$ and $\mathrm{P}_{3} \ldots \ldots 29$

2.4 (a) CS diameter at $\mathrm{P}_{3}$, (b) offset of CS from MA at $\mathrm{P}_{2} \ldots \ldots . . .29$

2.5 (a) Diameter of CS, (b) offset of CS from MA measured at three points of $\mathrm{P}_{1}, \mathrm{P}_{2}$ and $\mathrm{P}_{3} \ldots \ldots \ldots \ldots \ldots \ldots$

2.6 CS does not lie on the MP . . . . . . . . . . . . . . 31 
2.7 (a) Distance from CS to MA, (b) direct distance from CS to MA at three points of $\mathrm{P}_{1}, \mathrm{P}_{2}$ and $\mathrm{P}_{3} \ldots \ldots \ldots \ldots \ldots \ldots \ldots \ldots$

2.8 (a) Diameter of CS vs. gender, (b) Distance from CS to MA vs. gender 32

2.9 Correlations between CS parameters and LAV at different points of

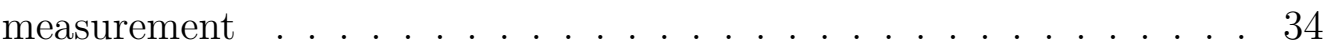

2.10 Experimental setup for measurement of applied force to MA from CS 37

2.11 Nonlinear behaviour of the MA under applied force because of the

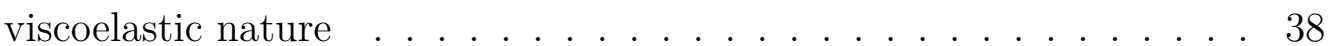

2.12 Sketch of the mechanism of applying force to MA with pushing arms 40

2.13 Mitral valve and CS . . . . . . . . . . . . . . . 42

2.14 Different lengths in the geometry of CS and MV . . . . . . . . . . 43

2.15 Different directions for describing the location of ERO . . . . . . . . 44

2.16 Atrial view of the final design for the catheter-based device . . . . . . 47

2.17 Posterior view of the final design for the catheter-based device showing the supports for tendons of releasing the one-directional joints . . 47

2.18 Anterior view of the final design for the catheter-based device showing the supports for actuation tendons . . . . . . . . . . . . . . . . 48

2.19 Ratchet mechanism for one-directional joints . . . . . . . . . . . . . . 48

2.20 Displacement of the link with ratchet one-directional joint . . . . . . 49

2.21 The pawl of the joint and tendon supports . . . . . . . . . . . . . . 52

2.22 The end part of a tendon and a sub-catheter of the steerable catheter 53

2.23 Grabbing and pulling a tendon by a sub-catheter of the steerable

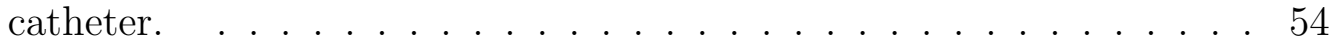

2.24 Female part of the engagement mechanism. Left: cross-sectional view. Right: Perspective view. . . . . . . . . . . . . . . 55 
2.25 Male part of the engagement mechanism. Left: top and side view. Right: Perspective view. . . . . . . . . . . . . . . 55

2.26 The target piece is guided to the target channel by the engagement mechanism.

2.27 Generated stress on the one-directional joint of the Link 1 while the link applies the force of $0.35 \mathrm{~N}$ to the MA . . . . . . . . 58

2.28 CFD simulation of blood inside CS. (a) Blood flow streamlines in CS without the implanted device. (b) Blood flow streamlines in CS with the implanted device. . . . . . . . . . . . . . . . . . . . . 59

2.29 CFD simulation of blood inside CS. (a) Velocity field of blood flow at $\mathrm{P}_{2}$ in CS without the implanted device. (b) Velocity field of blood flow at $\mathrm{P}_{2}$ in CS with the implanted device. . . . . . . . . . . 60

2.30 (a) Tendons supports and anchor, (b) Teeth of the ratchet joint, (c) Link and Pawl of the ratchet joint of the large-scale prototype of the device.

2.31 Large-scale prototype of the catheter-based tunable device for percutaneous treatment of MR . . . . . . . . . . . . . . 63

2.32 Experimental setup for validation of the designed engagement mechanism.

2.33 Camera is installed on the top of the experimental setup.

2.34 Different filters are applied on the picture captured by the camera to make it similar to X-ray image.

2.35 People tried to guide the catheter and engage two parts of the mechanism.

2.36 Procedure time versus trial number for ten people who performed the operation. 
2.37 Mean procedure time for each trial of the experiment of the engagement mechanism. . . . . . . . . . . . . . . . . . . 68

3.1 Sensing structure of the sensor, the front and rear parts of the structure. 71

3.2 FEM simulation results show the generated strain in the sensing structure due to the applied forces and torque to the structure. . . . . . . 74

3.3 Fabricated large-scaled prototype of the sensing structure with resistive strain gauges, (a) strain gauges number 1 to 4, (b) strain gauges number 5 to $8 . \ldots \ldots \ldots \ldots \ldots$

3.4 Experimental setup for applying lateral force to the large-scaled prototype of the sensing structure . . . . . . . . . . . . 76

3.5 Generated strains in the sensing structure under applied axial force, lateral force and torque. . . . . . . . . . . . . . . 77

3.6 Configuration of the strain gauges in the front part of the sensing structure. . . . . . . . . . . . . . . . . . . . . . . . .

3.7 Configuration of the strain gauges in the rear part of the sensing structure. . . . . . . . . . . . . . . . 80

3.8 FBG reflects a specific wavelength . . . . . . . . . . . . . . 85

3.9 Fabricated sensing structure . . . . . . . . . . . . . . 86

3.10 The assembled force/torque sensor that includes the sensing structure, optical fibers and catheter sheath. . . . . . . . . . . . . 87

3.11 Experimental set-ups for (a) applying axial forces and (b) applying lateral forces. . . . . . . . . . . . . . . . . . . . 89

3.12 Bragg wavelength shift of FBGs due to the axial force, lateral force

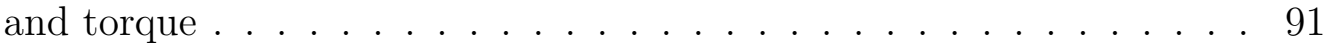

3.13 Experimental setup for applying torque. . . . . . . . . . . . . . 92

3.14 Experimental setup for applying dynamic force . . . . . . . . . . . . . 93 
3.15 Response of the fabricated sensor to the applied dynamic (a) axial force with sinusoidal wave and (b) lateral force with square wave. . . 94

3.16 Experimental setup for thermal sensitivity analysis of the sensor . . . 95

3.17 Bragg wavelength of FBGs and calculated forces by the sensor under applied axial force, lateral force and torque with temperature variation. 96

3.18 Measured applied forces/torque by fabricated sensor at different temperatures. . . . . . . . . . . . . . . . . . . . . 98 


\section{Nomenclature}

$\begin{array}{ll}\text { ABS } & \text { Acrylonitrile Butadiene Styrene } \\ \text { BSA } & \text { Body Surface Area } \\ \text { CS } & \text { Coronary Sinus } \\ \text { CT } & \text { Computed Tomography } \\ \text { ERO } & \text { Effective Regurgitant Orifice } \\ \text { EROA } & \text { Effective Regurgitant Orifice Area } \\ \text { EROSI } & \text { Effective Regurgitant Orifice Shape Index } \\ \text { FBG } & \text { Fiber Bragg Grating } \\ \text { FEM } & \text { Finite Element Method } \\ \text { GCV } & \text { Great Cardiac Vein } \\ \text { HDT } & \text { Left Atrium } \\ \text { HC } & \text { Interventional Cardiology } \\ & \\ & \end{array}$


Nomenclature

LCx Left Circumflex

LV Left Ventricle

LVDS Left Ventricular Diastolic Size

LVEF Left Ventricular Ejection Fraction

LVM Left Ventricular Mass

LVSS Left Ventricular Systolic Size

MA Mitral Annulus

MICS Minimally Invasive Cardiac Surgery

MR Mitral Regurgitation

MV Mitral Valve

SD Standard Deviation 


\section{Introduction}

\subsection{Interventional Cardiology}

Cardiac surgery is divided into two broad categories based on the method of operation: open cardiac surgery and minimally invasive cardiac surgery (MICS).

In open cardiac surgery, which is an invasive and conventional type of heart surgery, surgeons cut the chest and spread the rib cage to access the heart directly. On the other hand, in MICS, surgeons make small incisions in the skin, access the heart and great vessels through the incisions and perform the operation using special instruments $[1,2,3]$.

MICS, developed and applied since the mid-1990s, provides many advantages over open heart surgery technique including fewer postsurgical complications, short recovery time, less blood loss, lower risk of infection, less trauma and discomfort for patients [4].

Interventional Cardiology (IC) is a subclass of MICS in which surgeons insert catheters (thin flexible cylindrical devices) through main vessels (e.g. femoral vein) into the heart and perform the operation by translational and rotational movements of the catheter and also with controlling the knob which is embedded in the handle of the catheter (Figure 1.1). 


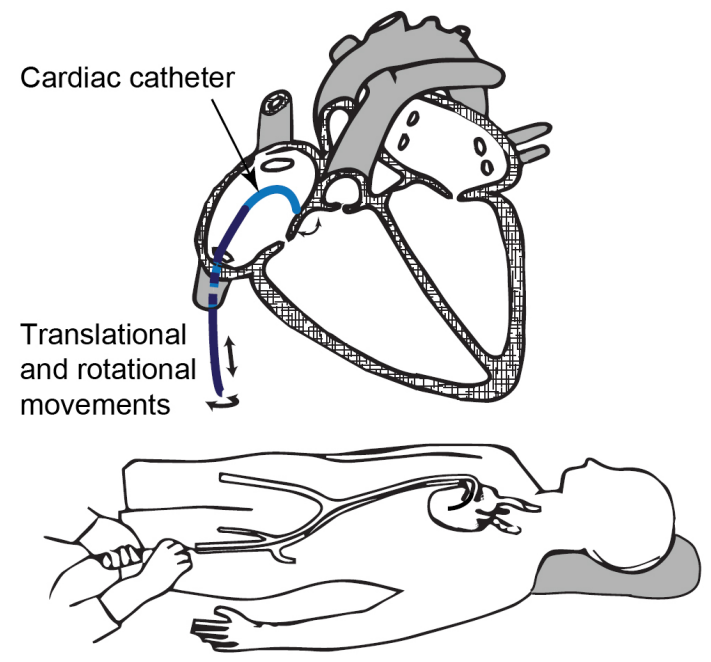

Figure 1.1: Interventional Cardiology

Despite many advantages of IC over open heart surgery, there is the main drawback of poor vision and tactile sense making IC a complicated and skill-based procedure for surgeons. Development of cardiac catheters and instruments with integrated sensors can help surgeons to perform IC easily and more accurately by providing beneficial information.

Another issue about IC procedures is tracking the catheter's distal end position during the surgery. The common method of catheter tracking during IC procedures is $\mathrm{X}$-ray imaging which is harmful to patients and surgeons, especially for the surgeons because they perform IC many times for different patients. Nowadays, there are some available surgical robots designed for IC surgeries to manipulate the catheter and carry out the operation by surgeons from a remote room such that the surgeon is not under X-ray radiation during the operation. Examples include Sensei Robotic Catheter System (Hansen Medical Inc., Mountain View, CA) and Amigo Remote Catheter System (Catheter Precision Inc., Ledgewood, NJ). Other advantages of surgical robots are higher accuracy and lower risk in comparison with manual IC procedures. Surgical robots need to get information from the surgery environment 


\subsection{Mitral Valve}

as the feedback to display for the surgeon or even to control the catheter and perform the IC automatically. Hence, development of sensors for cardiac catheters is vital for the evolution of surgical robots.

Development of robot-assisted procedures and automated surgical devices for IC has been the focus of the Robotics, Mechatronics and Manufacturing Automation Laboratory (RMAL). For instance, the Althea robot was developed in early 2000's in our laboratory (RMAL, Toronto, Canada). The ultimate objectives are to help interventional cardiologist to perform IC easily and accurately with reduces complications.

Among the cardiac problems, Mitral Regurgitation (MR) is the most common type of valvular disease affecting over 150 million people worldwide [5]. Therefore, the first objective of this thesis would be designing an IC device for MR treatment. Moreover, the second objective of the work is designing a force sensor for the safe delivering of the device which will also be useful for other cardiac catheters.

\subsection{Mitral Valve}

Mitral Valve (MV) is a valve inside of the heart which is located between the Left Atrium (LA) and Left Ventricle (LV) (Figure 1.2). It opens during diastole to let the blood flow goes from LA to LV and closes in ventricle systole preventing the blood flow from going back to LA. MV is a complex structure in the heart's anatomy with the components of the anterior mitral leaflet, posterior mitral leaflet, mitral valve annulus, the chordae tendineae and papillary muscles [6]. 




Figure 1.2: Mitral valve and its main parts

Mitral Annulus (MA) is a D-shaped fibrous ring that holds the anterior and posterior leaflets which are thin, pliable and soft textures. They are like veils with fixed edges, which are connected to the MA, and also with free edges. Free edges of the two leaflets coapt to close the valve. Posterior leaflet is divided into three scallops like sections: $\mathrm{P}_{1}, \mathrm{P}_{2}$, and $\mathrm{P}_{3}$. Anterior leaflet consists of three sections: $\mathrm{A}_{1}, \mathrm{~A}_{2}$, and $\mathrm{A}_{3}$. The chordae tendineae are thin fibrous strings that one end of them is connected to papillary muscles while the other end of it is attached to the leaflets. Papillary muscles move the leaflets with chordae tendineae so the mitral valve can open and close. Figure 1.3 shows the top view (atrial view) of MV.

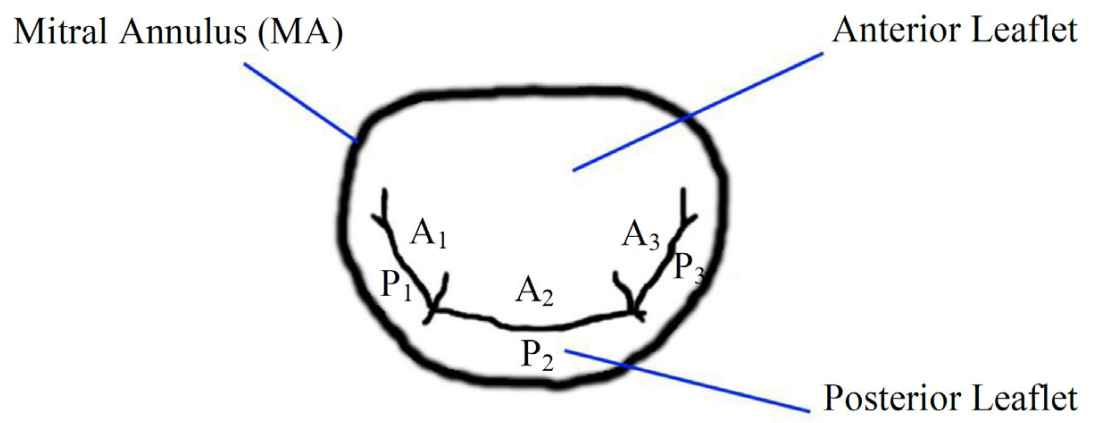

Figure 1.3: Atrial view of MV 


\subsection{Mitral Regurgitation}

Mitral Regurgitation (MR) is a heart disease related to the MV. In this disease, the MV does not close properly when the heart pumps blood to the body. This condition can happen due to dysfunctional performance of any of MV components. Indeed, in this disease, when the valve is in its closed position, there is a gap between anterior and posterior leaflets. MR can be divided into two categories: anatomic (or degenerative) MR and functional MR. In the former case there are abnormalities in the anatomy of mitral leaflets, papillary muscles, or chordae, while in the latter case changes in the size or geometry of LV or LA lead to dysfunction of MV [7].

\subsection{Treatment of MR by Interventional Cardiology}

During recent years, several devices have been introduced for percutaneous treatment of MR including MitralClip, Monarc, Carillon, St. Jude Medical device and Viacore, all explained in the following subsections. Among these devices, the first uses the Alfieri edge-to-edge technique and the rest work based on pushing MA through Coronary Sinus (CS). Because of the disadvantages of the first approach including unstable outcome and iatrogenic mitral valve stenosis, the second approach is more desirable.

\subsubsection{MitraClip}

With MitraClip device, one or several clips are utilized to bring the anterior and posterior leaflets together (Figure 1.4). The clips are delivered by a steerable catheter and placed at the location of maximum gap between the leaflets. By using this method and device, an incompetent single-orifice valve is converted into a competent 
double-orifice valve [8]. Clips are retrievable, and they can be repositioned to achieve the best outcome. This surgical method is based on the surgical Alfieri edge-to-edge technique and can be used for the treatment of both degenerative and functional MR [9]. In this technique, leaflets are sewed together from the middle part of the leaflets. Consequently, the gap between anterior and posterior leaflets decreases which results in decreasing MR [10]. Alfieri edge-to-edge technique is typically used with annuloplasty procedure where the surgeon places a ring around MV to reduce the gap between leaflets so they can coapt properly. If Alfieri edge-to-edge technique is not followed by an annuloplasty procedure, the results are usually suboptimal, and there is a significant probability of recurrent MR which results in a need for reoperation. Studies show that $30 \%$ of patients who had surgery using Alfieri edgeto-edge technique without annuloplasty need to have reoperation within five years because of recurrent $\mathrm{MR}$ while this percentage is $8 \%$ in those had Alfieri edge-to-edge technique with an annuloplasty procedure [11]. However, in MitraClip technology, annuloplasty is not used so the results are not optimal and there is a high probability of recurrent MR. This issue is the main drawback of MitraClip. Another limitation of MitraClip is that there is a possibility of causing iatrogenic mitral valve stenosis for this surgical method because the clips can restrict the mitral orifice and consequently cause mitral valve stenosis [12].

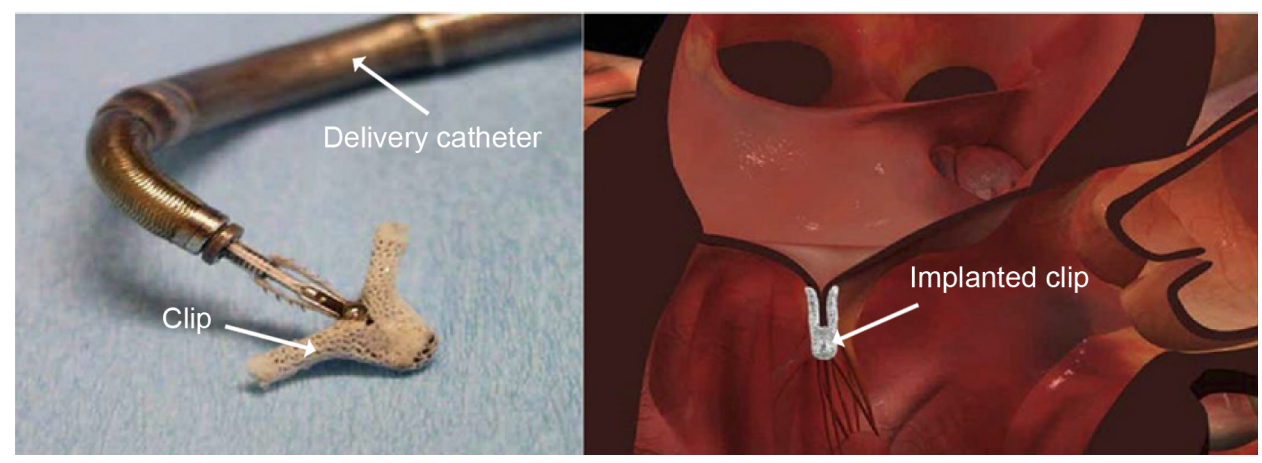

Figure 1.4: MitraClip system 


\subsubsection{Monarc}

In heart anatomy, there is a blood vessel which is called Coronary Sinus (CS) and is located around the heart for collecting deoxygenated blood from the heart muscles and delivering it to the right atrium. The location of CS is on the surface of the heart, and it is located almost in the mitral plane, the plane where MA is located. CS covers almost half of the MA as shown in Figure 1.5.

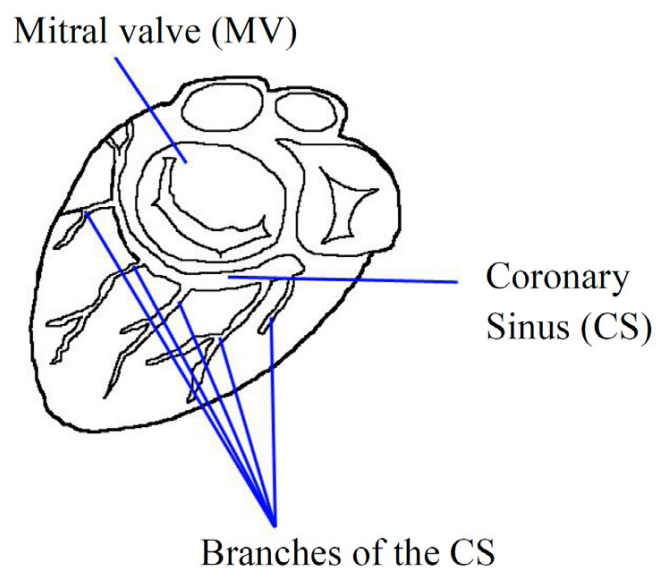

Figure 1.5: CS and it location with respect to MV

Monarc is a device which is designed based on the annuloplasty technique for MR treatment. In this approach, the device is delivered with a steerable delivery catheter and it is placed within the CS. This medical instrument consists of two anchors and a bridge which connects two anchors together (Figure 1.6). After deploying the distal anchor by retracting the outer sheath of the delivery catheter, the device is tensioned slightly to remove the gap between anterior and posterior leaflets. Then, the proximal anchor is deployed to hold the device inside of the CS. Indeed, because the CS is located almost in the mitral plane, the implanted device pushes the posterior part of MA anteriorly. Consequently, the diameter of the MA decreases which improves the leaflets coaptation and decreases the MR. There are two reasons for shrinking the MA with installing Monarc inside the CS. The first reason is tensioning of the 
device by the surgeon after deploying the first anchor and before deploying the second anchor (instant tensioning). The second reason is that the bridge between the two anchors of Monarc is like a spring with spacers which are made of biodegradable material. After implantation of the device, degradable material in the spring is absorbed over time and the bridge shortens (delayed tensioning).

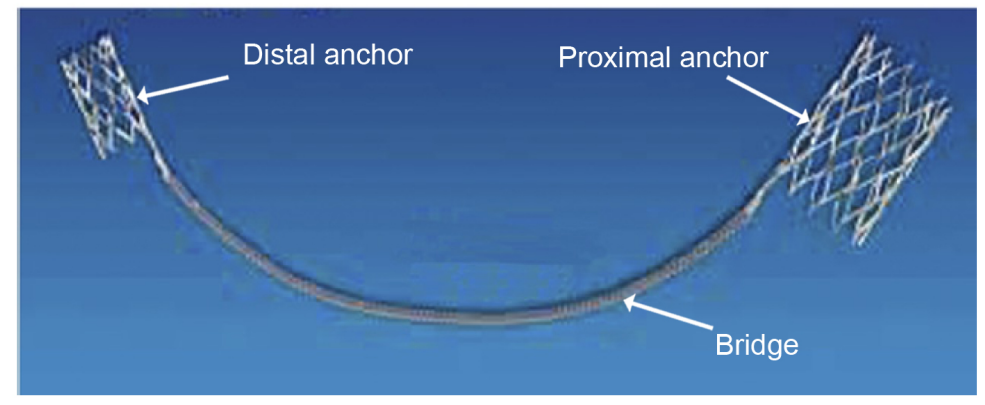

Figure 1.6: Monarc system

In a study, 5 patients with MR underwent implantation of Viking (first generation of Monarc). The device was implanted successfully in 4 patients, but the separation of the bridge happened in 3 of 4 implanted devices during 28 to 81 days [13]. In the next generation of this instrument, which is named Monarc, the bridge segment has been reinforced to avoid any separation of the bridge.

There is another issue for using this device for MR treatment arising from the shape, dimensions, and position of the CS which are not always appropriate for this approach of therapy. Coronary arteries branch off from Aorta artery, and are located on the surface of the heart for delivering oxygenated blood to the heart muscle. The two main coronary arteries are the right coronary artery and left coronary artery. The main branches of the left coronary artery are the left marginal artery (or obtuse marginal artery) and Left Circumflex (LCx) artery. There is a high probability that these branches of the left coronary artery are located under CS or great cardiac vein (GCV) which is part of CS. Therefore, if the implanted device pushes the posterior 
annulus anteriorly, these branches of the left coronary artery, which are located between CS and MA, will be compressed.

In a study that implantation of Monarc was investigated, the analysis of CS anatomy by Computed Tomography (CT) scan in 42 patients, who successfully received the implanted device, was performed. This study showed that CS or GCV pass over the LCx in 23 patients (55\%) and 8 of them (34.8\%) had angiographic coronary artery compression due to implantation of the Monarc. In contrast, in 19 patients the CS or GCV did not pass over a major branch of the left coronary artery, and none of them faced coronary compression after implantation of the Monarc [14]. This information reveals the main drawback of Monarc for MR treatment. In a different study, implantation of Monarc was unsuccessful in 18\% (13 out of 72) because of complicated shape or inappropriate dimensions of CS. Some degree of coronary artery compression happened in 15 patients of 50 patients who had the implanted device (30\%), and myocardial infarction occurred in 2 of them. From 50 patients, separation of the bridge from the proximal anchor happened in 4 cases (8\%) [14]. Another limitation of this device is that the CS might not be in proximity of the mitral plane so an effective annuloplasty might not take place.

\subsubsection{Carillon mitral contour system}

This device consists of two expandable anchors and a wire bridge which connects these two anchors together (Figure 1.7). In this instrument, tension is applied manually by the surgeon after deploying the distal anchor and before deploying the proximal anchor. Unlike Monarc, the bridge of this device is not made of a biodegradable material and there is no delayed tensioning effect [15]. The main drawback of this system is the probability of coronary arteries compressing after installing the device inside of the CS. Furthermore, CS might not be in proximity of the mitral plane. 
These two drawbacks were main drawbacks for Monarc as well because these two devices are similar and they have similar functions.

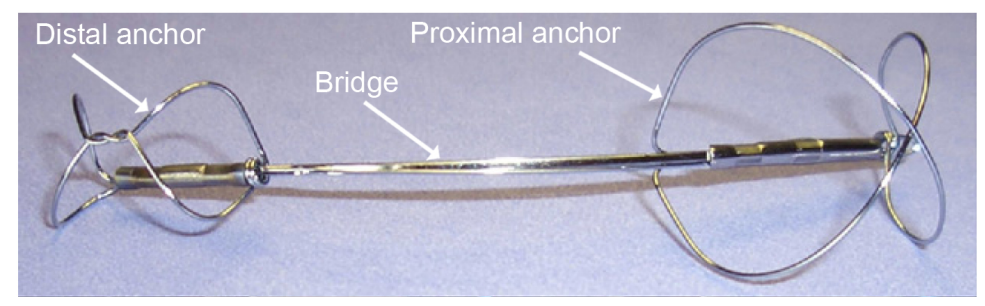

Figure 1.7: Carillon device

\subsubsection{Asymmetrical approach}

A device is introduced by St. Jude Medical (Minneapolis, Minnesota) which consist of four helical anchors, a bridge, and a delivery catheter (Figure 1.8). The two pairs of the anchors are connected by a bridge to shrink the MA. The implant system is delivered to the MA with endovascular techniques via the coronary sinus and right atrium using dedicated delivery catheters. Similar to Monarc and Carillon devices, this device utilizes the proximity of the CS to the MA and tries to shrink MA. However, unlike Monarc and Carillon, anchors of this device are not installed in $\mathrm{P}_{3}$ and $\mathrm{P}_{1}$ sections of the MV. The first pair of anchors for this device is installed in the $\mathrm{P}_{2}$ part of the MV while the second pair is installed out of the CS before its ostium and inside of the right atrium. Thus, the force which is applied to push the MA anteriorly is not symmetrical about the middle line of the section $\mathrm{P}_{2}$ of the posterior leaflet. There are some drawbacks for this device and technology including unknown long-term consequences of applying unequal tension on the CS or LA, possible risk of device fracture or erosion and also thrombus formation on the bridge. Also, there is a probability that some coronary arteries, which are located between CS and MA in the area that the device is installed, get compressed. 


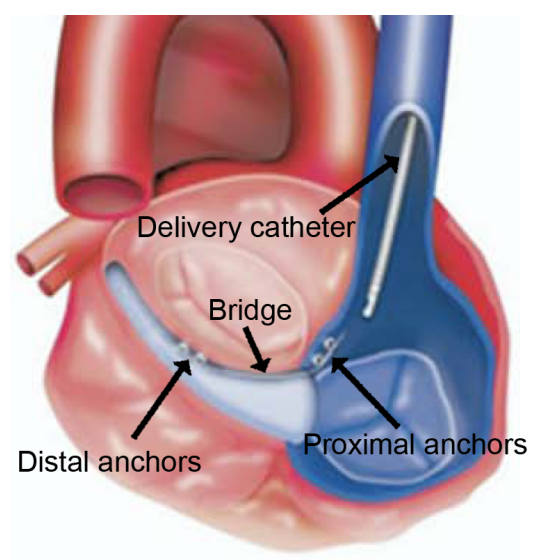

Figure 1.8: St. Jude Medical device

\subsubsection{Hollow tube with force applying structure}

This patented device consists of a hollow tube and a force applying structure attached to the tubular member (Figure 1.9) [16]. The force applying structure has a locking part, stop part and compression part, and it is fabricated from a stiff elastic biocompatible material such as stainless steel, cobalt-based alloys or biocompatible plastics. Applied axial force to the structure results in bending of the compression part. The stop element inhibits movement of the compression member's end when the axial force is applied. The function of locking part, which works based on the ratchet mechanism, is preventing the compression part from returning to its initial position. The compression part applies a force to push the MA anteriorly, so the diameter of the MA decreases. The force is applied to only one point of the MA, limiting the flexibility of treatment for patients. 


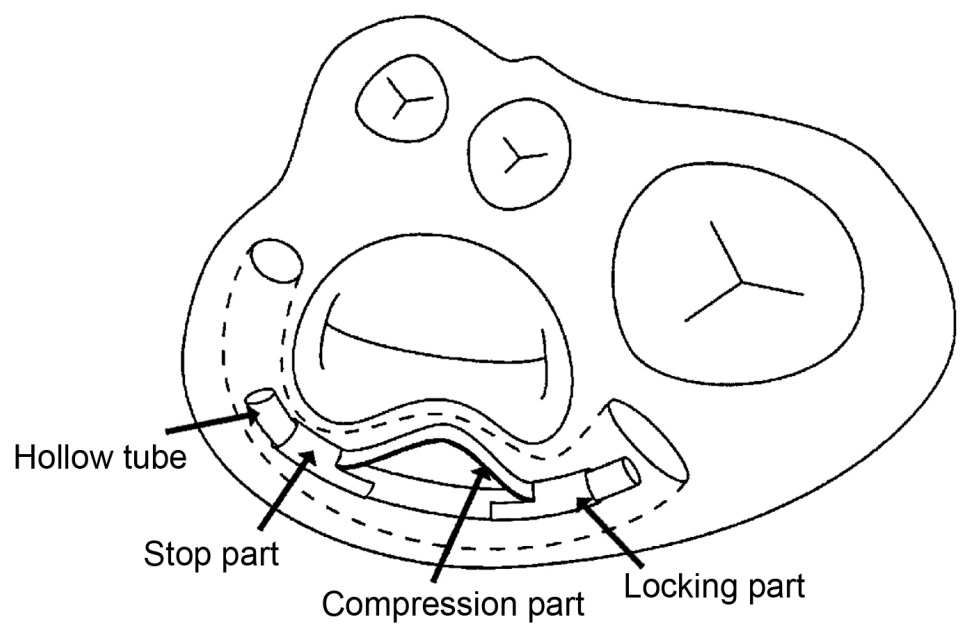

Figure 1.9: Hollow tube with force applying structure

\subsubsection{Viacor}

This device uses several rods with different length and stiffness. First, a hollow sheath is inserted into the CS and then the rods are inserted into the CS through the sheath. By retracting the sheath, the rods which were bent inside the sheath get straight and the force applied by the rods pushes the posterior leaflet towards the anterior leaflet; consequently, the gap between the leaflets decreases [17]. The main drawback of this instrument is the probability of coronary arteries compression due to the pressure that rods generate to push the MA anteriorly. Also, there is a possibility of fracture in the rods. Furthermore, after implantation of this device the shape of the MA will not be a D-shape ring and the rods will generate a flat line in the MA. The consequences of such a change in MA shape are unknown.

\subsubsection{Comparison of existing CS approaches}

The comparison between the existing devices in terms of mechanism of force applying and profile of the applied force are provided in the following subsections. 


\section{A. Mechanisms of force applying}

Monarc, Carillon, and the St. Jude Medical device utilize spring tension force to shrink the MA. The force in Viacor device is applied by the rods inserted into the CS. The device of the hollow tube with force applying structure applies the force to one point of MA to push the anterior leaflet towards the posterior leaflet. Table 1.1 lists the mechanism of force applying for the introduced devices.

Table 1.1: Mechanism of Force Applying in Different Devices

\begin{tabular}{|l|l|}
\hline Device name & Mechanism \\
\hline \hline Monarc & $\begin{array}{l}\text { spring tension force by } \\
\text { wire, instant and delayed } \\
\text { tensioning }\end{array}$ \\
\hline Carillon & $\begin{array}{l}\text { spring tension force by } \\
\text { wire, instant tensioning }\end{array}$ \\
\hline $\begin{array}{l}\text { Hollow tube with force } \\
\text { applying structure }\end{array}$ & $\begin{array}{l}\text { anterior force by the force } \\
\text { applying member }\end{array}$ \\
\hline St. Jude Medical device & spring tension force by wire \\
\hline Viacor & $\begin{array}{l}\text { spring tension force } \\
\text { generated by the rods }\end{array}$ \\
\hline
\end{tabular}

\section{B. Profile of the applied force}

For Monarc, Carillon, St. Jude Medical device and Viacor, the profile of the applied force along MA is uniform such that all parts of the MA experience almost the same amount of force. However, the device of the hollow tube with force applying structure can push the MA from a certain point resulting in a nonuniform profile of the applied force along the MA.

The comparison between the existing devices reveals that there is the lack of a catheter-based tunable device for percutaneous treatment of MR through CS that minimizes the compression of $L C x$. 


\subsection{Force sensor for cardiac catheters}

Measurement of magnitude and direction of the contact force between cardiac catheters's tip and body of the organ is very useful because it makes surgeons able to perform IC properly with high accuracy. For instance, in ablation surgery, if the catheter's tip has insufficient contact with the tissue of the heart, the generated lesions may not be effective enough to ablate the tissue. On the other hand, if the catheter's tip contacts the tissue with an excessive force, it can damage the tissue or even perforate the heart. Thus, the clinical result for ablation surgery highly depends on a proper contact between the catheter's tip and the heart tissue [18]. Getting the information on the magnitude and direction of the contact force helps the surgeon to make up for the lack of direct tactile and vision sense on the organ. Furthermore, there has been a trend towards performing IC by surgical robots in recent years. In this case, measurement of applied forces is vital for getting feedback and controlling the cardiac catheter. The surgical robot needs information about the contact force between the catheter's tip and the organ to guide and manipulate the catheter inside of the body or to display this information to the surgeon. Figure 1.10 shows the concept of applied axial and lateral forces to the tip of an ablation catheter.

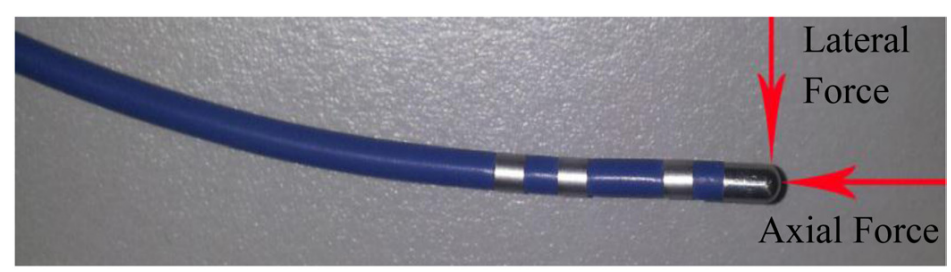

Figure 1.10: Axial and lateral forces applied to the tip of an ablation catheter

Bending in the distal end of cardiac catheters is generated mostly due to the lateral forces which are applied to the catheter's tip [19], therefore generated bending in the distal end of the catheters can be determined from the detected lateral applied 
forces to the catheter's tip.

Heart tissue does not have a smooth texture, so catheter's tip may be stuck in the heart tissue when the surgeon wants to rotate the catheter. Therefore, a torque can be generated in the distal end of the catheter. Measurement of this torque is important not only to get the feedback for robotic surgery but also to inhibit any injury to the heart's tissue. Hence, the torque should be measured as well as axial and lateral forces.

In the papers written by J. Rosen et al. and P. Kanagaratnam et al., a force/torque sensor was integrated in the instrument shaft outside the patient's body [20, 21]. Although this method provides some information of catheter-tip contact forces, however, frictional forces due to the interaction between the catheter's body and blood vessels disturb the measurement, so it cannot measure an accurate value of applied forces and torques to the distal end of the MIS instrument. It is noteworthy to mention that in cardiac catheters, the phenomenon of backlash aggravates the condition. Thus force/torque sensing of the instrument shaft, outside the patient body, cannot give an accurate amount of forces/torques of the instrument's distal end.

C. P. Tom introduced several methods to measure the magnitude and angle of the contact force between surgical instrument's tip and body tissue in a published patent [22]. The patent described several approaches including mechanical, capacitive, inductive, and resistive pressure sensing techniques. However, these types of pressure devices, which are very complicated in the structure, are vulnerable to electromagnetic interference. Moreover, these methods are temperature sensitive, causing errors in the measurement.

J. Peirs et al. in a paper and also N. Aeby and G. Leo in their patented design introduced a catheter having a tri-axial force sensor [23, 24]. A housing structure, which is embedded in the distal end of the catheter, functions as a force sensing 
structure. Three optical fibers, which are located inside the housing structure, measure the variation in the intensity of light reflected from the lateral surface of the reflector plate. The intensity of the reflected light changes due to the change in the distance between the fiber's tip and reflector plate which is caused by the applied force to the catheter's distal end. However, bending of the catheter and embedded optical fibers, causes a change in the intensity of the reflected light, generating an error in force reading of the catheter's distal end. Furthermore, temperature variation during surgeries (for instance ablation surgery), causes an error in the force measurement.

P. Polygerinos et al. introduced an MRI-compatible catheter which has the distal end force sensor [25]. When an axial or lateral force is applied to the distal end of the catheter, a flexible force-sensitive structure changes the distance and orientation of the reflective surface with reference to the tip of the embedded optical fiber. Although this sensor can detect the amount of applied axial and lateral forces, visual feedback from MRI-scanner should be used to distinguish between the axial and lateral forces. Furthermore, in this design, the optical fiber is placed in the center of the cardiac catheter, while in some medical instruments (e.g. cardiac catheters), the center of the instrument should be allocated for other elements like moving shaft or tendons. Other limitations of this sensor are being sensitive to temperature variation and not being sealed against body fluids (e.g. blood). After a few years, the same group (P. Polygerinos et al.) introduced a tri-axial force sensor embedded at the distal end of an ablation catheter [26]. A sensing structure deforms due to the applied forces to the catheter's distal end, and the intensity of the reflected light from a reflector's surface can be measured via three optical fibers. Consequently, the magnitude of the perpendicular and lateral forces can be measured. However, they used a polymeric material (Verowhite DM_8310-Gray 
25) for fabricating the sensing structure. Due to the Heat Distortion Temperature (HDT) of $41^{\circ} \mathrm{C}$ for this material, it is not proper for ablation surgery because the generated temperature by ablation catheter during the surgery reaches to $50^{\circ} \mathrm{C}$ and even more [27]. Also, this sensor is not sealed and body fluids could contaminate the inner sensing mechanism, resulting in a false measurement of applied forces. Furthermore, because of the light intensity modulation method of sensing in this sensor, an extra optical fiber has been placed in the center as a reference fiber to avoid the errors in the light intensity measurement due to bending. Moreover, this sensor cannot measure the torque generated in the distal end of the catheter. Being temperature sensitive is another drawback of this sensor.

S. J. Blumenkranz and D. Q. Larkin patented an idea about a force/torque sensor for cardiac catheters [28]. In this patent, groups of axially oriented strain gauges are embedded in the distal end of a catheter and are utilized to sense forces and torques at the distal end of the catheter. Although this sensor can measure lateral applied force without temperature sensitivity, temperature variation makes an error in the axial force measurement. Furthermore, this sensor cannot measure the the torque generated in the distal end of the catheter.

J. Arata et al. presented a temperature insensitive force sensor for medical applications based on a backbone shape structure [29]. Fiber Bragg Grating (FBG) has been used in this sensor for strain measurement. However, this sensor measures only the axial applied force.

I. Iordachita et al. introduced a temperature insensitive force sensor for the retinal microsurgery instrument. This sensor utilizes several FBGs for sensing purposes [30]. However, it measures the applied forces only in two directions in the plane perpendicular to the axis of the instrument.

A three-axis force sensor for cardiac catheters was introduced by Y. Noh et al. 
[31]. The sensor works based on the light-intensity modulation. The temperature variation in this sensor may cause displacements in the sensing structure and consequently produce some error in the reading. This sensor is not sealed, and its output can be affected by body fluid contamination. Moreover, this sensor cannot measure the the torque generated in the distal end of the catheter.

L. Xu et al. presented a temperature-insensitive contact force sensor for cardiac catheters was introduced [32]. The sensor works based on measurement of the total light power reflected by FBG embedded in the outer surface of the catheter. The catheter needs to be pre-bent so that the applied force to the tip of the catheter changes the curvature of the catheter. Indeed, this sensor is a bending sensor that measures the curvature of the catheter. Although this sensor may measure the applied force to the tip of the catheter without temperature cross-sensitivity, calibration of the sensor highly depends on the direction of the contact force. Therefore, the measurement error of this sensor in a real procedure will be higher than a controlled experiment in the lab. Furthermore, any generated curvature in the catheter due to other reasons rather than the contact force will cause an error in the readings. Based on the literature survey, there is no force sensor developed for cardiac catheters that can measure all components of the applied forces without thermal sensitivity.

\subsection{Research objectives}

The first objective of this proposed research is to develop a catheter-based tunable device for percutaneous treatment of MR through CS. The focus will be on designing a device which will enable the surgeon to correct the MR by changing the shape and diameter of the MA. This function of the instrument might be performed by applying forces to the MA from different points. Indeed, the applied forces by the 
device to the MA from different points of the CS will change the shape and also the diameter of the CS. This device should also be able to be adjusted and to modify the form and diameter of the MA after the first operation, which is done for delivering and installing the device into CS.

The device should be able to be delivered into CS by a steerable cardiac catheter and be installed inside of the CS. The delivery cardiac catheter may need a force sensor for delivering the device properly with a high accuracy. To guide the delivery catheter into the CS and also install the device inside of the CS, surgeon needs to track the device and the delivery catheter to make sure that the procedure goes properly. To do so, the surgeon can use X-ray imaging system, but there are some disadvantages to this method of tracking. X-ray imaging is harmful to both surgeons and patients. Furthermore, the surgeon does not get much information about contact force between the device and the body tissue. As this surgery is IC procedure and the surgeon does not have direct visual and tactile sense on the instrument, getting information about the contact force between the device and the body tissue helps the surgeon to perform the operation more accurately, so the risk of perforation of the body tissue by the device decreases. It also helps the surgeon to reduce the X-ray imaging time, so it decreases the side effects of imaging.

A force sensor will be an accessory device for the catheter-based device, and will help the surgeon to deliver and implant the device to the CS properly and more accurately. It is noteworthy to mention that the force sensor will also be useful for other applications in IC.

Force/torque sensors play a fundamental role in the development of surgical robots and smart cardiac catheters. However, the performed literature survey revealed that there is the lack of sensing assembly that can measure the force and torque applied to MIS instruments without temperature cross-sensitivity. Many designs 
were introduced to address this issue, but none of the proposed sensors can measure the all components of the applied force without temperature cross-sensitivity.

Therefore, the second objective of this research is to develop a temperature insensitive force sensor that can be used in cardiac catheters. In this project, cardiac ablation catheter is considered as a specific application of this sensor. Hence, dimensions of the sensor and its material were selected considering cardiac ablation catheters.

\subsection{Thesis contributions}

\subsubsection{Design of a catheter-based device for MR treatment}

The focus of this research project is designing a catheter-based tunable device which can be implanted inside the CS to modify the shape and diameter of the MA with the minimum compression of the LCx artery. The major problem for the current catheter-based devices, which are introduced based on CS approach for MR treatment, is compression of coronary arteries when the device is installed inside the CS. The design which will be presented in this research project addresses this drawback. The problem of the previously introduced devices is that the amount of applied force is uniform along the MA so if there is a coronary artery between the CS and MA, it will be compressed, resulting in fatal consequences. Between all these introduced devices, only one of them can apply non-uniform force to the MA. This device is a hollow tube with a force applying structure which is patented by N. Rafiee and et. al. in 2011 [16]. This device can apply force to one point of MA, limiting the surgeon to change the shape of MA without enough control.

The proposed design in this thesis enables surgeons to apply forces from different 
locations on the MA for modifying the shape of the MV. Also, if there is any coronary artery between the CS and MA, the surgeon will be able to avoid compressing it by applying forces from other proper points.

The design contribution involves the mechanical design using the geometric parameters of CS extracted from an extensive Computed Tomography (CT) scan analysis. Moreover, the proposed design implements safety measures for implanting inside CS. Various simulations and studies are performed to evaluate the safety of the proposed design.

The dimensional and spatial characteristics of CS are extracted by performing the comprehensive CT scan analysis of 204 patients images. The statistical analysis of the extracted data is presented and the correlations between the characteristics of the CS and physical/cardiac parameters of patients are studied. The obtained information from this analysis can be used to design the new IC surgical procedures.

\subsubsection{Force sensor for cardiac catheters}

In Section 1.5, several force sensors for cardiac catheters which are introduced by different research groups have been investigated. However, none of them can measure the all components of the applied forces without thermal sensitivity. Hence, another contribution of this research is designing a thermal insensitive 6DOF force/torque sensor for cardiac catheters.

\subsection{Thesis structure}

This thesis can be divided into two main parts: design of a catheter-based tunable device for percutaneous treatment of $\mathrm{MR}$ which is explained in Chapter 2, and 
1.8 Thesis structure

design of a temperature insensitive force/torque sensor for cardiac catheters which is presented in Chapter 3 of this thesis. Chapter 4 is the conclusion and future directions. 


\section{Catheter-based tunable device for percutaneous treatment of MR}

\subsection{Introduction}

Different devices developed for the percutaneous treatment of MR have been introduced in Section 1.4. The devices which are intended to be implanted inside CS and push the MA anteriorly suffer from two main drawbacks. The first issue is that there is no control on the amount of force applied to each $\mathrm{P}$ point $\left(\mathrm{P}_{1}, \mathrm{P}_{2}\right.$, and $\left.\mathrm{P}_{3}\right)$ of the posterior leaflet. The second issue is that there is a high probability of LCx artery compression for using such devices.

In this chapter of the thesis, a tunable catheter-based device that can be implanted inside CS and be adjusted by a steerable catheter is presented. The surgeon would be able to modify the shape of the MA and control the amount of applied force to each $\mathrm{P}$ point. The design of the device is such that the applied force on LCx artery is minimized.

The design of the device is presented in section 2.3 of this chapter. The obtained dimensional characteristics of CS which are crucial for designing the device are discussed in section 2.2.1 of this chapter as a subsection of section 2.2 (preliminary measurements). The safety evaluation of the design using simulations are presented in section 2.6 of this chapter. The section includes FEM simulation of the design under applied force for strength evaluation (section 2.6.1) and CFD simulation of blood flow inside CS for hemodynamic safety evaluation of the design (section 2.6.2). 


\subsection{Introduction}

It is noteworthy to mention that the amount of applied force from the device to MA, which is extracted through performing experiments and presented in section 2.2.2, is used in FEM simulation of the design under applied force. The large-scale prototyping of the design is presented in section 2.7 of this chapter. Moreover, the grabbing and pulling mechanism, and the engagement mechanism are presented in sections 2.4 and 2.5 of this chapter, respectively. The experimental validation of the engagement mechanism is provided in section 2.8 of this chapter. The diagram shown in Figure 2.1 shows how the items in this chapter are related to each other.

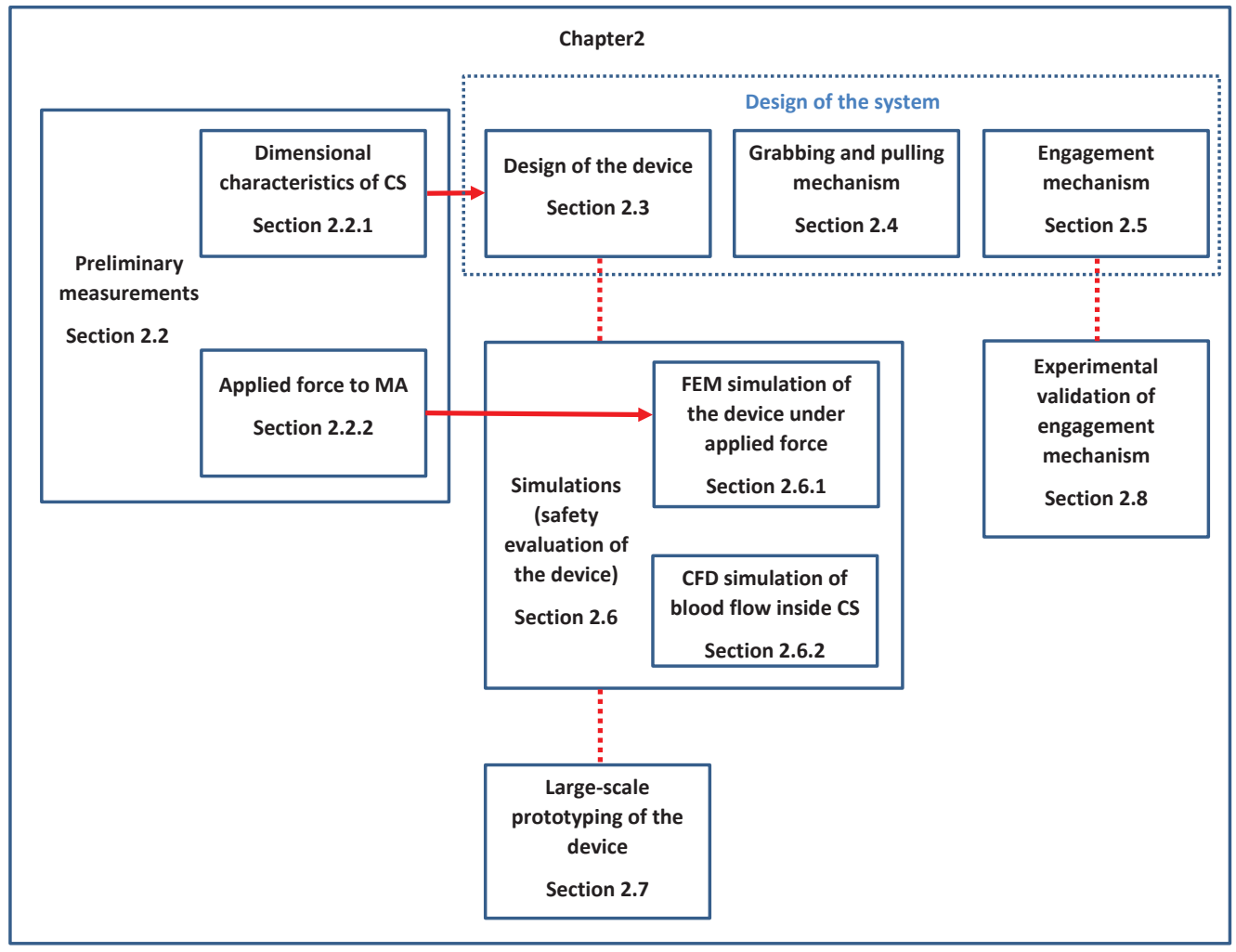

Figure 2.1: The relationship between different items of the chapter 


\subsection{Preliminary measurements}

For designing the device, some information on the size and geometry of CS should be considered. Literature survey in this area shows that there are some studies on dimensional and geometrical analysis of CS. However, these studies do not provide enough and complete information on CS size and its geometry at $\mathrm{P}$ points of the posterior leaflet. Therefore, in this research a comprehensive study is performed on dimension and geometry of CS with analyzing Computed Tomography (CT) scan images.

Another important parameter to be considered in designing the device is the amount of applied force at each $\mathrm{P}$ point required for pushing the MA anteriorly. The literature survey shows that there is no study done in this area. Thus, a set of experiments is performed in this research to extract the amount of force applied to the MA at $\mathrm{P}$ points. This section of the chapter provides the results of the related studies and experiments for obtaining this information.

\subsubsection{Dimensional characteristics of Coronary Sinus}

Figure 2.2 (a) shows the atrial view of the MV and CS. The parameter of the distance from CS to MA is presented in this figure. Figure 2.2 (b) shows the anterior crosssectional view of the MV and CS. This figure depicts all the location parameters of CS with respect to MA. 


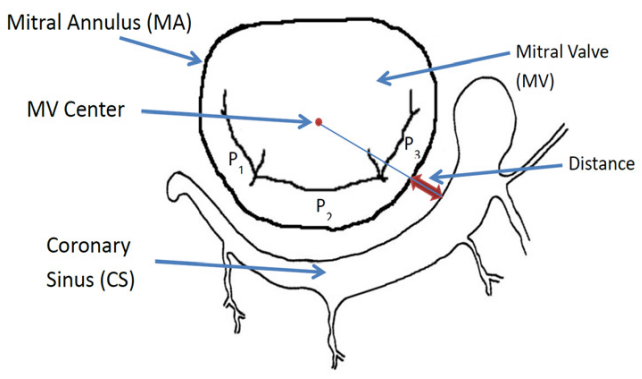

(a)

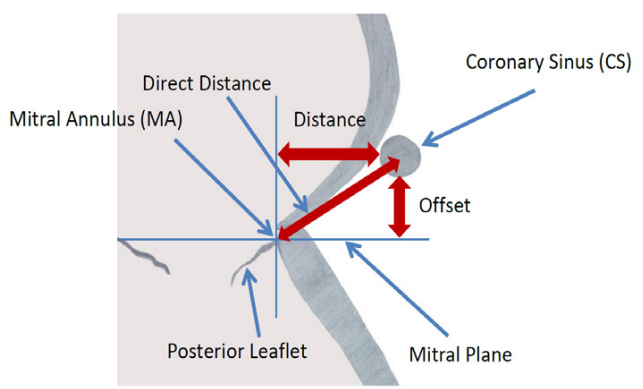

(b)

Figure 2.2: (a) Schematic atrial top view of CS and MV. (b) Schematic side view of CS and MV.

The value of the offset from CS to MA was studied by D. Maselli et al. at two points of $\mathrm{P}_{3}$ and $\mathrm{P}_{2}$. The reported values of this parameter were $9.7 \pm 3.2 \mathrm{~mm}$ and $5.7 \pm 3.3$ mm at $\mathrm{P}_{2}$ and $\mathrm{P}_{3}$ points, respectively [33], which shows an increase of offset (from CS to MA) from $\mathrm{P}_{3}$ point to $\mathrm{P}_{2}$ point.

Diameter of CS was studied by R. del Valle-Fernandez et al., and the pattern for this parameter was reported as a decreasing trend starting from CS ostium to the distal part of CS [34]. Diameter of CS was also studied by S. El-Maasarany et al.

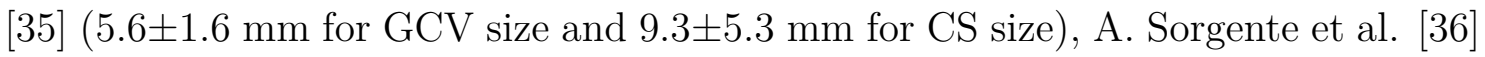
(11.78 $\mathrm{mm}$ for CS ostium diameter and $4.51 \mathrm{~mm}$ for GCV diameter), and D. Sahni et al. [37] (5.05 $\pm 0.97 \mathrm{~mm}$ at CS ostium, $4.32 \pm 1.0 \mathrm{~mm}$ at middle and $2.88 \pm 0.58 \mathrm{~mm}$ at distal end of CS-GCV) which all show a decreasing profile of CS diameter starting from CS ostium to its distal end. The reported profile of the CS-GCV diameter by A. Chiribiri also shows a decreasing pattern of this value starting from CS ostium to its distal end [38]. S. Mao et al. reported the diameter of CS only at CS ostium as $10.5 \pm 2.47 \mathrm{~mm}[39]$.

D. Valle-Fernandez et al. reported the value of the direct distance from CS to MA along the CS at every $10 \mathrm{~mm}$ starting from CS ostium [34]. The reported profile of the direct distance from CS to MA shows a maximum value at the middle point 
of the CS path. El-Maasarany et. al. reported the direct distance from CS to MA starting from CS ostium with steps of $36^{\circ}$. The reported profile shows the maximum point in the second region $\left(36^{\circ}-72^{\circ}\right)$ for $71.9 \%$ while for $28.1 \%$ of cases it is decreasing from CS ostium to the distal end of CS, continuously [35]. The direct distance from CS to MA was reported by J. S. Shinbane et. al. as $14.1 \pm 3.1 \mathrm{~mm}, 10.2 \pm 4.9 \mathrm{~mm}$ and $10.7 \pm 3.5 \mathrm{~mm}$ at 20, 40 and $60 \mathrm{~mm}$ from CS ostium, respectively [40].

The literature survey shows that the previous studies do not provide enough information on CS size and its geometry at $\mathrm{P}$ points of the posterior leaflet. Hence, the size of CS and its location with respect to the MA are studied in this thesis with analysis of CT scan images. The most critical factor for the function of the catheterbased MR treatment devices is the offset from CS to MA. The reason is that if the CS has a big offset from MA, the device cannot effectively push the posterior MA anteriorly to decrease the MR gap. Moreover, the dimensions of the catheter-based devices should be proper for the size of the CS.

In this study, CT scan data for 310 patients were investigated to extract the dimensions of CS and its location parameters with respect to MA. These patients underwent clinical assessment of presence or severity of coronary artery diseases at St. Michael's Hospital of Toronto. Out of this number of data, 204 of them had adequate properties for studying (74 females and 130 males, age $62 \pm 11$ ). The rest did not have enough resolution, or some parts of the CS or the MA were not captured in the images. The clinical characteristics of the study population are listed in Table 2.1. 
Table 2.1: Clinical characteristics of study population

\begin{tabular}{|l|c|}
\hline Characteristic & Value \\
\hline \hline Age, year, mean \pm SD & $62 \pm 11$ \\
\hline Gender, M/F & $130 / 74$ \\
\hline Weight, kg, mean \pm SD & $87.0 \pm 17.8$ \\
\hline Height, cm, mean \pm SD & $175.1 \pm 11.2$ \\
\hline BSA, ${ }^{2}$, mean \pm SD & $2.0 \pm 0.3$ \\
\hline Degree of MR & \\
\hline 0 & $159(78 \%)$ \\
\hline $1+$ & $38(19 \%)$ \\
\hline $2+$ & $7(3 \%)$ \\
\hline $3+$ & 0 \\
\hline $4+$ & 0 \\
\hline LAVI, ml $/ \mathrm{m}^{2}$, mean \pm SD & $35.1 \pm 9.8$ \\
\hline LVMI, g/m ${ }^{2}$, mean \pm SD & $86.3 \pm 20.0$ \\
\hline LVEF, $\%$, mean $\pm S D$ & $53.7 \pm 5.4$ \\
\hline Left Ventricular Diastole Size (Diameter), cm, mean \pm SD & $4.9 \pm 0.6$ \\
\hline Left Ventricular Systole Size (Diameter), cm, mean \pm SD & $3.2 \pm 0.6$ \\
\hline
\end{tabular}

The CT scan imaging process was performed with a 16-slice Toshiba Multislice Aquilion 0.5 (Toshiba Medical Systems, Otawara, Japan). Dimension and location parameters of CS that were measured are diameter of CS, offset of CS from MA, distance and direct distance from CS to MA. All measurements were performed at $75 \%$ of the RR interval. Measurements are performed in three points of $\mathrm{P}_{1}, \mathrm{P}_{2}$ and $\mathrm{P}_{3}$ (P points). These three points on the MA are related to three scallops $\left(\mathrm{P}_{1}\right.$, $\mathrm{P}_{2}$ and $\left.\mathrm{P}_{3}\right)$ of the posterior leaflet. The point $\mathrm{P}_{2}$ is located approximately in the middle of the $\mathrm{P}_{2}$ scallop. The angle between connecting lines from MV center to the $\mathrm{P}_{2}$ point and $\mathrm{P}_{1}$ point is around $60^{\circ}$; the angle between connecting lines from $\mathrm{MV}$ center to the $\mathrm{P}_{2}$ point and $\mathrm{P}_{3}$ point is also around $60^{\circ}$ [41].

For all of the measurements, the three points of $\mathrm{P}_{1}, \mathrm{P}_{2}$ and $\mathrm{P}_{3}$ are found and considered for measurement of CS size and location. Figure 2.3 depicts the three lines connecting the MV center to the points $\mathrm{P}_{1}, \mathrm{P}_{2}$ and $\mathrm{P}_{3}$ in a sample $\mathrm{CT}$ scan 
image. Measurements of CS parameters are performed at these three points by aligning a view plane on the respective connecting lines. For instance, Figure 2.4 (a) shows the measurement of CS diameter at $\mathrm{P}_{3}$ point which is $8.39 \mathrm{~mm}$ in this example. The measurements of CS offset and distance from MA are performed with the same method. Figure 2.4 (b) presents an example for measurement of CS offset from MA at $\mathrm{P}_{2}$ point which is $3.39 \mathrm{~mm}$. The 3D CT imaging software used for this analysis was Aquarius Software (TeraRecon Inc., San Mateo, CA) with the measurement accuracy of $0.1 \mathrm{~mm}$.

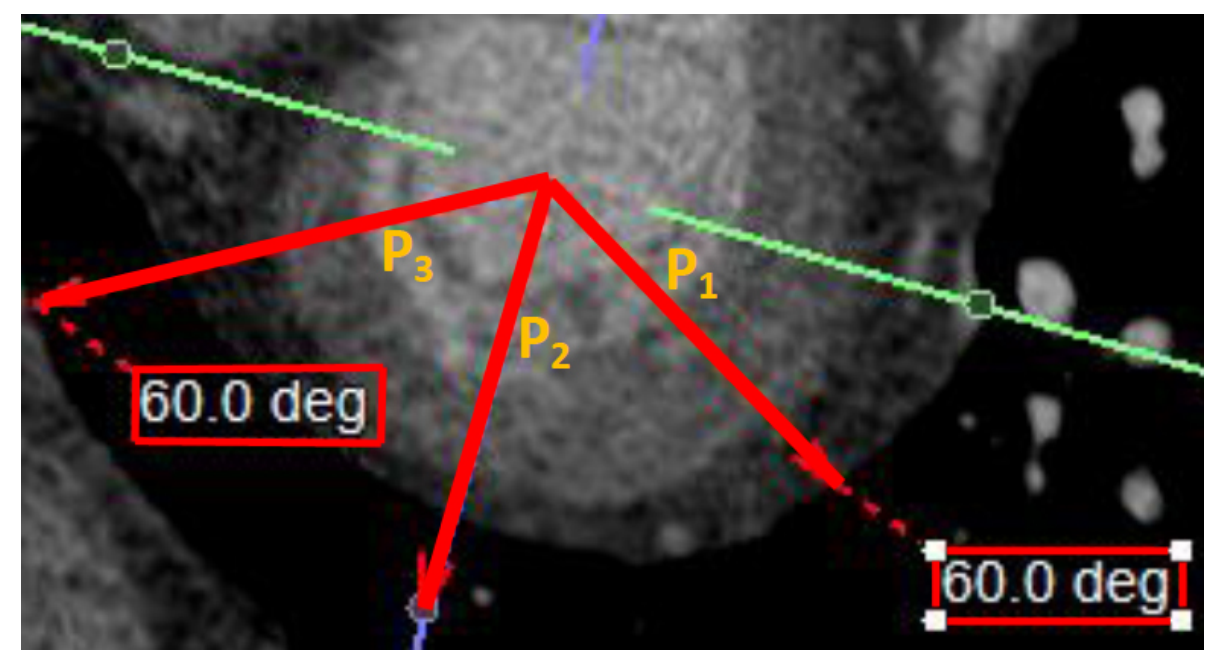

Figure 2.3: Three red lines connect the $M V$ center to points $\mathrm{P}_{1}, \mathrm{P}_{2}$ and $\mathrm{P}_{3}$

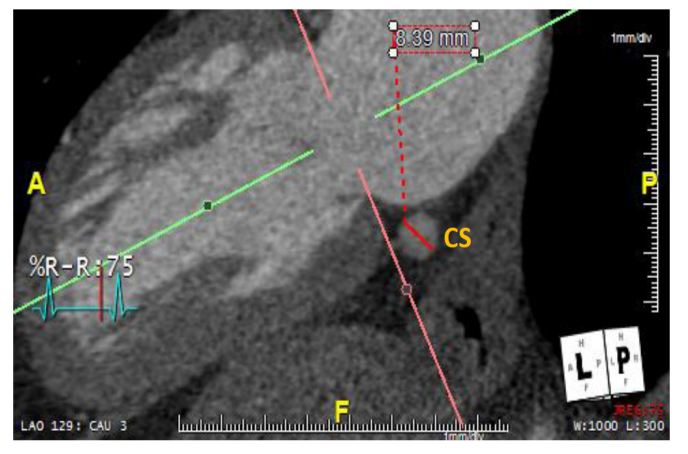

(a)

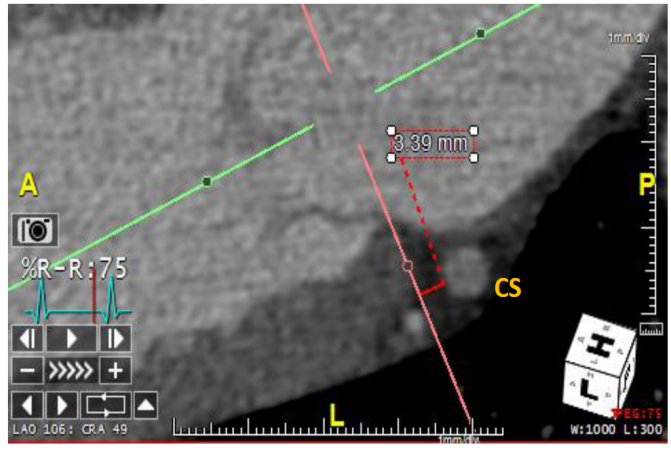

(b)

Figure 2.4: (a) CS diameter at $\mathrm{P}_{3}$, (b) offset of CS from MA at $\mathrm{P}_{2}$ 
In this study, the extracted continuous data are presented as mean values \pm Standard Deviation (SD) while the categorical data are provided as percentages and frequencies. IBM SPSS Statistics software (version 23, IBM Inc., New York) is used for performing all statistical analyses. Student's t-test is performed to find the difference between two sets of data. For finding the difference between three or more sets of data, the 1-way ANOVA test is performed. For these analyses, a $p$-value $<0.05$ has been considered statistically significant. The correlations ( $R$ values) in this study are computed with linear regression analysis. The following subsections present the results of the study.

\section{A. Diameter of CS}

The diameter of $\mathrm{CS}$ is $4.9 \pm 1.3 \mathrm{~mm}, 6.7 \pm 1.6 \mathrm{~mm}$ and $9.37 \pm 2.0$ at points $\mathrm{P}_{1}, \mathrm{P}_{2}$ and $\mathrm{P}_{3}$, respectively. The diagram of CS diameter at these three points is shown in Figure 2.5 (a). As shown in this figure, there is a significant decrease in diameter along the CS from $\mathrm{P}_{3}$ to $\mathrm{P}_{1}(\mathrm{P}<0.001)$.

\section{B. Offset of CS}

The offset of CS from MA is $4.7 \pm 2.6 \mathrm{~mm}, 8.8 \pm 3.4 \mathrm{~mm}$ and $5.4 \pm 3.3 \mathrm{~mm}$ at points $\mathrm{P}_{1}, \mathrm{P}_{2}$ and $\mathrm{P}_{3}$, respectively. Figure 2.5 (b) shows that this offset is maximum at $\mathrm{P}_{2}$ point among the three points $(\mathrm{P}<0.001)$. In Figure 2.6, the 3D generated image of a heart from CT scan data shows that the CS does not lie on the mitral plane and there is an offset from CS to MA. 


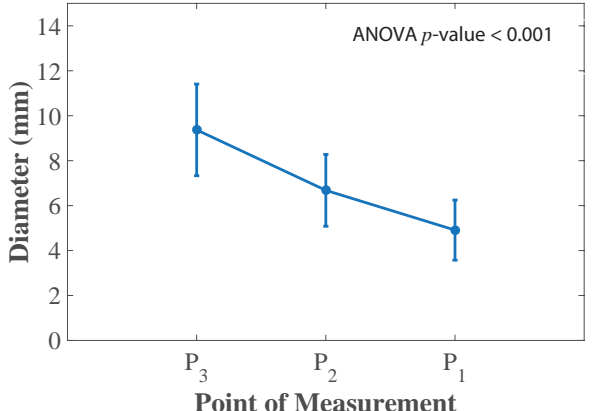

(a)

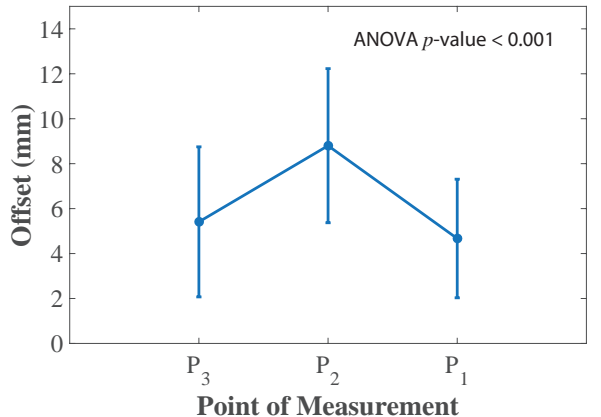

(b)

Figure 2.5: (a) Diameter of CS, (b) offset of CS from MA measured at three points of $\mathrm{P}_{1}, \mathrm{P}_{2}$ and $\mathrm{P}_{3}$

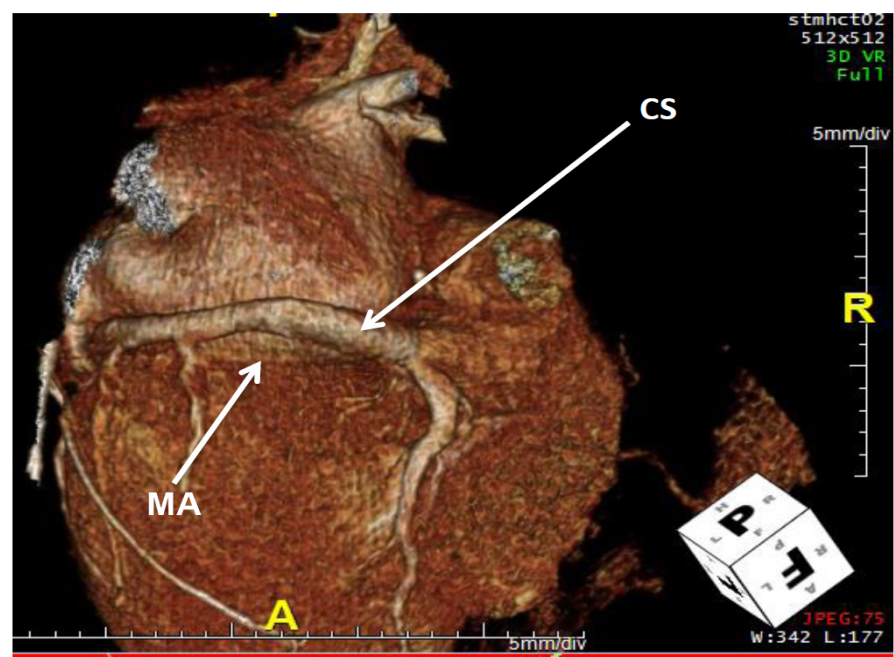

Figure 2.6: CS does not lie on the MP

\section{Distance and direct distance from CS to MA}

The distance from CS to MA is $5.5 \pm 3.2 \mathrm{~mm}, 3.6 \pm 2.8 \mathrm{~mm}$ and $4.5 \pm 2.7 \mathrm{~mm}$ at points $\mathrm{P}_{1}, \mathrm{P}_{2}$ and $\mathrm{P}_{3}$, respectively. The direct distance from CS to MA is $11.0 \pm 3.5 \mathrm{~mm}$, $14.3 \pm 3.5 \mathrm{~mm}$ and $13.9 \pm 3.2 \mathrm{~mm}$ at points $\mathrm{P}_{1}, \mathrm{P}_{2}$ and $\mathrm{P}_{3}$, respectively. Figure 2.7 depicts the profile for these two values from $\mathrm{P}_{3}$ to $\mathrm{P}_{1}$. 


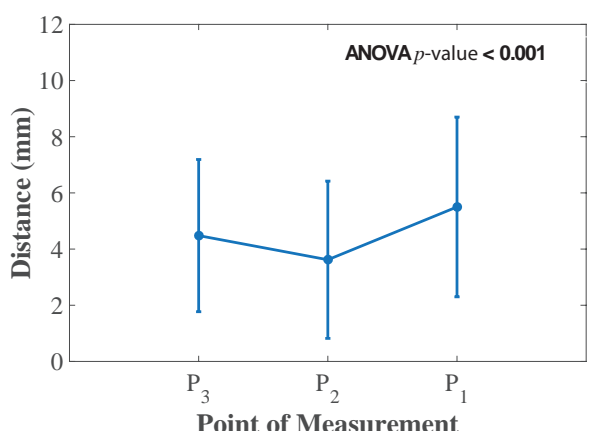

(a)

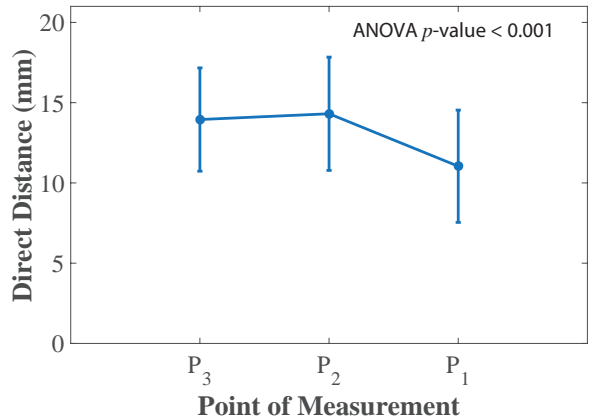

(b)

Figure 2.7: (a) Distance from CS to MA, (b) direct distance from CS to MA at three points of $\mathrm{P}_{1}, \mathrm{P}_{2}$ and $\mathrm{P}_{3}$

\section{Location and dimension of CS vs. gender}

The relationship between the parameters of CS and gender of the study population is investigated. Figure 2.8 (a) shows that the diameter of CS in female patients is significantly smaller than CS diameter in male patients. The distance from CS to MA is significantly less in male patients compared to female patients. This fact is shown in Figure 2.8 (b). There is no significant difference between the direct distance of CS to MA in male and female patients. There is also no significant difference between the offset of CS from MA in male and female patients.

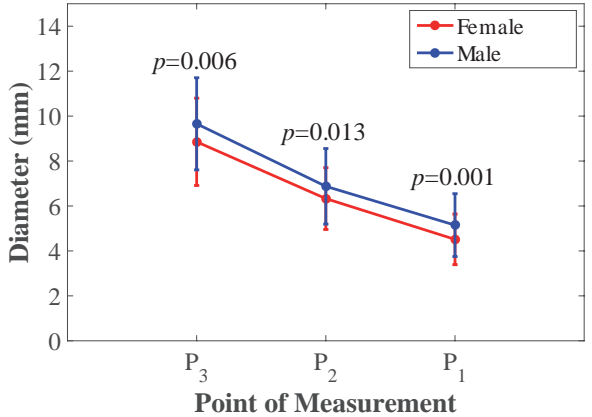

(a)

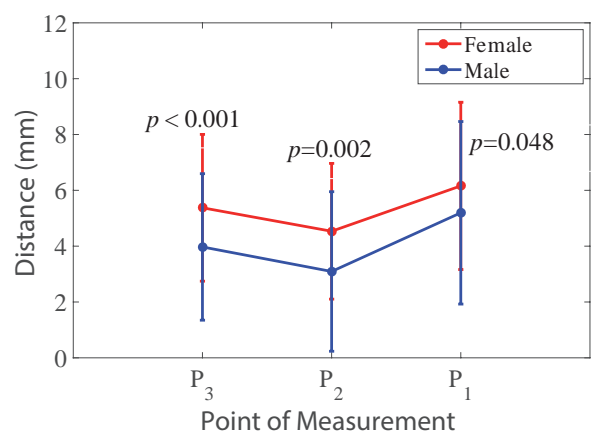

(b)

Figure 2.8: (a) Diameter of CS vs. gender, (b) Distance from CS to MA vs. gender 


\section{E. Correlation between diameter/location of CS and physical/cardiac parameters}

In this study, any possible correlation between the parameters of CS and physical/cardiac parameters is investigated. The considered physical/cardiac parameters are Body Surface Area (BSA), weight, height, age, MR grade, Left Atrial Volume (LAV), Left Ventricular Mass (LVM), Left Ventricular Ejection Fraction (LVEF), Left Ventricular Systolic Size (LVSS) and Left Ventricular Diastolic Size (LVDS). Correlations between these physical/cardiac parameters and diameter of CS at points $\mathrm{P}_{1}, \mathrm{P}_{2}$ and $\mathrm{P}_{3}$ have been investigated. A significant linear correlation between $\mathrm{CS}$ diameter and LAV was observed in points $\mathrm{P}_{1}(\mathrm{R}=0.33, \mathrm{P}<0.001), \mathrm{P}_{2}$ $(\mathrm{R}=0.45, \mathrm{P}<0.001)$ and $\mathrm{P}_{3}(\mathrm{R}=0.40, \mathrm{P}<0.001)$. There was no significant linear correlation between the diameter of CS and other studied physical/cardiac parameters. The parameter of LAV had a significant linear correlation with distance from CS to $\mathrm{MA}$ at $\mathrm{P}_{1}(\mathrm{R}=0.39, \mathrm{P}<0.001), \mathrm{P}_{2}(\mathrm{R}=0.28, \mathrm{P}<0.001)$ and $\mathrm{P}_{3}(\mathrm{R}=0.24, \mathrm{P}<0.001)$. There was also a significant linear correlation between LAV and direct distance from $C S$ to $M A$ in $\mathrm{P}_{1}(\mathrm{R}=0.47, \mathrm{P}<0.001), \mathrm{P}_{2}(\mathrm{R}=0.33, \mathrm{P}<0.001)$ and $\mathrm{P}_{3}(\mathrm{R}=0.53$, $\mathrm{P}<0.001)$. These correlations are shown in Figure 2.9. No other significant correlation between other physical/cardiac parameters and diameter/location of CS was observed. 


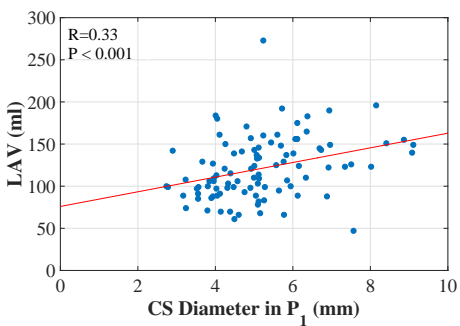

(a)

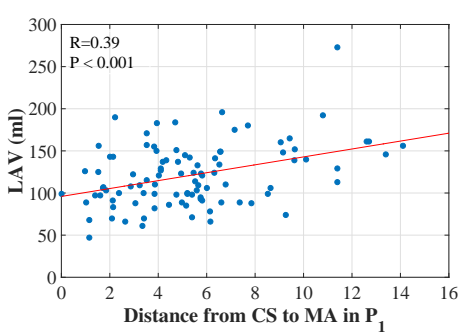

(d)

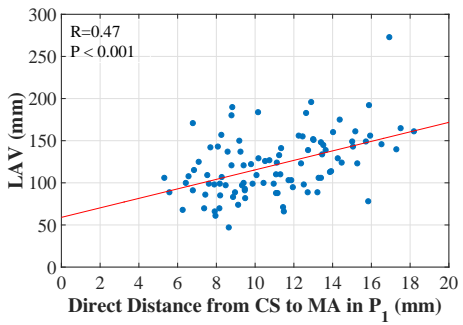

(g)

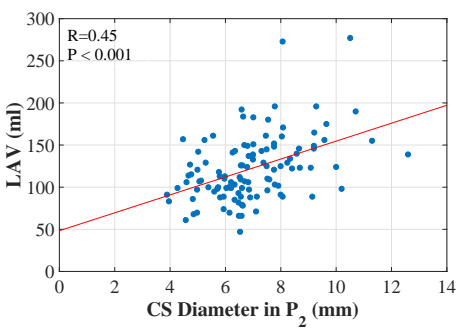

(b)

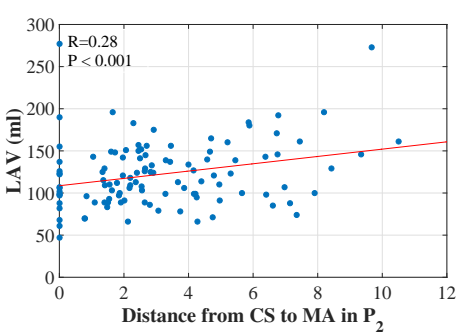

(e)

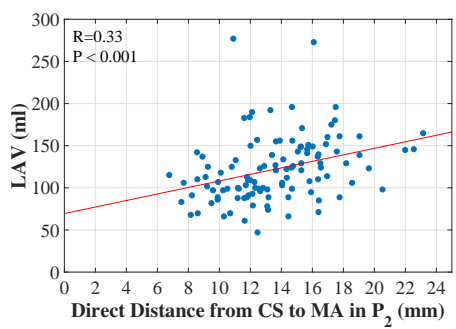

(h)

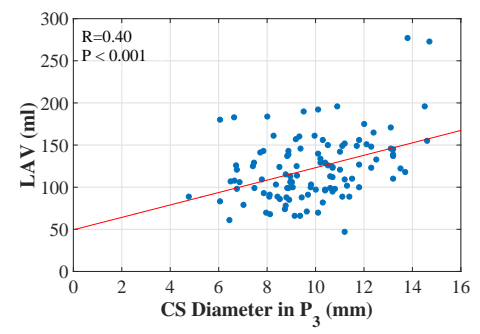

(c)

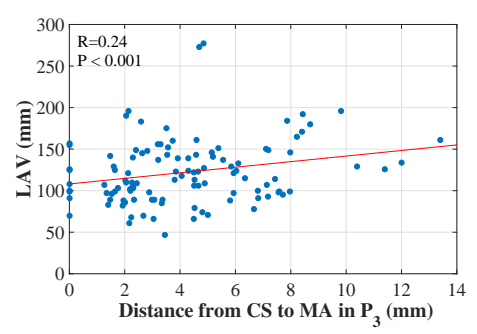

(f)

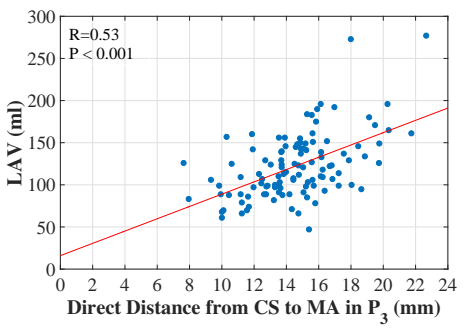

(i)

Figure 2.9: Correlations between CS parameters and LAV at different points of measurement

Table 2.2 shows the statistical results of the study for the diameter and location parameters of CS at three different points of $\mathrm{P}_{1}, \mathrm{P}_{2}$ and $\mathrm{P}_{3}$. 
Table 2.2: Statistical Results from Analyses of CS Diameter and its Location with Respect to MA

\begin{tabular}{|l|c|c|c|}
\hline & $\mathbf{P}_{\mathbf{3}}$ & $\mathbf{P}_{\mathbf{2}}$ & $\mathbf{P}_{\mathbf{1}}$ \\
\hline \hline CS diameter (mm) & $\begin{array}{c}9.37 \pm 2.04 \\
(4.77 \text { to } 14.7)\end{array}$ & $\begin{array}{c}6.68 \pm 1.60 \\
(3.26 \text { to } 12.6)\end{array}$ & $\begin{array}{c}4.91 \pm 1.34 \\
(2.03 \text { to } 9.11)\end{array}$ \\
\hline $\begin{array}{l}\text { Offset from CS to } \\
\text { MA (mm) }\end{array}$ & $\begin{array}{c}5.41 \pm 3.34 \\
(0 \text { to } 18.6)\end{array}$ & $\begin{array}{c}8.80 \pm 3.43 \\
(0 \text { to } 18.8)\end{array}$ & $\begin{array}{c}4.67 \pm 2.64 \\
(0 \text { to } 11.8)\end{array}$ \\
\hline Distance from CS to & $\begin{array}{c}4.48 \pm 2.71 \\
(0 \text { to } 15.1)\end{array}$ & $\begin{array}{c}3.62 \pm 2.80 \\
(0 \text { to } 12.9)\end{array}$ & $\begin{array}{c}5.50 \pm 3.20 \\
(0 \text { to } 18.8)\end{array}$ \\
MA (mm) & $\begin{array}{c}13.95 \pm 3.22 \\
(6.53 \text { to } 23.84)\end{array}$ & $\begin{array}{c}14.31 \pm 3.53 \\
(6.83 \text { to } 26.04)\end{array}$ & $\begin{array}{c}11.04 \pm 3.50 \\
(3.39 \text { to } 24.60)\end{array}$ \\
\hline CS to MA (mm)
\end{tabular}

The pattern of the offset from CS to MA (which has a maximum value at $\mathrm{P}_{2}$ point among the three important points of operation) is a major obstacle for using catheter-based MR treatment device inside CS. The device would not be effective enough due to this offset of CS from MA. However, the most important operation point for percutaneous MR treatment devices among the three measured points is $\mathrm{P}_{2}$. This point has the maximum gap in MR disease (both functional and degenerative MR) $[42,43]$. Thus, the offset of CS from MA at this point results in a major limitation for performance of the catheter-based devices.

\subsubsection{Applied force to the mitral plane and the generated displacement}

The extracted information from the CT scan analysis, which was presented in the previous section, is vital to design any device implanted inside the CS. The dimensional and spatial characteristics of the CS dictates the size of the designed device. However, these are other parameters to be considered in designing of the device. For instance, the amount of applied force from the device to the MA is a key factor 
in safety assessment of the device and should be considered in the design process.

The amount of force applied by the device to the MA should be in a proper range. A low amount of applied force will be ineffective for improving MR grade. On the other hand, an excessive amount of applied force can hurt or even puncture the heart tissue. In order to avoid such incident and to have a proper amount of the applied force, the proper range of the force that can be applied to MA from CS should be extracted. Furthermore, the amount of movement generated in MA due to the applied force needs to be found. Another reason for extracting the value of the required force is the evaluation of the device strength under the applied force using FEM simulations. The current subsection explains the performed experiment for measuring the required force applied to MA.

For performing the experiments, pig heart is used because of its structure, dimensions and function which are similar to the human heart [44]. The heart is suspended inside of a bucket of $\mathrm{NaCl}$ with using a stand during the experiment. Several sutures are utilized to suspend the heart from the stand. The temperature of the solution should be $37^{\circ} \mathrm{C}$ which is the usual temperature of the human body. A thermo circulating heater (Thermo Haake DC10) with a heating range of $25^{\circ} \mathrm{C}$ to $100^{\circ} \mathrm{C}$ is used for this purpose.

For temperature reading of the saline solution of $\mathrm{NaCl}$, Omegaette thermometer is utilized in this experiment. The resolution of this thermometer is $0.1^{\circ} \mathrm{C}$ and its accuracy is $\pm 1^{\circ} \mathrm{C}$. The range of temperature sensing for this thermometer is $-200^{\circ} \mathrm{C}$ to $1370^{\circ} \mathrm{C}$. The wire thermocouple of this thermometer is inserted into the bucket of $\mathrm{NaCl}$ solution to read the temperature of the solution. Based on the number which was shown by the thermometer, the temperature of the solution was $33.8^{\circ} \mathrm{C}$. This difference between the set temperature of the thermo circulating heater and the thermometer is because of the thermal loss from the heating source inside the thermo 
circulating heater to the heating element which is immersed in the $\mathrm{NaCl}$ solution. For increasing the temperature of the solution to almost $37^{\circ} \mathrm{C}$, the thermal circulator is set to $39.8^{\circ} \mathrm{C}$.

For measuring the amount of displacement of MA, a transparent ruler with 500 $\mu \mathrm{m}$ indicator is attached to the top of the heart with using a screw. The screw is inserted to the heart to keep the ruler exactly on the heart and on the MA to measure its displacement when the force is applied. ATI Nano-17 force sensor with the measurement resolution of $0.003 \mathrm{~N}$ is used to measure the force which is applied to the MA from CS location. For reading of the displacement from the ruler, which is attached to the heart, a desk magnifier with $7 \mathrm{x}$ magnification is used. A pump is used to pour the $\mathrm{NaCl}$ solution on the heart, so the portion of the heart which is outside of the $\mathrm{NaCl}$ is kept fresh during the experiment. Figure 2.10 shows the different parts of the total experimental setup.
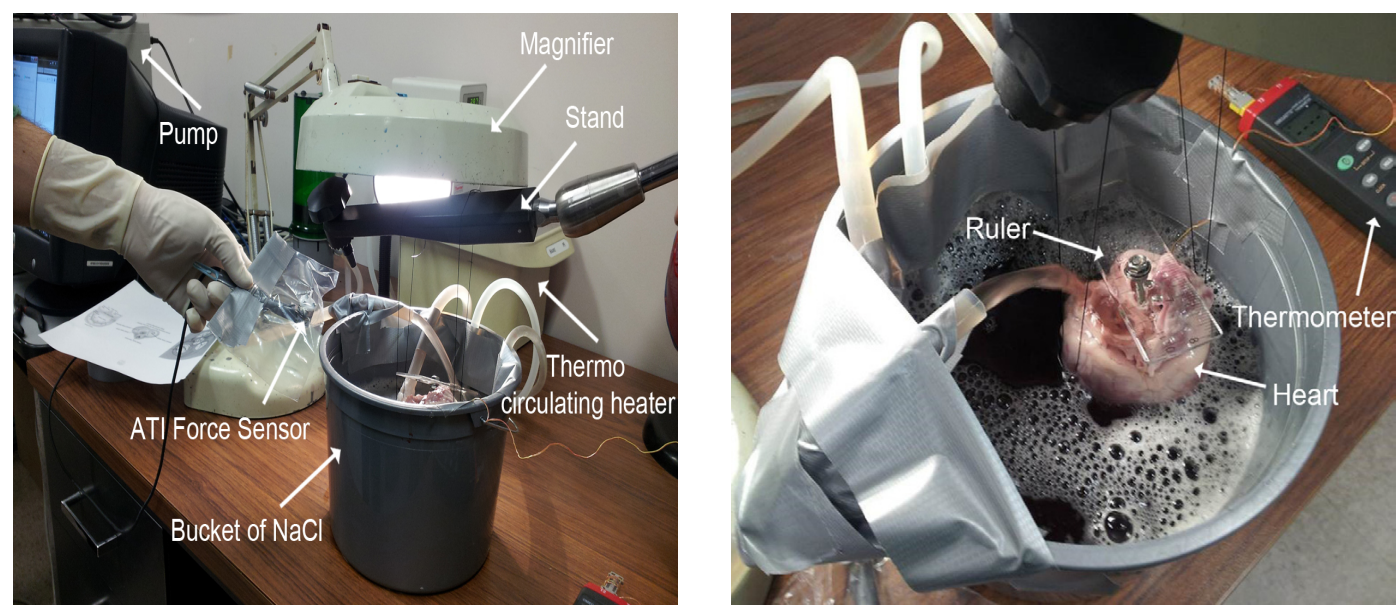

Figure 2.10: Experimental setup for measurement of applied force to MA from CS

The results of the experiment are presented in Table 2.3. 
Table 2.3: Applied Force for Generating Displacements at $\mathrm{P}_{1}, \mathrm{P}_{2}$ and $\mathrm{P}_{3}$

\begin{tabular}{|c|c|c|c|}
\hline Displacement & $\mathbf{1} \mathbf{~ m m}$ & $\mathbf{2} \mathbf{~ m m}$ & $\mathbf{3} \mathbf{m m}$ \\
\hline \hline Applied force at $\mathrm{P}_{1}$ & $0.075 \pm 0.009 \mathrm{~N}$ & $0.178 \pm 0.011 \mathrm{~N}$ & $0.298 \pm 0.012 \mathrm{~N}$ \\
\hline Applied force at $\mathrm{P}_{2}$ & $0.056 \pm 0.013 \mathrm{~N}$ & $0.103 \pm 0.014 \mathrm{~N}$ & $0.197 \pm 0.016 \mathrm{~N}$ \\
\hline Applied force at $\mathrm{P}_{3}$ & $0.080 \pm 0.011 \mathrm{~N}$ & $0.177 \pm 0.017 \mathrm{~N}$ & $0.327 \pm 0.019 \mathrm{~N}$ \\
\hline
\end{tabular}

The obtained results in this study for the amount of force applied to $\mathrm{P}_{2}$ point is in agreement with the results reported by M. O. Jensen et al. They showed in their study that the dynamic force applied to an annuloplasty ring at posterior point of the ring $\left(\mathrm{P}_{2}\right.$ point of $\left.\mathrm{MA}\right)$ is less than $0.4 \mathrm{~N}$ [45]. Moreover, the nonlinear behaviour of the MA as a soft tissue is shown in Figure 2.11. This behaviour of the MA is due to the viscoelastic nature of soft tissues.

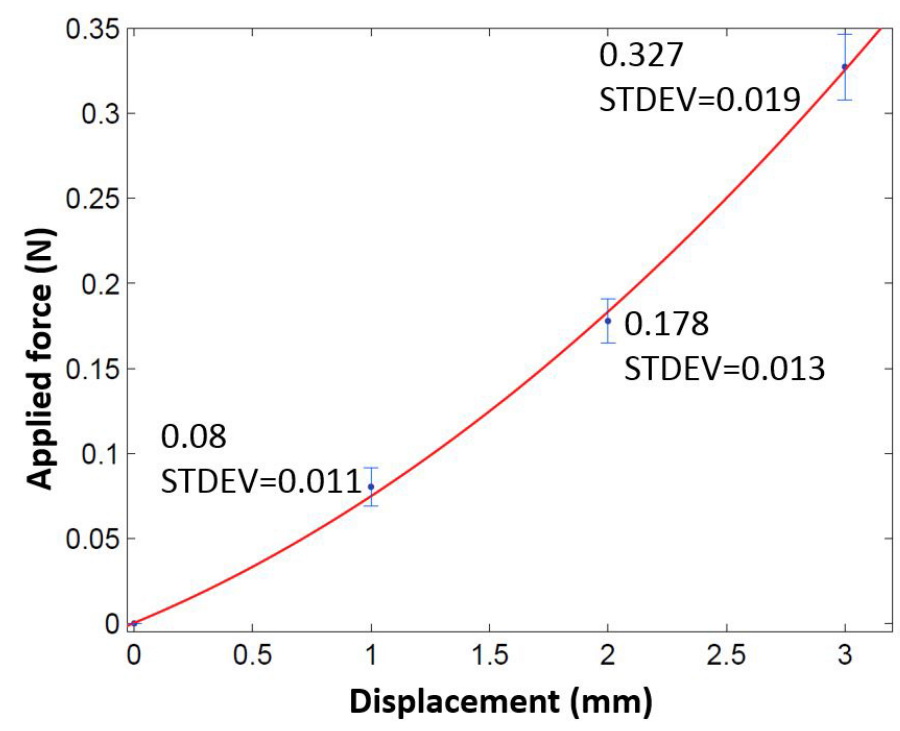

Figure 2.11: Nonlinear behaviour of the MA under applied force because of the viscoelastic nature

\subsection{Design}

The design procedure for the device is based on defining the design problem by providing the information on the product characteristics, functional requirements, and 


\subsection{Design}

constraints. For the catheter-based MR treatment device, the product characteristics can be listed as following: safe, deliverable and tunable by a steerable catheter. Safety is the most important characteristic that should be considered for this device. The material that will be used for fabrication of the device must be biocompatible. The device must be durable enough against breakage while applying force to MA. The device should be deliverable to the CS by a steerable catheter. Also, the position and the amount of applied forces by the device must be controllable by the surgeon.

The functional requirement for the device can be mentioned as the following statement. The device must apply forces to the MA and pushes it from different points anteriorly to decrease the gap between leaflets of the MV and improve the MR grade.

Structural constraints of the device is that it must be made from biocompatible materials. Also, dimensions of the device will be optimized with considering dimensions of the CS, so the device will be proper to be implanted inside CS.

Defining the design problem can be summarized as Table 2.4.

Table 2.4: Defining the design problem for desirable catheter-based device for MR treatment

\begin{tabular}{|c|c|c|c|}
\hline $\begin{array}{c}\text { Product } \\
\text { characteristics }\end{array}$ & $\begin{array}{c}\text { Functional } \\
\text { requirements }\end{array}$ & $\begin{array}{c}\text { Functional } \\
\text { constraints }\end{array}$ & $\begin{array}{c}\text { Structural } \\
\text { constraints }\end{array}$ \\
\hline \hline Safe & $\begin{array}{c}\text { Must decrease } \\
\text { the gap between } \\
\text { leaflets }\end{array}$ & $\begin{array}{c}\text { Generating } \\
\text { displacement of } \\
1 \text { to } 3 \mathrm{~mm}\end{array}$ & $\begin{array}{c}\text { Made from } \\
\text { biocompatible } \\
\text { material }\end{array}$ \\
\hline $\begin{array}{c}\text { MRI } \\
\text { Compatible }\end{array}$ & $\begin{array}{c}\text { must apply } \\
\text { forces to MA } \\
\text { from different } \\
\text { points }\end{array}$ & & $\begin{array}{c}\text { Dimensions of } \\
\text { the device }\end{array}$ \\
\hline $\begin{array}{c}\text { Controllable } \\
\text { steerable } \\
\text { catheter }\end{array}$ & & & \\
\hline
\end{tabular}




\subsection{Design}

The next step in the design process is forming the morphological chart as presented in Table 2.5. In this table, all possible options for different functions and parts of the device are considered.

Table 2.5: Morphological chart for design problem

\begin{tabular}{|c|c|c|c|c|}
\hline & Option 1 & Option 2 & Option 3 & Option 4 \\
\hline \hline $\begin{array}{c}\text { Implanted } \\
\text { inside CS }\end{array}$ & Stent anchors & $\begin{array}{c}\text { Implanted } \\
\text { anchors }\end{array}$ & Hollow tube & \\
\hline $\begin{array}{c}\text { Apply force } \\
\text { to MA }\end{array}$ & $\begin{array}{c}\text { Spring } \\
\text { between } \\
\text { anchors }\end{array}$ & Bending part & $\begin{array}{c}\text { Inflating } \\
\text { parts }\end{array}$ & Pushing arms \\
\hline $\begin{array}{c}\text { Power } \\
\text { source }\end{array}$ & $\begin{array}{c}\text { Surgeon } \\
\text { direct force }\end{array}$ & $\begin{array}{c}\text { Electrical } \\
\text { power }\end{array}$ & & \\
\hline $\begin{array}{c}\text { Feedback to } \\
\text { surgeon }\end{array}$ & x-ray imaging & Force sensor & & \\
\hline
\end{tabular}

Using the Pugh Concept Screening method, the final designed mechanism for the device based on the design procedure is pushing arms with an expandable stent anchor. A sketch of this mechanism is displayed in Figure 2.12.

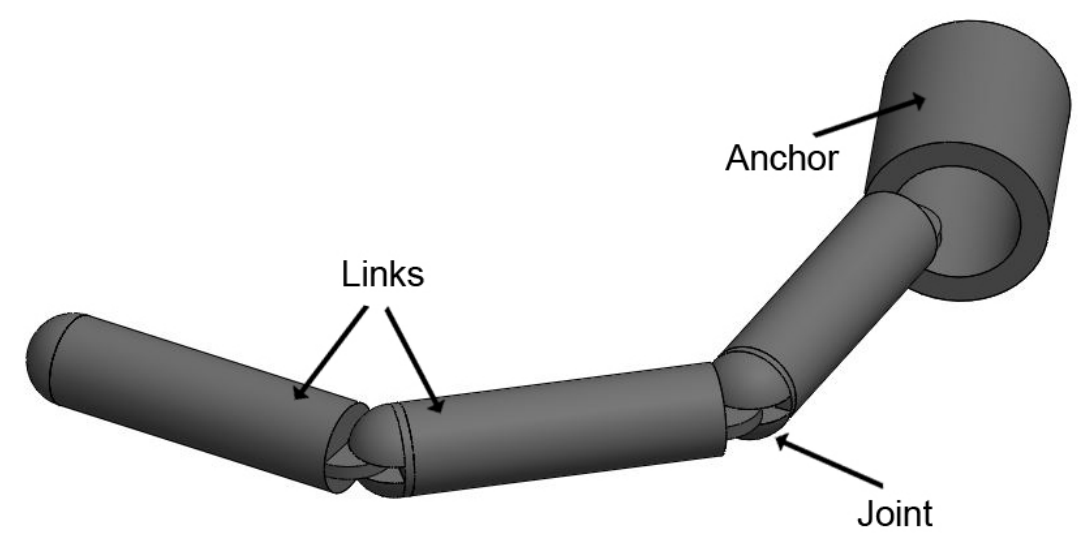

Figure 2.12: Sketch of the mechanism of applying force to MA with pushing arms

The shape of the links should be proper to minimize the safety risks. Thus, the links are designed with a circular cross-sectional shape. The anchor of the device can be 


\subsection{Design}

an expandable stent similar to Monarc design. For selecting the dimensions of the instrument and its links, dimensions and geometry of CS and MV were considered. CT scan images analysis and the previous studies are used to obtain the required information about the lengths between the P points along CS as explained below.

The angle between the lines crossing from the center of the MV and points of $\mathrm{P}_{1}$, $\mathrm{P}_{2}$ and $\mathrm{P}_{3}$ is approximately $60^{\circ}$ [41]. The radius of MA is approximately $15.5 \mathrm{~mm}$ [46]. It is noteworthy to mention that the diameter of MA varies during the cardiac cycle [34]. Here, the mean value of the measured radius in the end-systole and end-diastole has been selected with considering both genders of male and female.

The total length of CS-GCV which is almost on the mitral plane is $120 \mathrm{~mm}$, approximately $[34,36,47,48]$. The distance from CS ostium to the cross point between CS-GCV and LCx is reported as $79 \mathrm{~mm}$, approximately [38, 49].

The distance from the projection of CS ostium on MA to the posterior commissure point is approximately $3.6 \mathrm{~mm}$. This value is calculated from two measured distances: the distance from the projection of CS ostium to the right fibrous trigone which is $15.2 \mathrm{~mm}$, and the distance from posterior commissure point to the right fibrous trigone which is $11.6 \mathrm{~mm}$ [50]. Using these values, the distance from the projection of CS ostium to the $\mathrm{P}_{2}$ point on the MA is calculated as $27.5 \mathrm{~mm}$.

Figure 2.13 shows the MV and the CS from atrial top view with the geometrical parameters. The diameter of $\mathrm{CS}$ and its distance from MA at $\mathrm{P}_{1}, \mathrm{P}_{2}$ and $\mathrm{P}_{3}$ points are extracted from the performed CT scan images analysis. 


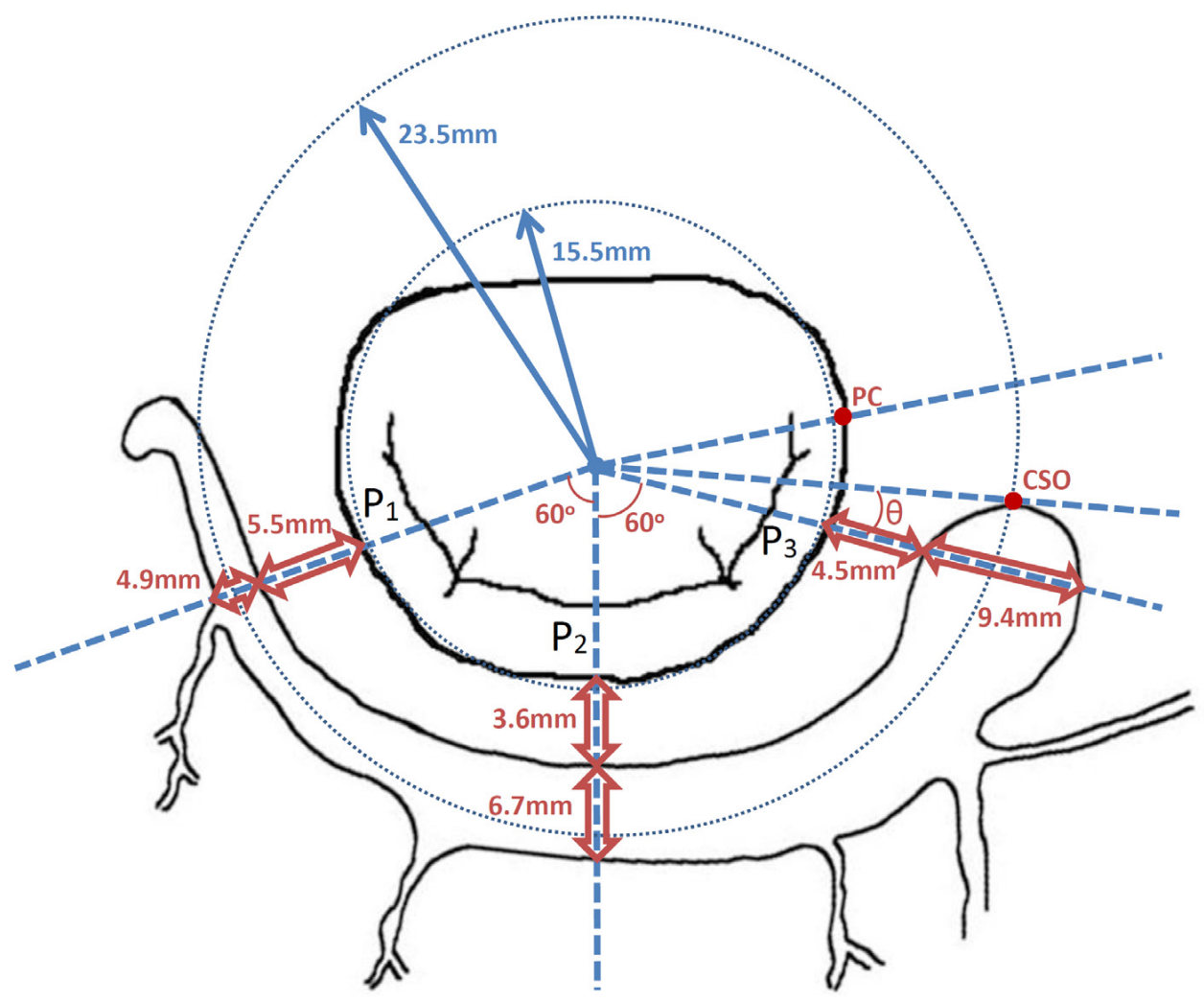

Figure 2.13: Mitral valve and CS

For calculating the lengths of the links for the device, two circles have been considered. As shown in Figure 2.13, the inner circle passes over the MA. Although MA does not have a circular shape and it is a D-shape structure, the assumed circle covers the circular part of the MA including $\mathrm{P}_{1}, \mathrm{P}_{2}$ and $\mathrm{P}_{3}$ points. The circumference of the circle on the MA is approximately $97.4 \mathrm{~mm}$. Thus, the angle $\vartheta$ is calculated as $41.6^{\circ}$.

From the calculations above, the length from the CS ostium to $\mathrm{P}_{3}$ point on the CS path $\left(\mathrm{L}_{1}\right)$ is $17 \mathrm{~mm}$, approximately. The length from the $\mathrm{P}_{3}$ point to $\mathrm{P}_{2}$ point $\left(\mathrm{L}_{2}\right)$ and from the $\mathrm{P}_{2}$ point to $\mathrm{P}_{1}$ point $\left(\mathrm{L}_{3}\right)$, both are $24.6 \mathrm{~mm}$, approximately. With considering the total length of the CS which is $120 \mathrm{~mm}$, the distance from the $\mathrm{P}_{1}$ point to the end of the CS-GCV $\left(\mathrm{L}_{4}\right)$ is approximately $53.8 \mathrm{~mm}$. The distance from 


\subsection{Design}

$\mathrm{P}_{1}$ point to the cross point between CS-GCV and $\mathrm{LCx}\left(\mathrm{L}_{5}\right)$ is calculated as 12.8 $\mathrm{mm}$. These lengths are shown in Figure 2.14.

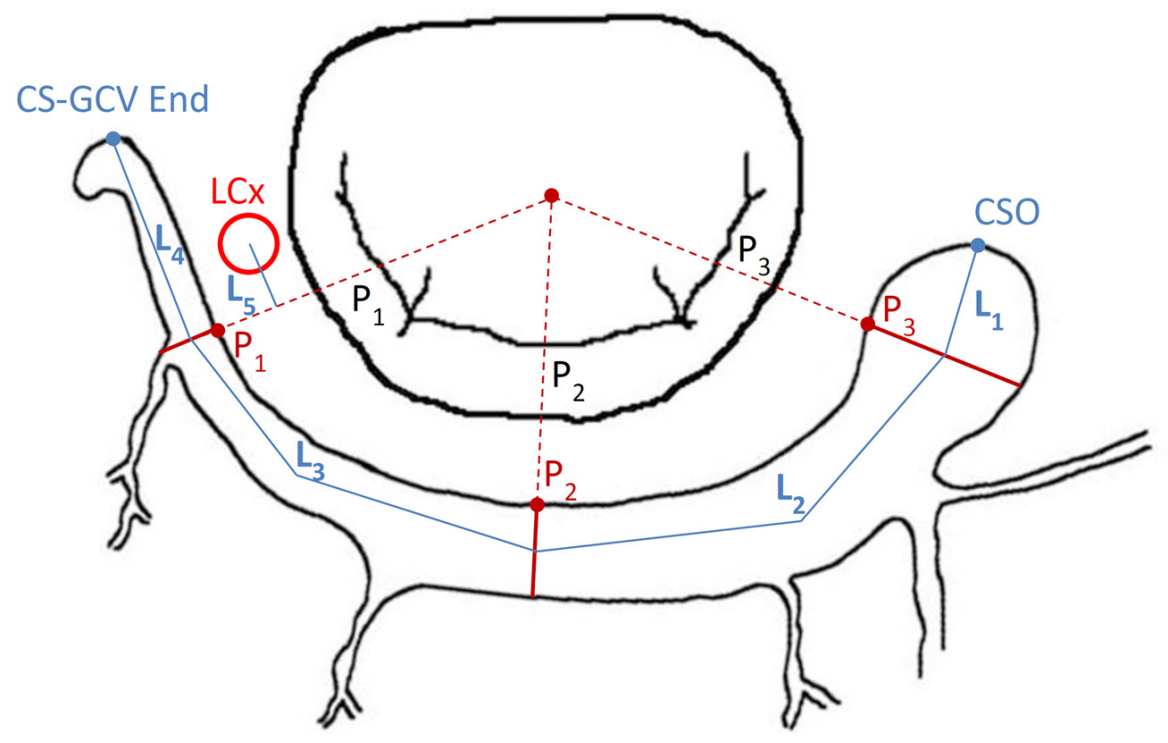

Figure 2.14: Different lengths in the geometry of CS and MV

There are two important questions that should be answered before finalizing the design of the catheter-based device. These questions are related to the gap between the leaflets of the MV which is generated in MR disease. The first question is about the location of the gap. Indeed, the answer to this question can help us in optimizing the final design to reach an efficient performance of the device. The second question is about the amount of gap between the leaflets of the MV in MR disease. The answer to this question is vital for designing the device because it will determine how much force should be applied by the device to decrease the gap. The answers of these questions are found based on the previous studies as explained below.

The orifice which exists in MV, when there is the MR disease, is called Effective Regurgitant Orifice (ERO) and the area of this orifice is called Effective Regurgitant Orifice Area (EROA). The severity of MR disease is determined based on the amount of EROA. MR disease is categorized in 4 grades (grade 1, 2, 3 and 4). Another 


\subsection{Design}

grading system categorizes the MR disease into mild, moderate and severe MR. EROA for mild, moderate and severe MR are less than $0.20 \mathrm{~cm}^{2}$, between 0.20 and $0.40 \mathrm{~cm}^{2}$ and more than $0.40 \mathrm{~cm}^{2}$, respectively [51].

The blood flow which passes the ERO in systole period of the cardiac cycle and enters the left atrium is called Mitral Regurgitant Jet which is usually analyzed for assessment of severity of the MR disease.

The location of ERO was investigated in previous studies. Some of these studies used the concept of Regurgitant Jet to determine the location of the respective ERO in the incompetent MV. In a study which was performed on 33 patients with MR disease, the direction of Regurgitant Jet was investigated [52]. The result of this study shows that in $66 \%$ of studied patients, the Regurgitant Jet direction was central while the rest of patients had posterior Jet direction. Although this study provides the information on the location of the ERO, this information is not very useful in designing of the percutaneous MR treatment device. The reason is that the location of ERO in that study is determined in the directions of anterior, central and posterior while the desirable directions for the location of ERO in the design procedure of the device are lateral, middle and proximal. Figure 2.15 shows all these directions.

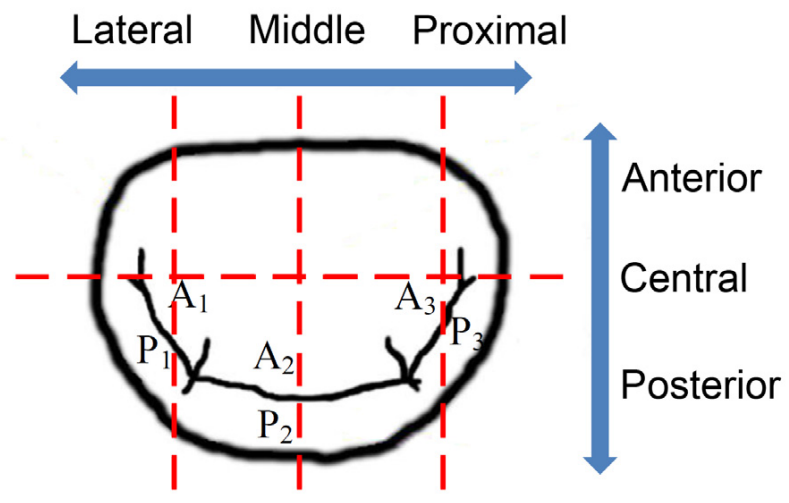

Figure 2.15: Different directions for describing the location of ERO 


\subsection{Design}

In a study, the shape of ERO and anatomy of MV apparatus were investigated in different types of MR [53]. The number of patients in the study was 74 . Out of this number, $39 \%, 27 \%$ and $34 \%$ of patients had mild, moderate and severe MR, respectively. This study included both types of functional and degenerative MR. Effective Regurgitant Orifice Shape Index (EROSI) was defined in this study as the ratio of the larger length $(L)$ to the smaller length $(W)$. In the elliptical shape of ERO, $L$ and $W$ are the length of major semi-axis and minor semi-axis, respectively. The results of this study showed that ERO has a significant asymmetrical shape, especially in the functional MR. The mean value of EROSI was reported as 3.91 and 1.24 in the functional and degenerative MR, respectively.

The elliptical shape of ERO is located in a way that the parameter of $W$ is in the direction of the long-axis view plane, approximately [42]. Indeed, applying force to the point of $\mathrm{P}_{2}$ will decrease the value of $W$ in the elliptical shape of ERO. This means that the most critical point between $\mathrm{P}_{1}, \mathrm{P}_{2}$ and $\mathrm{P}_{3}$ in functional $\mathrm{MR}$ is $\mathrm{P}_{2}$. In a different study, 68 patients with degenerative MR were investigated [43]. The location of leaflet prolapse was investigated and the results showed that the prolapse happens in the location of $\mathrm{A}_{1}$ or $\mathrm{P}_{1}$ in $7.3 \%, \mathrm{~A}_{2}$ or $\mathrm{P}_{2}$ in $36.7 \%, \mathrm{~A}_{3}$ or $\mathrm{P}_{3}$ in $17.6 \%$ of patients. The rest of patients had leaflet prolapse in multiple areas. This result reveal that $\mathrm{P}_{2}$ is the most critical point in degenerative MR as well.

If the shape of ERO is an ideal ellipse, the area of ERO can be calculated from $E R O A=\pi W L$, although this is an approximation of the actual value of EROA because the shape of ERO is not a perfect and ideal ellipse. The value of EROA in patients with functional MR (mild to severe) was measured in a study as $0.22 \pm 0.14$ $\mathrm{cm}^{2}$ [54]. With considering the mean value of 3.91 for EROSI in functional MR, which means $L / W=3.91$, the area of ERO can be written as $E R O A=\pi W L=$ $\pi W^{2} 3.91$. With considering the positive SD for the mean value of EROA, the 


\subsection{Design}

value of $W$ is calculated as $1.7 \mathrm{~mm}$. This amount of the gap between the leaflets of the incompetent $\mathrm{MV}$ is in the range of the generated displacement of $\mathrm{MA}$ in the performed experiment. Indeed, the the catheter-based device will be able to decrease the value of $W$ to zero.

In a study of 78 patients with at least moderate degenerative MR, the average value of the EROA was extracted as $43 \pm 20 \mathrm{~mm}^{2}$ [55]. With considering this mean value of EROA and the circular shape of ERO for degenerative MR, the value of diameter for ERO is calculated as $7.4 \mathrm{~mm}$. This value is larger than the maximum displacement that can be generated in MA. The reason is that the study did not consider patients with mild degenerative MR in the studied sample.

The atrial view for the final design of the catheter-based device for percutaneous treatment of MR is shown in Figure 2.16. The device consists of three links and an anchor. The links apply forces to the main three points $\mathrm{P}_{1}, \mathrm{P}_{2}$ and $\mathrm{P}_{3}$ of the MA. The lengths of the links are selected such that the location of the LCx is approximately placed close to the joint between Links 2 and 3. This way, the surgeon would be able to move the links such that the amount of applied force on the LCx is minimized.

The side view of the design for the device is shown in Figure 2.17. The diameters of the links are shown in this figure. As shown, the diameters of the links are decreasing from Link 1 to Link 3. This is based on the fact that the diameter of CS decreases along this artery. The maximum occupancy of the CS cross sectional area by Link 1, Link 2 and Link 3 are 42\%,36\% and 33\%, respectively. The total volume of CS/GCV is around $8528 \mathrm{~mm}^{3}$ which $814 \mathrm{~mm}^{3}$ of that $(9.5 \%)$ would be taken by the implanted device.

There are some supports considered in the design such that the tendons pass through them. The tendon supports in the anterior side of the device are placed in the design for actuation tendons. Three actuation tendons will be attached to the tip of the 


\subsection{Design}

links (one tendon for each link). Surgeons will be able to pull each tendon and change the position of each link. The joints of this device are designed as one-directional so that the links can stay in the desired position and not pushed back by the MV. The longitudinal cross-sectional view of the device shown in Figure 2.19, depicts the ratchet mechanism of one-directional joints of the device.

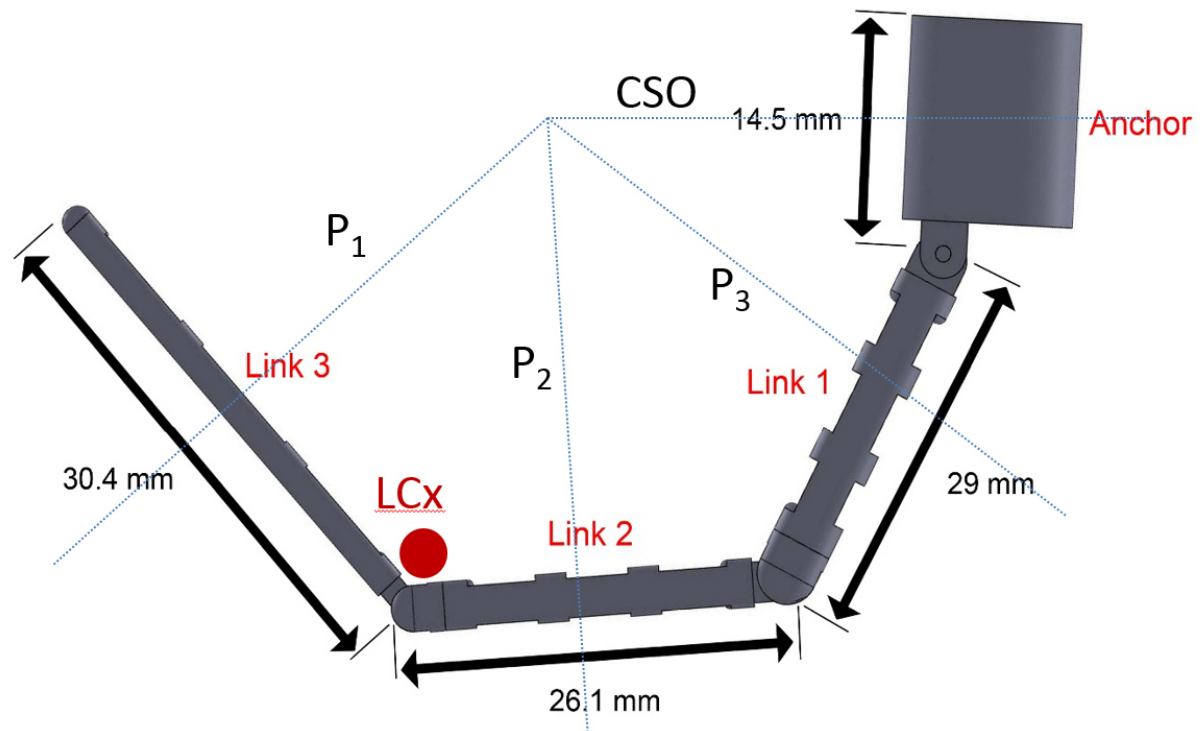

Figure 2.16: Atrial view of the final design for the catheter-based device

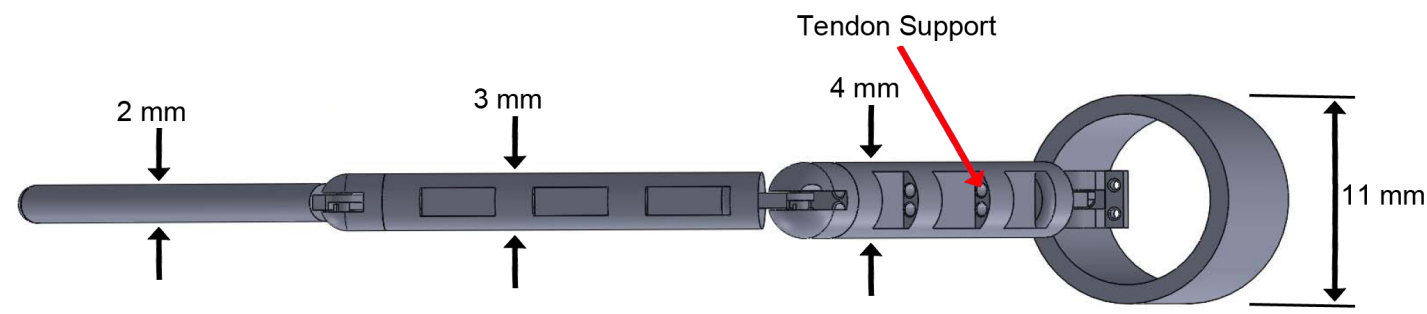

Figure 2.17: Posterior view of the final design for the catheter-based device showing the supports for tendons of releasing the one-directional joints 


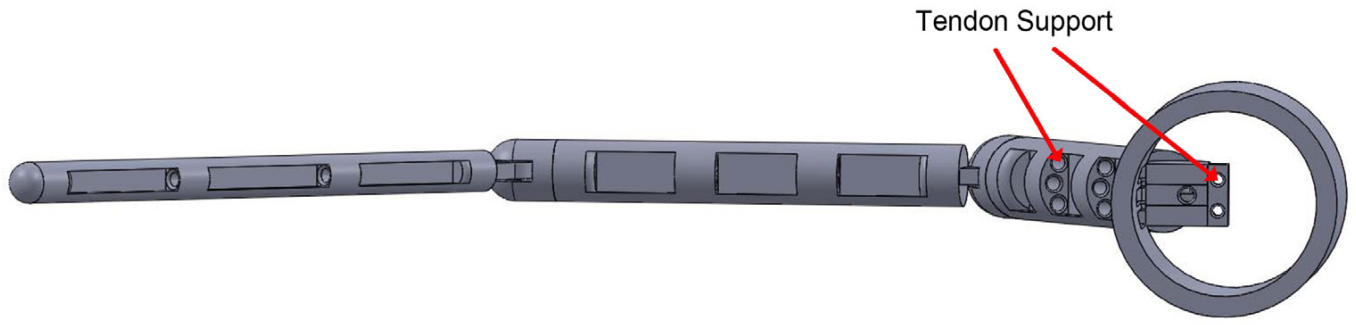

Figure 2.18: Anterior view of the final design for the catheter-based device showing the supports for actuation tendons

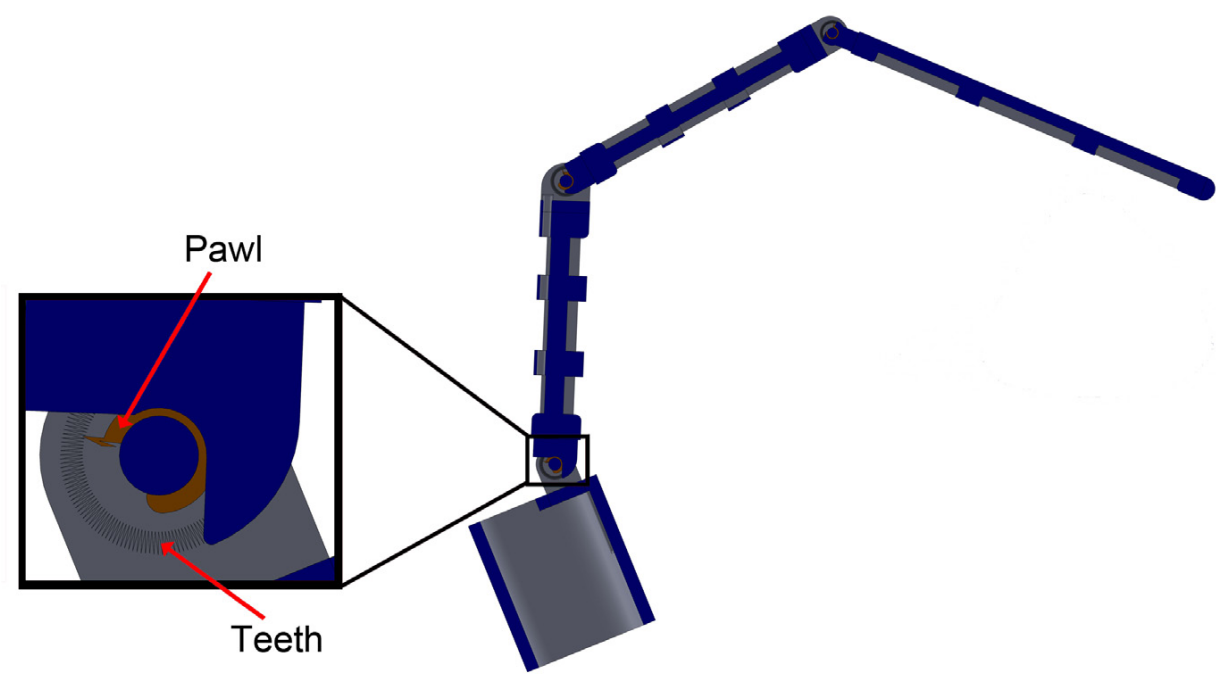

Figure 2.19: Ratchet mechanism for one-directional joints

One important parameter in designing the one-directional joint is the number of teeth. Following calculations are performed to find the proper number of teeth for each joint of the device. These geometrical calculations are considered based on Figure 2.20. As shown in this figure, $\Delta_{1}$ and $\Delta_{2}$ are the generated displacements due to the rotation of the link in the middle and the tip of the link, respectively. The displacement of $\Delta_{1}$ is generated in the position of the $\mathrm{P}$ points. Equation 2.7 and 2.12 can be used to find the values of $\Delta_{1}$ and $\Delta_{2}$, respectively. In these equations, $\theta$ is the angle between two adjacent teeth of the ratchet joint; $\mathrm{R}$ and $\mathrm{L}$ are the radius and length of the link, respectively. 


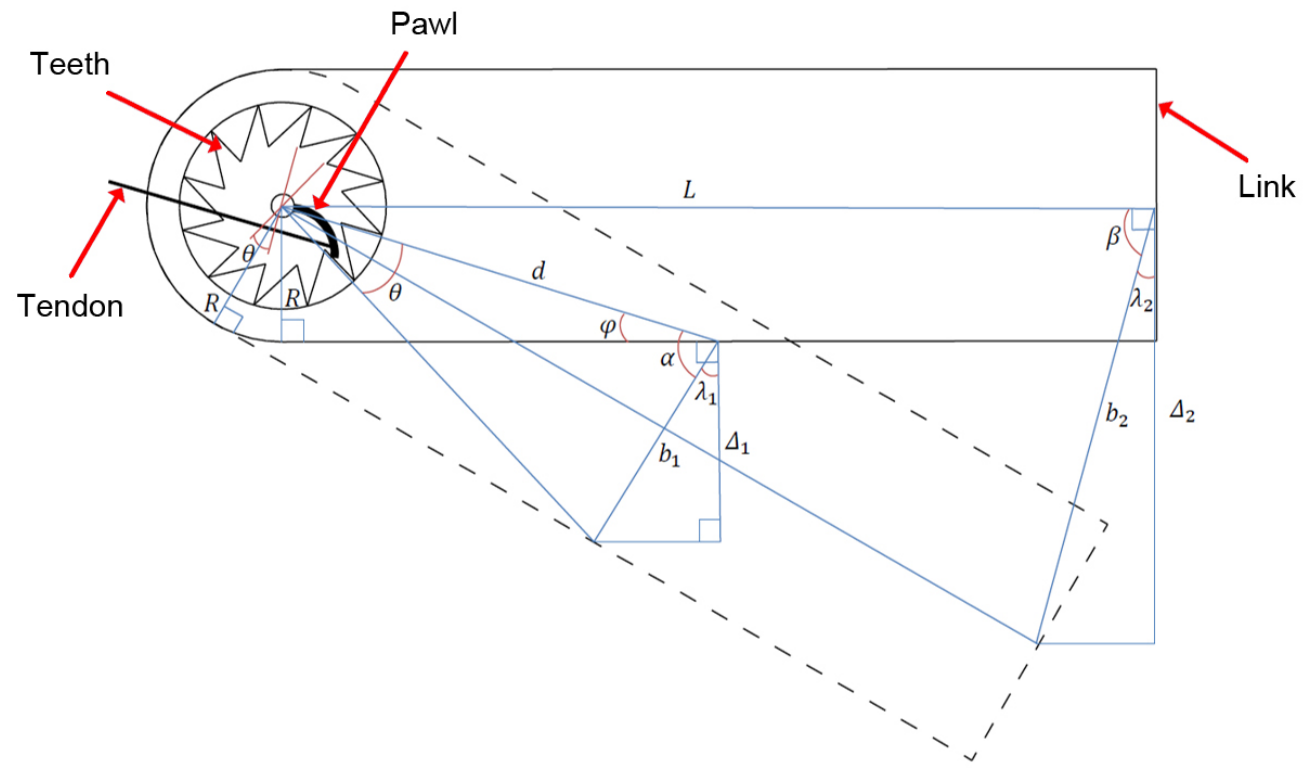

Figure 2.20: Displacement of the link with ratchet one-directional joint

$$
\begin{aligned}
& R^{2}+\left(\frac{L}{2}\right)^{2}=d^{2} \Rightarrow d=\sqrt{R^{2}+\frac{L^{2}}{4}} \\
& b_{1}=2 d \sin \left(\frac{\theta}{2}\right) \\
& \alpha=90-\frac{\theta}{2} \\
& \varphi=\tan ^{-1}\left(\frac{2 R}{L}\right)
\end{aligned}
$$




$$
\lambda_{1}=90-(\alpha-\varphi)=90+\varphi-\alpha
$$

$$
\begin{aligned}
& \Delta_{1}=b_{1} \cos \left(\lambda_{1}\right)=2 d \sin \left(\frac{\theta}{2}\right) \cos \left(\lambda_{1}\right) \\
& =2\left(\sqrt{R^{2}+\frac{L^{2}}{4}}\right) \sin \left(\frac{\theta}{2}\right) \cos (90+\varphi-\alpha) \\
& =2\left(\sqrt{R^{2}+\frac{L^{2}}{4}}\right) \sin \left(\frac{\theta}{2}\right) \cos \left(90+\tan ^{-1}\left(\frac{2 R}{L}\right)-90+\frac{\theta}{2}\right) \\
& =2\left(\sqrt{R^{2}+\frac{L^{2}}{4}}\right) \sin \left(\frac{\theta}{2}\right) \cos \left(\tan ^{-1}\left(\frac{2 R}{L}\right)+\frac{\theta}{2}\right)
\end{aligned}
$$

$$
\Delta_{1}=2 \sqrt{\left(R^{2}+\frac{L^{2}}{4}\right)} \sin \left(\frac{\theta}{2}\right) \cos \left(\tan ^{-1}\left(\frac{2 R}{L}\right)+\frac{\theta}{2}\right)
$$

$$
b_{2}=2 L \sin \left(\frac{\theta}{2}\right)
$$

$$
\beta=90-\frac{\theta}{2}
$$

$$
\lambda_{2}=90-\beta=90-\left(90-\frac{\theta}{2}\right)=\frac{\theta}{2}
$$

$$
\Delta_{2}=b_{2} \cos \left(\lambda_{2}\right)=b_{2} \cos \left(\frac{\theta}{2}\right)=2 L \sin \left(\frac{\theta}{2}\right) \cos \left(\frac{\theta}{2}\right)
$$




$$
\Delta_{2}=2 L \sin \left(\frac{\theta}{2}\right) \cos \left(\frac{\theta}{2}\right)
$$

Increasing the number of teeth results in higher resolution in the generated displacement. However, there are two main constraints in the maximum number of teeth that could be considered in the design. The first limitation is related to the smallest feature that could be fabricated. This factor depends on the fabrication method and material. Some fabrication companies provide ultra-high resolution for 3D printing of the designs with polymer materials (resolution of around $10 \mu \mathrm{m}$ ) [56]. However, the experience shows that usually a minimum feature size of at least three times more than the claimed number by the companies should be considered in the design procedure. Specially if the design includes channels with a high aspect ratio, there is a problem of cleaning the channels from the waste material. This problem can be solved by making the length of channels in the design shorter as much as possible. This can be seen in the design of the device where the gaps are considered in the links to decrease the aspect ratio of the tendon supports. Moreover, the minimum feature size and gap in the design of the device are above $100 \mu \mathrm{m}$ and above $50 \mu \mathrm{m}$, respectively. Therefore, fabrication of the device using high resolution 3D printing technologies is feasible.

The second limitation that needs to be considered is the strength of the small features in the device under the applied force. FEM simulations should be performed to assure the strength of the device under the applied forces.

Table 2.6 provides the generated displacement for each link considering the angle of 5 degrees between two adjacent teeth of the ratchet joint. 
Table 2.6: Generated Displacement by the Links of the Device

\begin{tabular}{|l|c|c|c|c|}
\hline & $\mathbf{R} \mathbf{( m m})$ & $\mathbf{L ~ ( m m )}$ & $\theta$ (degree) & $\left.\Delta_{1} \mathbf{( m m}\right)$ \\
\hline \hline Link 1 & 2 & 29 & 5 & 1.2 \\
\hline Link 2 & 1.5 & 26.1 & 5 & 1.1 \\
\hline Link 3 & 1 & 30.4 & 5 & 1.3 \\
\hline
\end{tabular}

Each joint gets released if a tendon (release tendon) connected to the pawl is pulled. There are tendon supports in the proximal side of the device for the release tendons as shown in Figure 2.21.

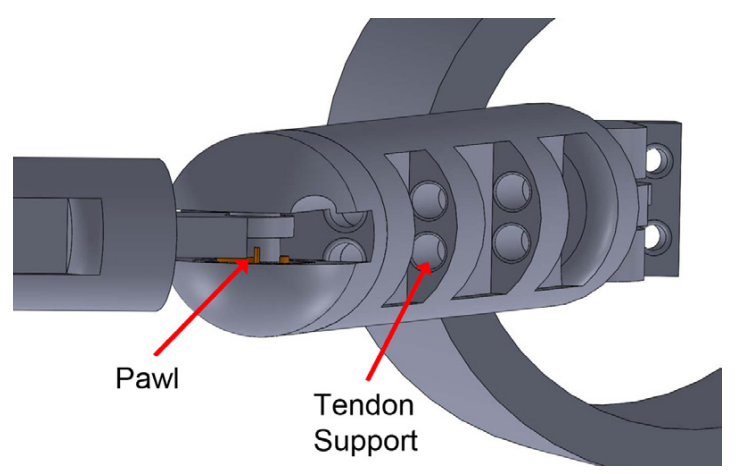

Figure 2.21: The pawl of the joint and tendon supports

In order to increase the safety of the device, a cover will surround the links and joints of the device to prevent any possible blood clots which may be caused by small feature size of the hinges. Moreover, in case of any mechanical failure of the device, the cover will prevent the separated piece from traveling inside the blood circulation system.

\subsection{Grabbing and pulling mechanism}

The six tendons of the device will be placed in six separate channels in the anchor to get engaged and pulled by a steerable catheter. The end parts of the tendons are attached to arrow-shape pieces. The steerable catheter will be introduced to 
the CS ostium and will be engaged with the device. When the catheter is engaged with the device, the six sub-catheters of the steerable catheter are engaged with six tendons of the device. Figure 2.22 depicts one of the sub-catheters and the end part of a tendon. Each sub-catheter is connected to a knob at the handle of the steerable catheter. The surgeon will be able to pull the tendons separately and control the position of each link.

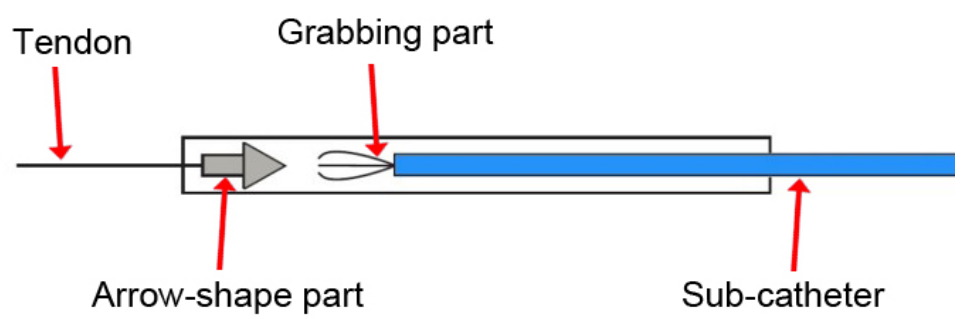

Figure 2.22: The end part of a tendon and a sub-catheter of the steerable catheter

Figure 2.23 shows how the grabbing and pulling mechanism works. The grabbing part of the sub-catheter will get integrated with the arrow-shape part of the tendon. The grabbing part consists of several flexible pieces that will grip the arrow-shape part of the tendon when it is pushed towards the arrow-shape part.

Figure 2.23 shows the steps of grabbing a tendon and pulling it with a sub-catheter. As shown in this figure, the sub-catheter which is introduced into the tendon channel is pushed towards the arrow-shape end of the tendon. The sub-catheter is pushed until the grabbing part grips the arrow-shape end of the tendon. Then, the tendon is pulled by pulling the sub-catheter. A tendon, traveling through the sub-catheter, is attached to the tip of flexible parts of the sub-catheter. With pulling this tendon, the grabbing part gets released and the sub-catheter can be pulled out of the tendon channel. 
(a)

(b)

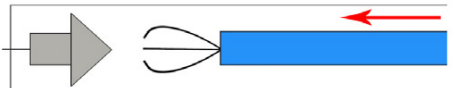

(c)

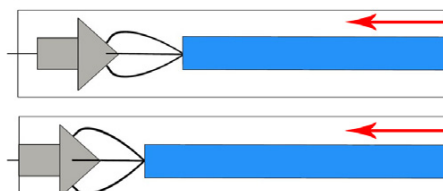

(d)

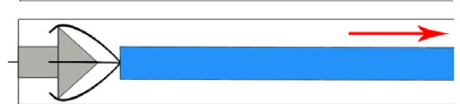

(e)

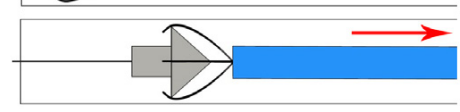

(f)

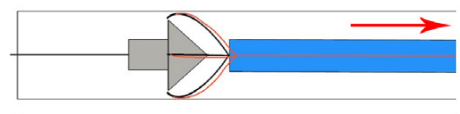

(g)

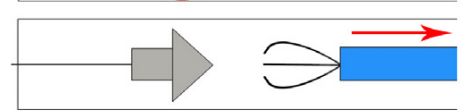

Figure 2.23: Grabbing and pulling a tendon by a sub-catheter of the steerable catheter.

(a) The sub-catheter is pushed towards the arrow-shape end of the tendon. (b) The grabbing part of the sub-catheter reaches to the arrow-shape end of the tendon.

(c) The grabbing part starts to grip the arrow-shape end of the tendon. (d) The grabbing part grips the arrow-shape end of the tendon completely. (e) The tendon is pulled by pulling the sub-catheter. (f) The grabbing part can be released by a tendon which travels through the sub-catheter and is attached to the flexible parts. (g) The sub-catheter is pulled to leave the tendon channel.

\subsection{Engagement mechanism}

The main challenge in introducing the steerable catheter and engaging it with the device is guiding the six sub-catheters to the related tendon channels. Considering that the surgery is an IC operation and the only visual feedback from the procedure is the images taken by X-ray C-arm machine, guiding the sub-catheters to the tendon channels would be a cumbersome procedure. The solution, which is introduced here, is an engagement mechanism to address this issue. This engagement mechanism consists of a male part and a female part. Figure 2.24 depicts the perspective and cross-sectional views of the female part. This part has a guiding rail that will guide 
a channel located on the male part. The male part is shown in Figure 2.25.
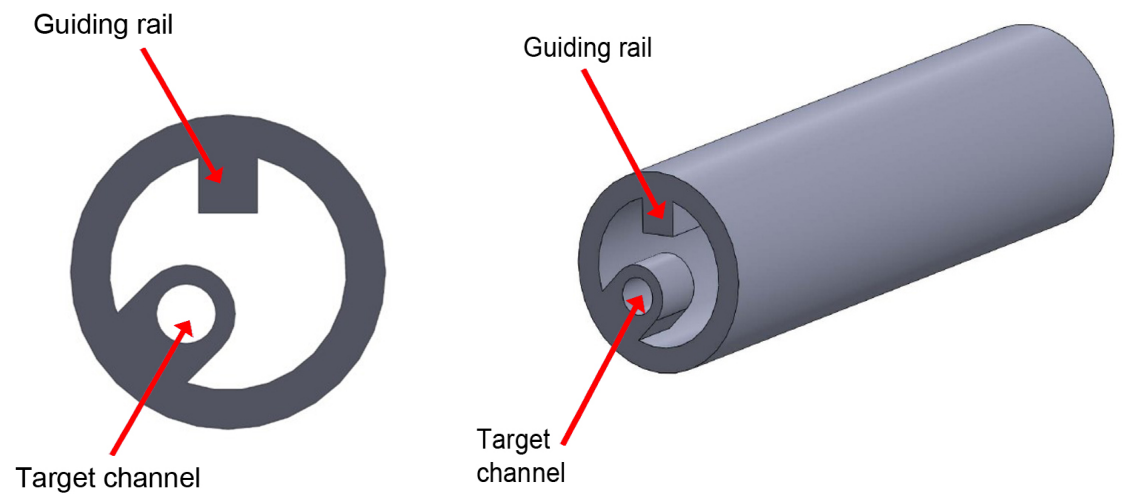

Figure 2.24: Female part of the engagement mechanism. Left: cross-sectional view. Right: Perspective view.
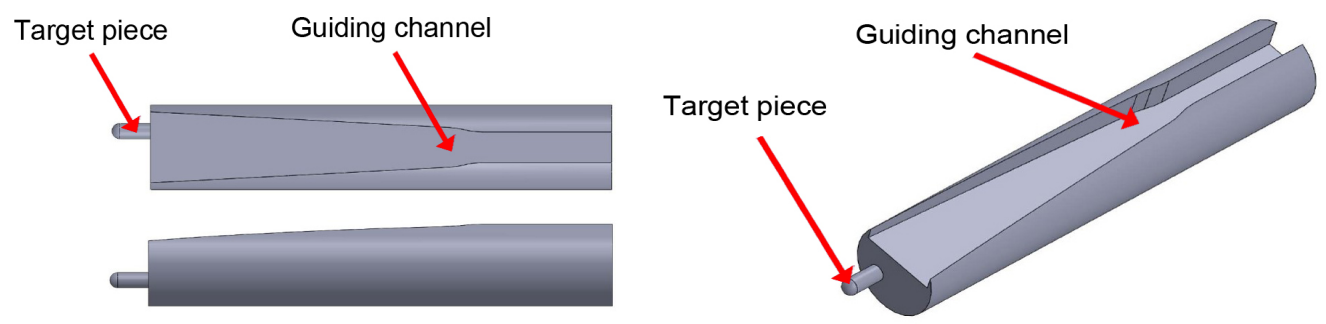

Figure 2.25: Male part of the engagement mechanism. Left: top and side view. Right: Perspective view.

The goal is to guide a small piece (target piece) on the male part to the target channel of the female part. As shown in Figure 2.25, the guiding channel has a wide opening at its distal end and can be easily get engaged with the guiding rail by slight rotation. The guiding channel gets narrower along the male part such that by pushing the male part, the guiding rail will be guided to the narrow part of the channel. Consequently, as the male part is pushed, the target piece will be rotated and will enter the target channel. Figure 2.26 depicts cross sectional view of the final position of the target piece with respect to the target channel. It is noteworthy to mention that as shown in Figure 2.26 there is a gap between the two parts of 
the engagement mechanism such that engagement of them does not block the blood flow. Moreover, a through hole can be considered in the male part of the engagement for preventing any blood flow blockage.

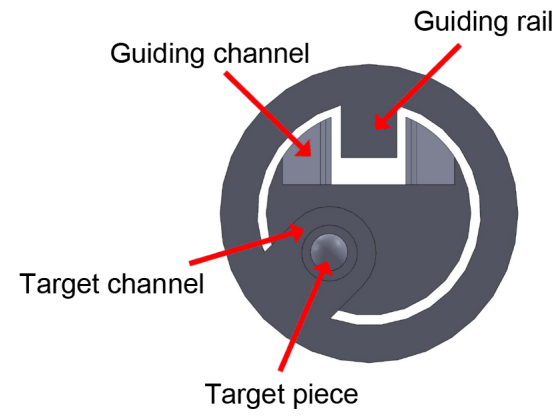

Figure 2.26: The target piece is guided to the target channel by the engagement mechanism.

The introduced engagement mechanism can be used for engaging the steerable catheter and the device such that the six sub-catheters are guided into the related tendon channels. A large-scale prototype of this engagement mechanism is fabricated, and the experimental results are provided in Section 2.8.

The procedure of the device implantation can be explained as follows:

The device is introduced to the CS using a sheath through the femoral vein. The joints of the device are unlocked during the delivery procedure such that the links can move in both directions. This makes delivery of the device easier. When the anchor part of the device is in CS ostium, the sheath is retracted and the anchor which is a self-expandable stent is deployed and the device is fixed inside CS. For controlling the links of the device after implantation, the steerable catheter is introduced. The developed engagement mechanism helps the surgeon engage the steerable catheter with the implanted device easily with translational movements of the catheter. The grabbing and pulling mechanism enables the surgeon to control each link and apply forces to $\mathrm{P}$ points of MA. Tracking of the delivery sheath and steerable catheter 
inside patient body is performed with using X-ray imaging methods.

\subsection{Simulations}

In this section, two validations related to the device are provided. The first validation is conducted using the FEM stress-strain analysis of the device while it is implanted in CS and applying force to MA. This FEM simulation is performed to make sure that the device has enough mechanical strength under the applied force. The second validation is conducted through the CFD simulation of blood inside CS while the device is implanted. This simulation is performed to analyze the safety of the device in terms of not causing blood clots inside CS due to the generated turbulence.

\subsubsection{FEM simulation of the device under applied force}

Considering the amount of force applied by each link to MA, FEM simulation is performed on all three links of the device. The fabrication material is considered as VisiJet EX200 with the properties given in3.7. FEM simulations are performed using COMSOL Multiphysics (COMSOL, Stockholm, Sweden).

The results show that the most vulnerable part of the device under the applied force is the locking mechanism. Figure 2.27 shows the generated stress on the Paw of the one-directional joint while Link 1 applies the force of $0.7 \mathrm{~N}$ (that is twice of the maximum applied force which is around $0.35 \mathrm{~N}$ ). 


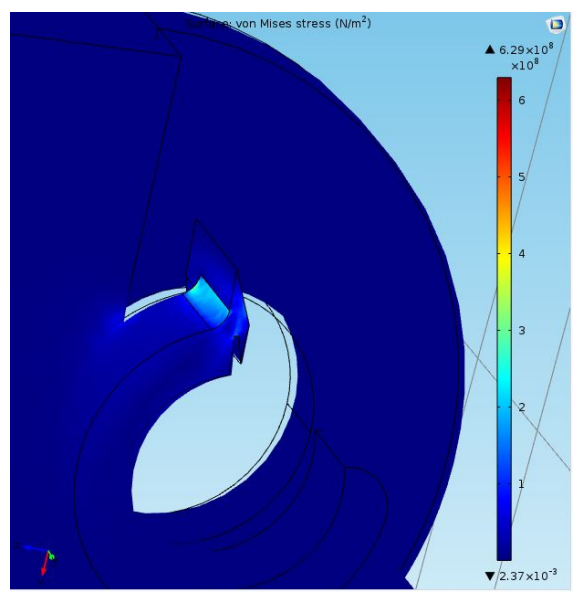

Figure 2.27: Generated stress on the one-directional joint of the Link 1 while the link applies the force of $0.35 \mathrm{~N}$ to the MA

This result shows that the maximum generated stress which is around $629 \mathrm{MPa}$ is much higher than the ultimate tensile strength of the material (42.4 MPa). Based on this analysis, this part of the device cannot be fabricated from the polymer material with such properties. Another option for the material of the device is Titanium alloy (Ti-6Al-4V) which has the ultimate tensile strength of $965 \mathrm{MPa}$. Titanium alloy is a bio-compatible material and can be used in the fabrication of implantable devices [57]. The generated stress in Link 2 and Link 3 are $322 \mathrm{MPa}$ and $218 \mathrm{MPa}$, respectively. Therefore, the device can be fabricated from Titanium alloy.

\subsubsection{CFD simulation of blood inside CS}

Implanting the designed device inside CS will cause blood flow turbulence. Consequently, thrombosis (formation of blood clots) may occur inside CS causing fatal problems [58]. To make sure that the device is safe to be implanted inside CS from the aspect of blood flow, CFD simulation was performed using COMSOL Multiphysics. The 3D model of the CS was developed in SolidWorks software using the geometrical parameters extracted from CT scan analysis. The developed 3D model 
of CS was imported to COMSOL software for CFD simulation. Blood in CS and at normal physiological conditions is a Newtonian liquid and has the constant dynamic viscosity of $3.5 \mathrm{cP}$ (0.0035 Pa.s) [59]. The considered blood pressure inside CS is around $10 \mathrm{mmHg}(1300 \mathrm{~Pa})$ [60]. The mean value of the blood flow in CS is 123 $\mathrm{ml} / \mathrm{min}[61]$. Using this value and the diameter of CS, the velocity of blood flow in CS ostium is calculated as $0.0225 \mathrm{~m} / \mathrm{s}$ which is set as the boundary condition in the simulation. Figure 2.28 shows the simulation results of blood flow streamlines in CS for two different conditions: with the implanted device and without the implanted device. Figure 2.29 depicts the velocity field of blood flow at $\mathrm{P}_{2}$ point for the two different conditions. Simulation results show that with implantation of the device inside CS, the blood flow rate decreases to $116.8 \mathrm{ml} / \mathrm{min}$ which is still in the normal range of blood flow rate inside CS [62]. Therefore, installation of the device inside CS does not cause CS stenosis.

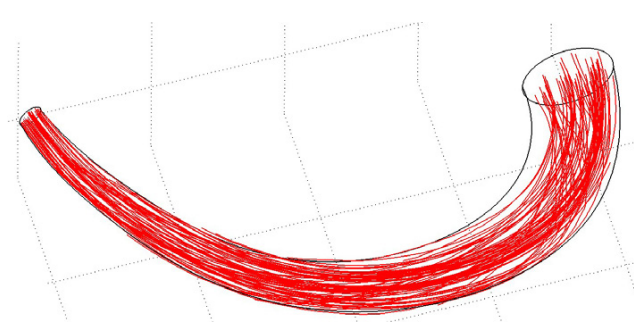

(a)

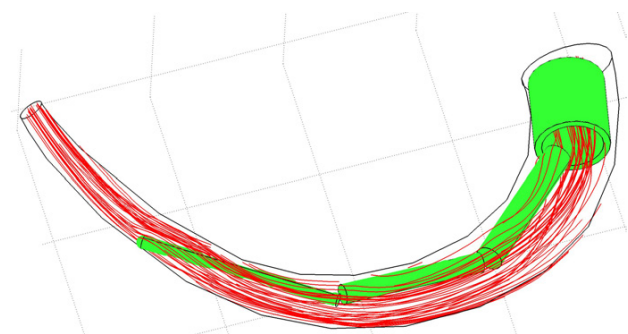

(b)

Figure 2.28: CFD simulation of blood inside CS. (a) Blood flow streamlines in CS without the implanted device. (b) Blood flow streamlines in CS with the implanted device. 


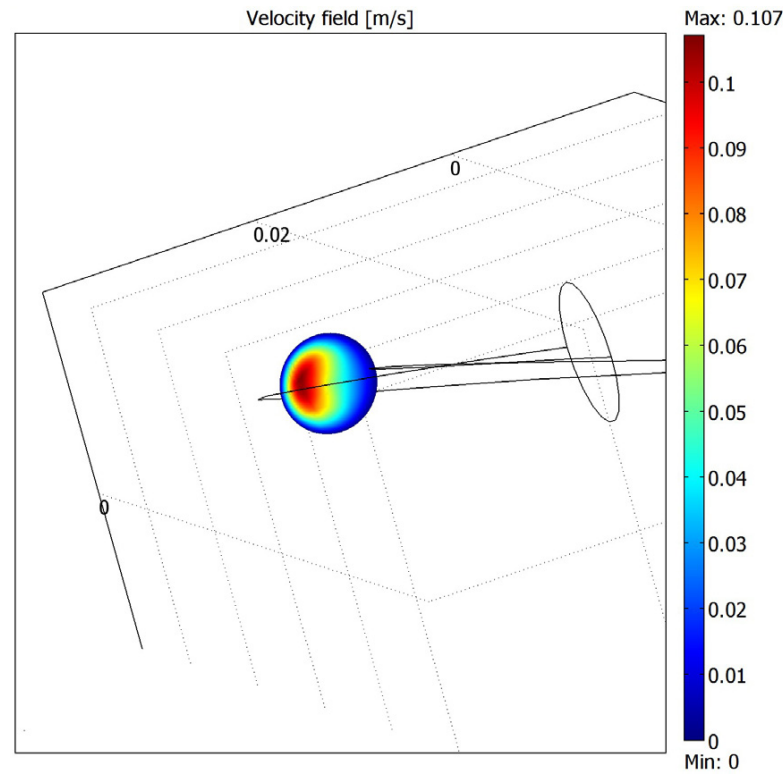

(a)

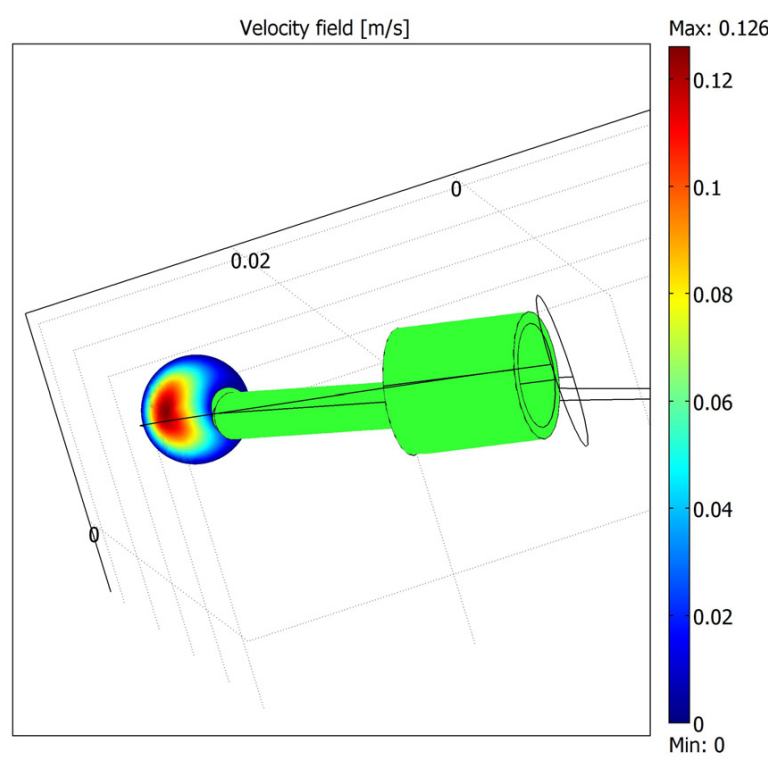

(b)

Figure 2.29: CFD simulation of blood inside CS. (a) Velocity field of blood flow at $\mathrm{P}_{2}$ in $\mathrm{CS}$ without the implanted device. (b) Velocity field of blood flow at $\mathrm{P}_{2}$ in CS with the implanted device.

Simulation results show that the maximum Reynolds number when the device is located inside CS is 264.4. The threshold of the Reynolds number for the blood flow to be a turbulent flow is 2000 [63]. Therefore, implanting the device inside CS is 
safe in terms of not causing thrombosis.

\subsection{Large-scale prototyping of the device}

The large-scale prototype of the catheter-based tunable device was fabricated using 3D printing method (MakerGear LLC, Beachwood, Ohio) with fabrication resolution of $200 \mu \mathrm{m}$ from Polylactic Acid (PLA) material. Figure 2.30 shows the anchor, link, pawl and teeth of the ratchet joints of the fabricated large-scale prototype of the device. Figure 2.31 depicts the assembled large-scale prototype of the catheter-based tunable device. Performed initial experiments on this prototype of the device show that the links can be controlled by pulling the actuation tendons and the onedirectional joints can be released by pulling the release tendons. The function of the large-scale prototype of the device is promising for actual-scale fabrication of the device. Although the actual-scale fabrication of the device has the fabrication challenges because of the small feature size, the considered minimum feature size $(>100 \mu \mathrm{m})$ and minimum gap size $(>50 \mu \mathrm{m})$ in the design of the device are at least five times larger than the fabrication resolution offered by fabrication companies. Therefore, fabrication of the actual-scale prototype of the device is feasible. 
2.7 Large-scale prototyping of the device

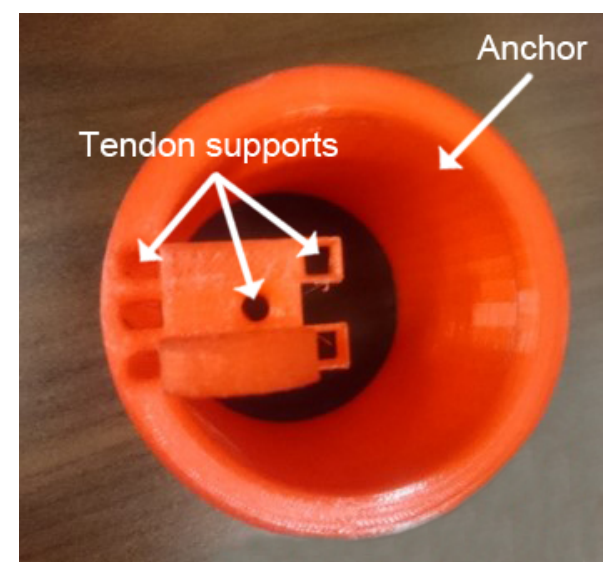

(a)

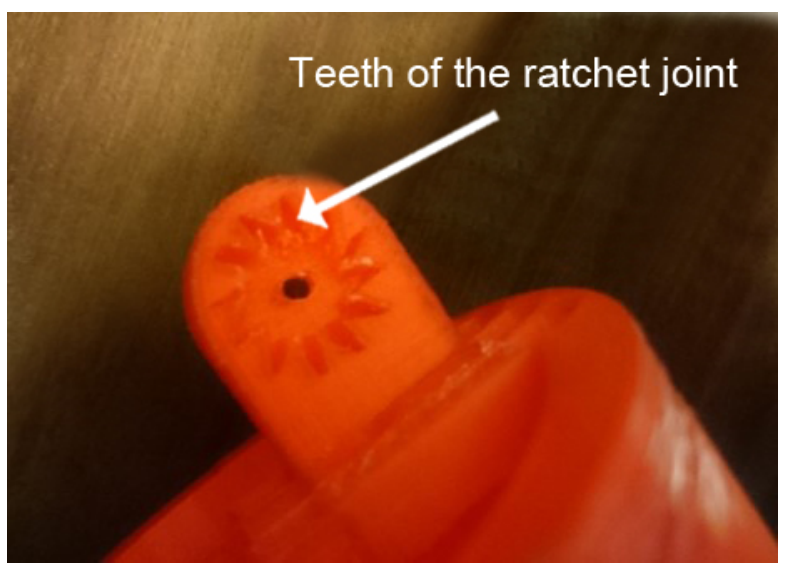

(b)

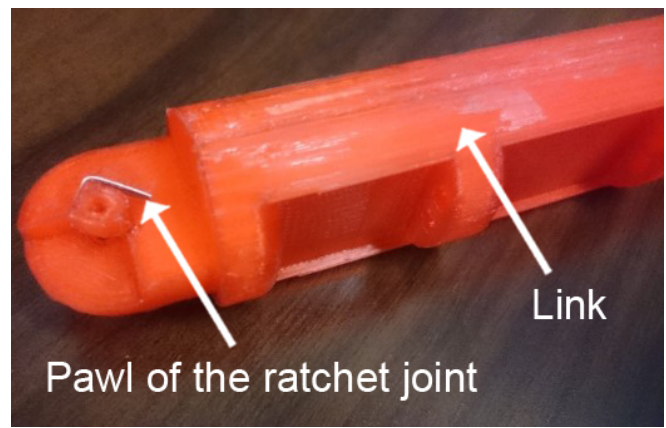

(c)

Figure 2.30: (a) Tendons supports and anchor, (b) Teeth of the ratchet joint, (c) Link and Pawl of the ratchet joint of the large-scale prototype of the device. 


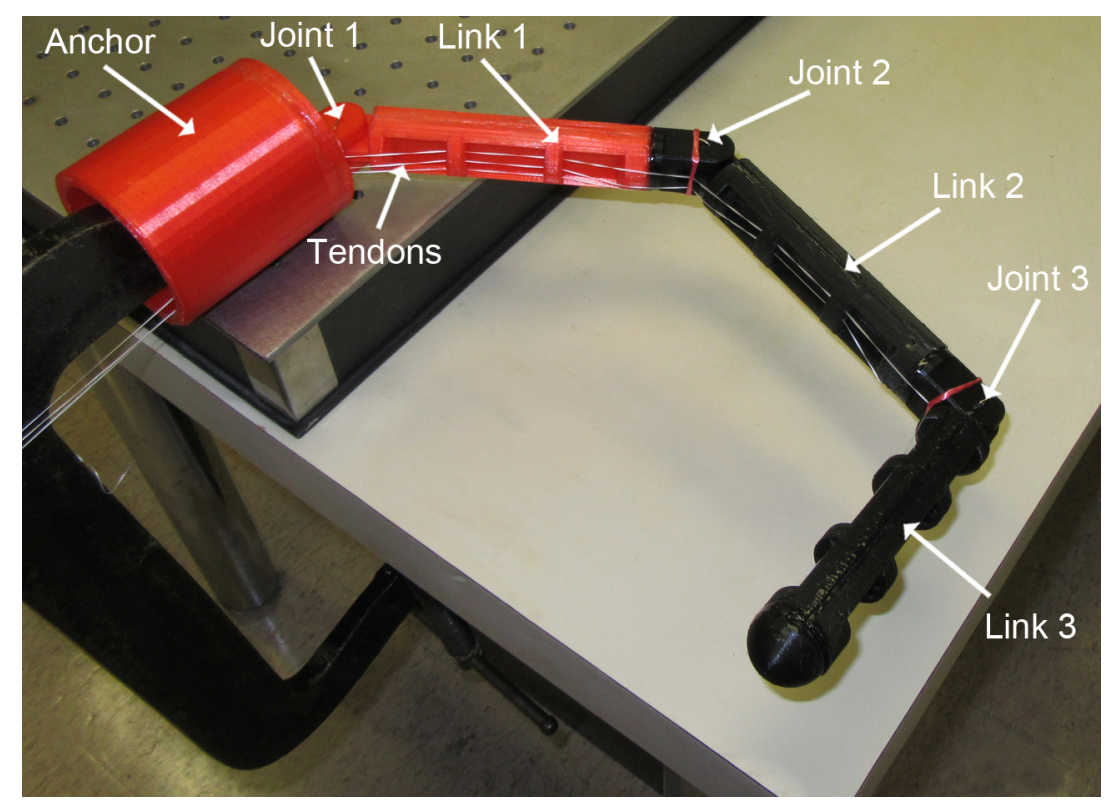

Figure 2.31: Large-scale prototype of the catheter-based tunable device for percutaneous treatment of MR

\subsection{Experimental validation of engagement mechanism}

The engagement mechanism was proposed for the engagement the six sub-catheters of the steerable catheter with the six tendons of the device. A set of experiments is performed to evaluate the success of the design of this engagement mechanism. Ten untrained people were asked to try engaging the two parts of the engagement system.

The female part of the engagement system was fixed on an experimental table, and the male part was attached to the tip of a standard cardiac catheter (Figure 2.32). The diameter of the female part of the mechanism is $11 \mathrm{~mm}$ which is same as the diameter of the anchor in the design. The diameter of the cardiac catheter attached 
to the male part of the mechanism is $6 \mathrm{~mm}(18 \mathrm{Fr})$.

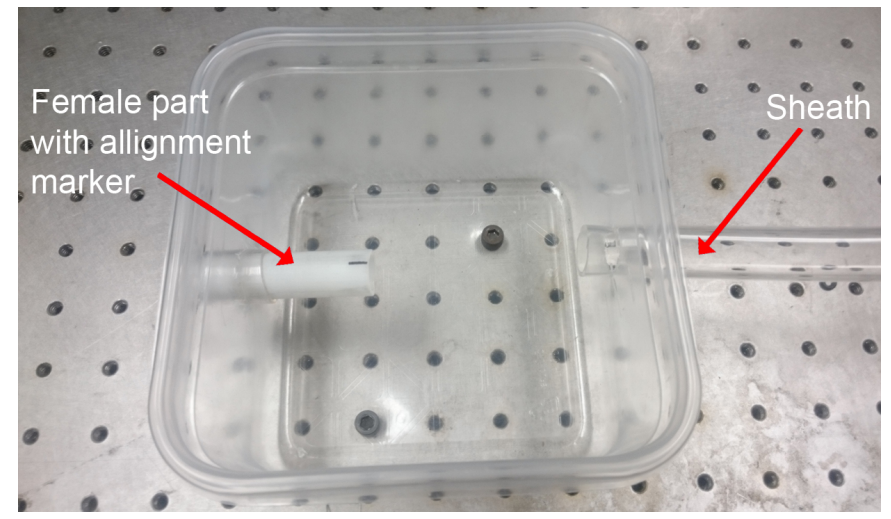

(a)

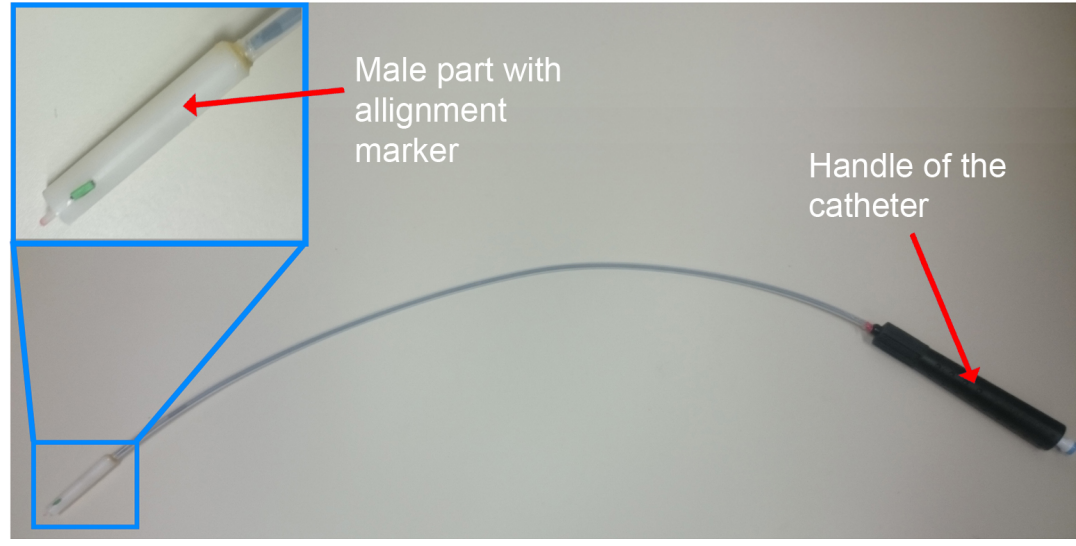

(b)

Figure 2.32: Experimental setup for validation of the designed engagement mechanism.

(a) Female part of the engagement mechanism with the alignment marker is fixed on the experimental table. (b) Male part of the engagement mechanism with alignment marker is attached to the tip of a standard cardiac catheter.

The catheter is introduced through the sheath, and the male part is guided to the female part with translational and rotational movements of the catheter. The alignment markers on the male and female parts help to guide the male part to the female part of the engagement system.

In the IC operation X-ray images are used to help the surgeon perform the surgery. For making the conditions of this experiment similar to the conditions of the op- 
eration, a camera was installed on the top of the experimental setup as shown in Figure 2.33.

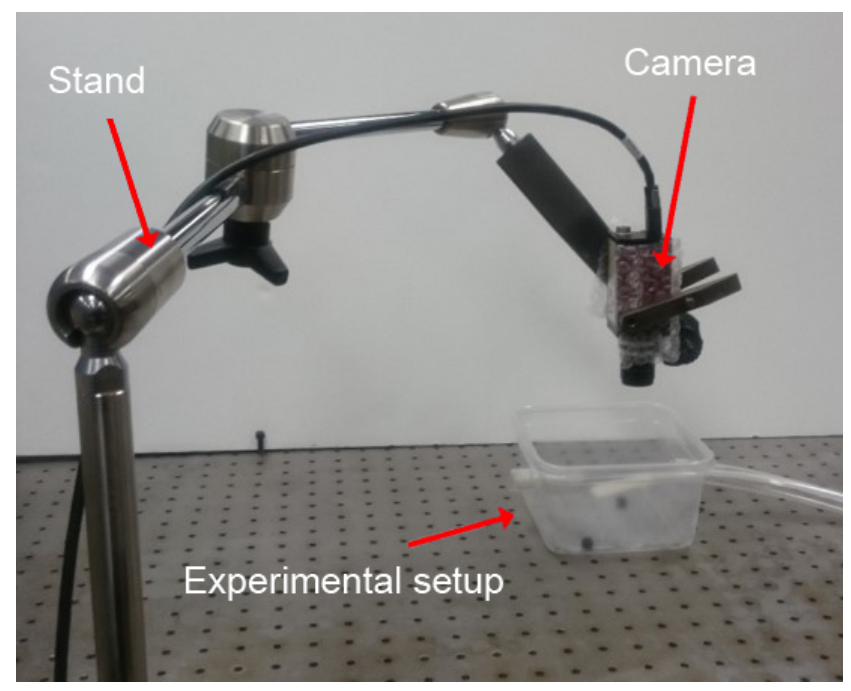

Figure 2.33: Camera is installed on the top of the experimental setup.

For making the picture of the camera similar to X-rays, different filters were tried.

Figure 2.34 shows the original picture with several filters applied on the picture.

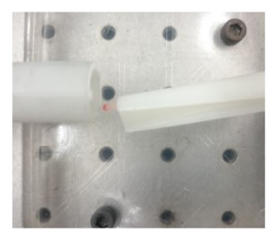

Original image

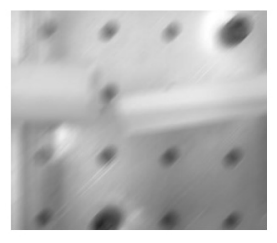

Motion blur

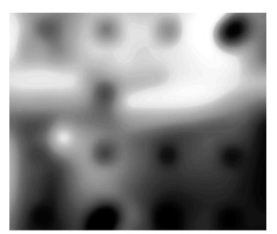

Histogram equalization

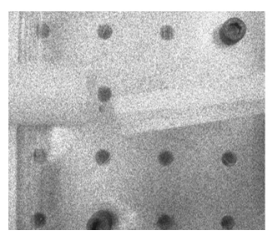

Speckle noise

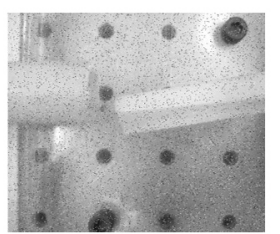

Salt and Pepper

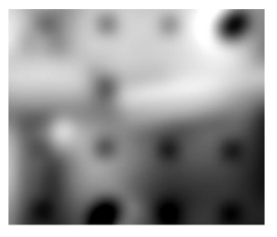

Imadjust 1

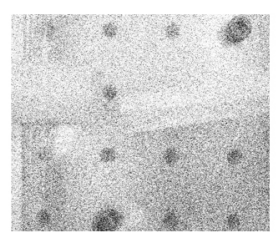

Gausian noise

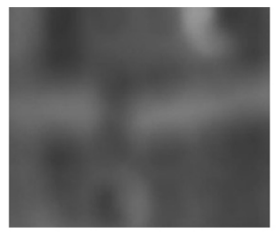

Imadjust 2

Figure 2.34: Different filters are applied on the picture captured by the camera to make it similar to X-ray image.

A monitor was placed in front of the setup so that people use it to manipulate the 
catheter and try to engage the two parts (Figure 2.35).

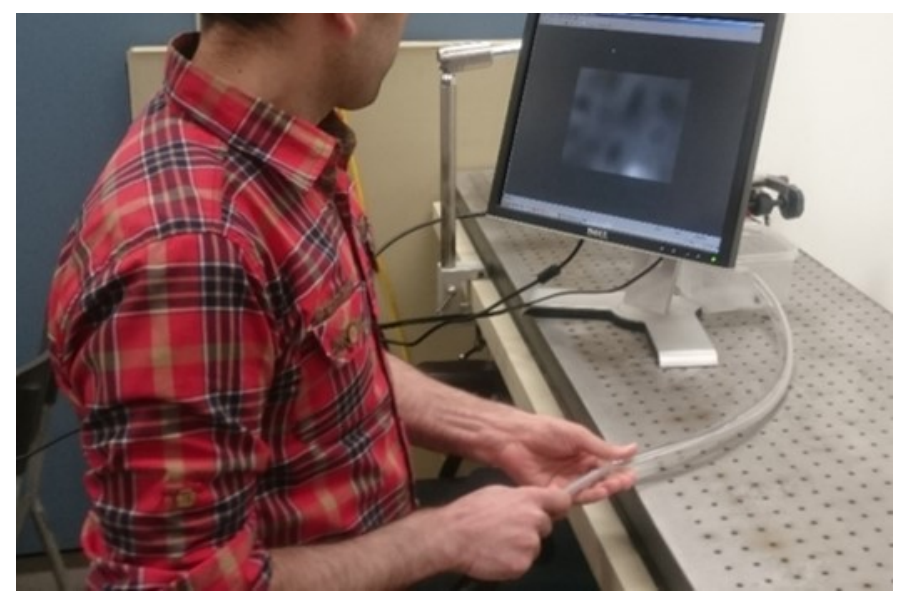

Figure 2.35: People tried to guide the catheter and engage two parts of the mechanism.

Table 2.7 shows the results for ten people performing the experiment. They were asked to perform ten successful experiments. The criteria for success was engaging two parts in less than 2 minutes (the normal time that surgeons need for engagement the steerable catheter with the implanted device). Based on the performed ANOVA gage R\&R test, the appraiser variation is $68.7 \%$ of the system total variation, indicating a large operator-to-operator difference. This maybe is because of the incomplete and imperfect training of the operators. 
Table 2.7: Operation Time (seconds) for Ten People Trying to Engage Two Parts

\begin{tabular}{|c|c|c|c|c|c|c|c|c|c|c|}
\hline \multirow{2}{*}{ Person } & \multicolumn{10}{|c|}{ Trial } \\
\cline { 2 - 12 } & 1 & 2 & 3 & 4 & 5 & 6 & 7 & 8 & 9 & 10 \\
\hline \hline A & 53 & 42 & 19 & 24 & 16 & 12 & 46 & 21 & 18 & 23 \\
\hline B & 84 & 59 & 76 & 112 & 24 & 46 & 59 & 51 & 68 & 34 \\
\hline C & 109 & 78 & 82 & 46 & 32 & 51 & 49 & 53 & 38 & 41 \\
\hline D & 34 & 19 & 46 & 4 & 11 & 5 & 19 & 9 & 26 & 14 \\
\hline E & 79 & 106 & 28 & 49 & 76 & 34 & 81 & 35 & 46 & 31 \\
\hline F & 96 & 117 & 85 & 46 & 11 & 26 & 7 & 34 & 11 & 13 \\
\hline G & 43 & 57 & 21 & 28 & 16 & 42 & 53 & 13 & 11 & 18 \\
\hline H & 85 & 42 & 93 & 75 & 40 & 34 & 26 & 49 & 34 & 64 \\
\hline I & 64 & 16 & 94 & 48 & 37 & 57 & 26 & 102 & 19 & 34 \\
\hline J & 49 & 81 & 38 & 21 & 92 & 27 & 43 & 88 & 62 & 38 \\
\hline
\end{tabular}

Figure 2.36 shows the diagram of the operation time versus the percent of the operations (out of all successful operations performed by ten people). Figure 2.37 depicts the mean procedure time for performing each trial of the experiment. The trend in this figure is decreasing of the procedure time by increasing the trial number which is due to the learning process. Out of all operations carried out by ten people, there were 6 unsuccessful procedures $(t>120 \mathrm{~s})$. Hence, the success rate is calculated as $94.3 \%$.

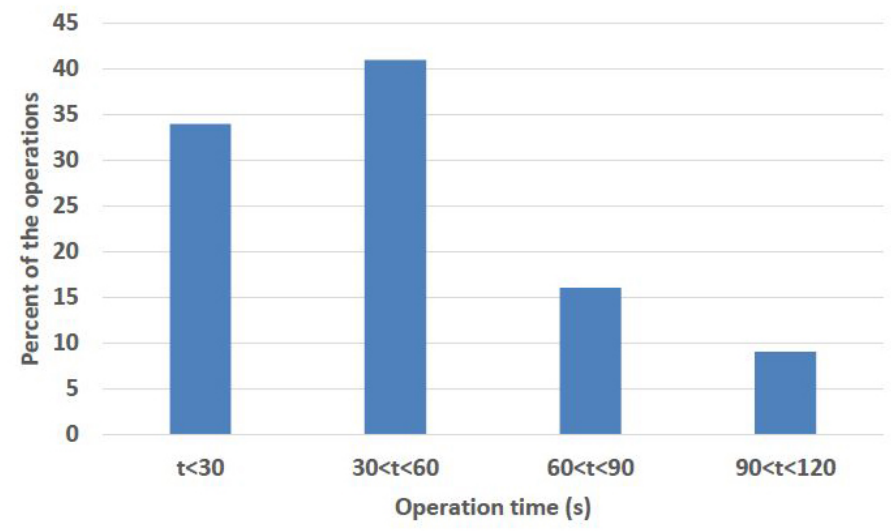

Figure 2.36: Procedure time versus trial number for ten people who performed the operation. 


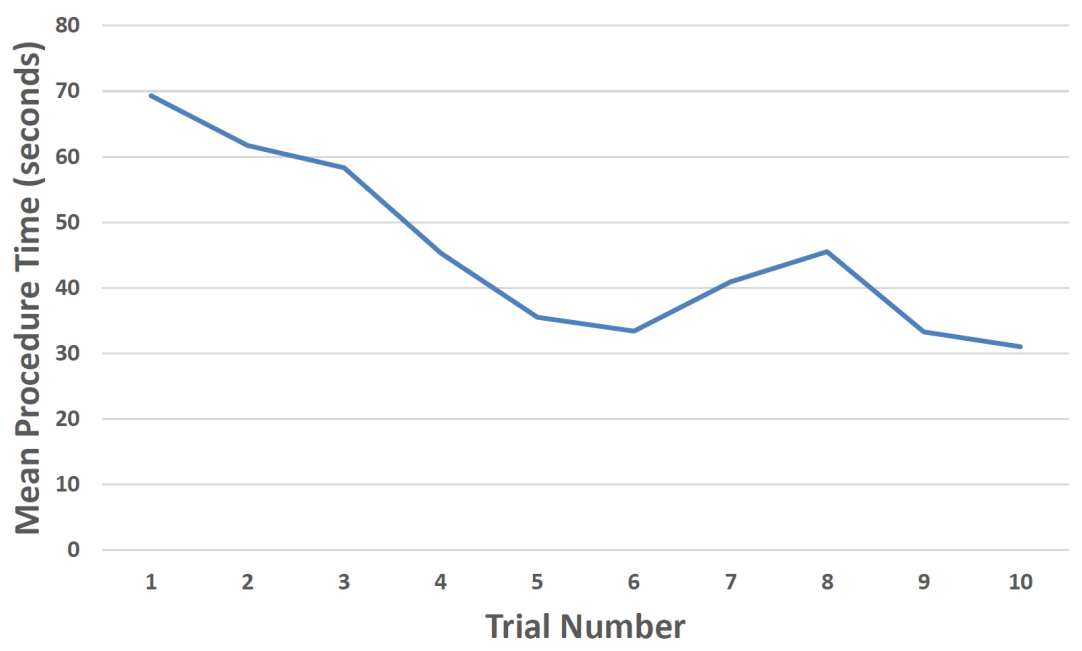

Figure 2.37: Mean procedure time for each trial of the experiment of the engagement mechanism.

\subsection{Summary}

In this chapter of the thesis, a novel tunable catheter-based device for treatment of MR through CS was presented ${ }^{1}$. The device can push each $\mathrm{P}$ point of the posterior leaflet independently while the force applied on LCx artery is minimized. The design of the device was based on the size of the CS obtained from the comprehensive CT scan images analysis. Safety of the device was investigated through FEM stress analysis of the device and CFD analysis of blood inside CS. An engagement mechanism for integrating the device with the steerable catheter was introduced and evaluated with performing the experiments on its large-scale prototype.

\footnotetext{
${ }^{1}$ The patent application of this device is in progress.
} 


\section{Temperature insensitive force/torque sensor for cardiac catheters}

\subsection{Introduction}

In this chapter, the design and prototyping of the catheter force sensor are presented. Moreover, the results of the experiments on the fabricated sensor are provided.

\subsection{Design of the sensing structure}

Two main parts of a force sensor are sensing structure and sensing element. In this project, the focus is on designing a sensing structure that can provide the required specifications for the force measurement in cardiac catheters. For designing such a sensing structure, the requirements of the sensor for the application of ablation cardiac surgery are considered.

The proper range of applied force from an ablation catheter to heart tissue for obtaining optimum outcome of cardiac ablation surgery is reported as 0.1 to $0.4 \mathrm{~N}$ [64]. Although the beating frequency of heart is 1 to $2 \mathrm{~Hz}$, the maximum frequency content of the force signal is higher than this range. The required bandwidth of a sensor for measurement of the contact force is around $4 \mathrm{~Hz}$ [65].

Although the maximum value of the contact force during the ablation surgery is around $0.4 \mathrm{~N}$, the catheter may experience applied force up to $3 \mathrm{~N}$ while passing 
through the hemostatic sheath. Another important feature to be considered for the sensor design is the size of ablation catheters which is between 6 to 15 Fr. The designed sensor should be in this range of size to be integrated with the catheters. This limitation is a structural constraint for the sensor.

Table 3.1 lists the requirements that need to be considered in designing the force sensor for cardiac ablation catheters.

Table 3.1: Requirements of the Force Sensor for Application of Cardiac Ablation Surgery

\begin{tabular}{|l|l|}
\hline Parameter & Requirement for cardiac ablation surgery \\
\hline \hline Size of the catheter & $6-15 \mathrm{Fr}$ \\
\hline Range of applied contact force & $0.1-0.4 \mathrm{~N}$ \\
\hline Maximum contact force & $3 \mathrm{~N}$ \\
\hline Bandwidth & $\sim 4 \mathrm{~Hz}$ \\
\hline
\end{tabular}

The characteristics of the force sensor designed for cardiac catheters include safety, MRI compatibility, and robustness against body fluid. Functional requirement of the sensor is the measurement of force and torque applied to the catheter's distal end without temperature cross-sensitivity.

A schematic view of the final design of the sensing structure for the force sensor is presented in Figure 3.1 (a). 


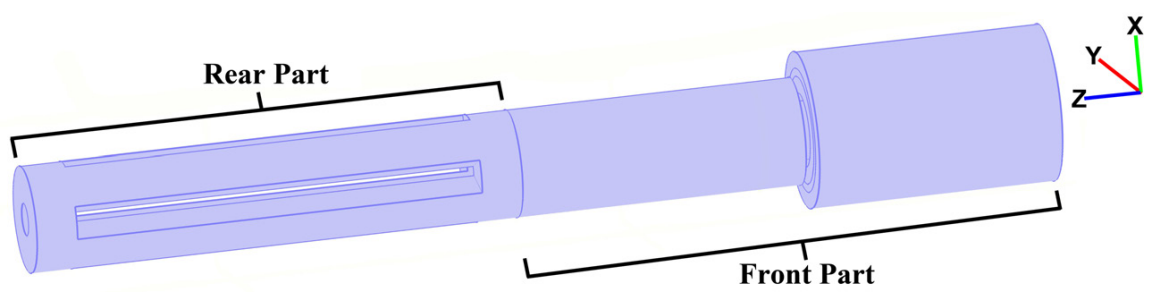

(a)

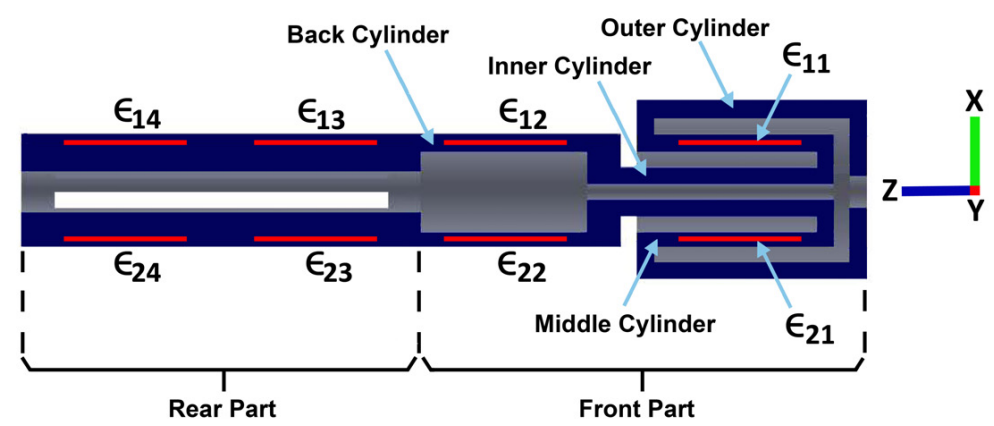

(b)

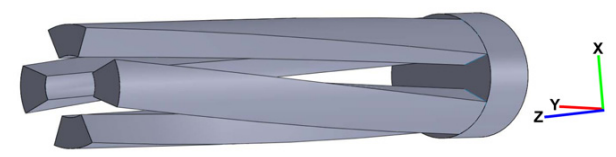

(c)

Figure 3.1: Sensing structure of the sensor, the front and rear parts of the structure.

(a) Sensing structure of the sensor and its front and rear parts. (b) Longitudinal cross sectional view of the sensing structure including strain gauges. (c) Twist of the sensing structure results in twist and bending of the beams in the rear part.

The sensing structure of the sensor includes two main parts, the front part designed for sensing the axial and lateral forces applied to the tip of cardiac catheters and the rear part designed for measuring the generated torque in the distal end of the catheter. Longitudinal cross-sectional view of the front part is shown in Figure 3.1 (b). The front part of the sensing structure consists of four hollow cylinders which are connected to each other. Three of these cylinders (inner, middle and outer cylinders) are located in the distal end of the front part while the fourth cylinder (back cylinder) is located in the proximal end of the front part. Applied forces and 
torques to the catheter can be sensed by measuring the generated strains in different areas of the sensing structure. Applying forces in the axial and lateral directions to the sensing structure generates positive and negative strains in different regions of the front part. The rear part of the structure is a hollow cylinder with four longitudinal gaps. These gaps are placed in the design such that applied torque in the catheter generates positive and negative longitudinal strains in different areas of the beams located in the rear part. The longitudinal strains are generated because the beams experience bending when the whole structure is twisted. This fact is shown in Figure 3.1 (c). As shown in this figure, the tips of the beams move in XY plane indicating that the beams are bent due to the twist of the sensing structure. There is a through hole in the center of the sensing structure for letting wires and tendons of the catheter pass through the structure.

\subsection{Simulation analysis of the sensing structure}

The sensing structure of the sensor is simulated with the Finite Element Method (FEM) using COMSOL Multiphysics (COMSOL, Stockholm, Sweden) for analyzing the behavior of the structure and optimizing its geometry. The dimensions of the sensing structure are selected and optimized for the application of cardiac ablation catheters. The total length of the structure is $25.4 \mathrm{~mm}$, and the outer diameters of the structure in the front part and the rear part are $5.5 \mathrm{~mm}$ and $3.5 \mathrm{~mm}$, respectively. The properties of fabrication material (discussed in section 3.7) have been considered in the simulations.

Simulation results for the applied axial and lateral forces are presented in Figure 3.2 (a) and (b). This figure shows that compressive and tensile strains are generated in the front part of the sensing structure due to the applied axial forces. Applying 
lateral forces to this sensing structure also generates positive and negative strains in different regions of the structure. In the FEM simulation, the axial force in the $Z$ direction $\left(F_{z}\right)$ is $0.25 \mathrm{~N}$. The lateral force in this simulation is considered in $Y$ direction $\left(F_{y}\right)$ with a value of $0.25 \mathrm{~N}$. Simulation results for the applied torque, presented in Figure 3.2 (c), show that compressive and tensile strains are generated in the rear part of the sensing structure because of an applied torque of $0.9 \mathrm{Nmm}$ in the $Z$ direction $\left(T_{z}\right)$. Based on the FEM simulation results, the axial and lateral forces applied to the sensing structure for generating the stress of $42.4 \mathrm{MPa}$ are 17.86 $\mathrm{N}$ and $1.41 \mathrm{~N}$, respectively. These values are the maximum axial and lateral forces that can be applied to the structure without mechanical damage. The maximum torque that can be applied to this sensing structure without the mechanical break is $2.81 \mathrm{Nmm}$. 


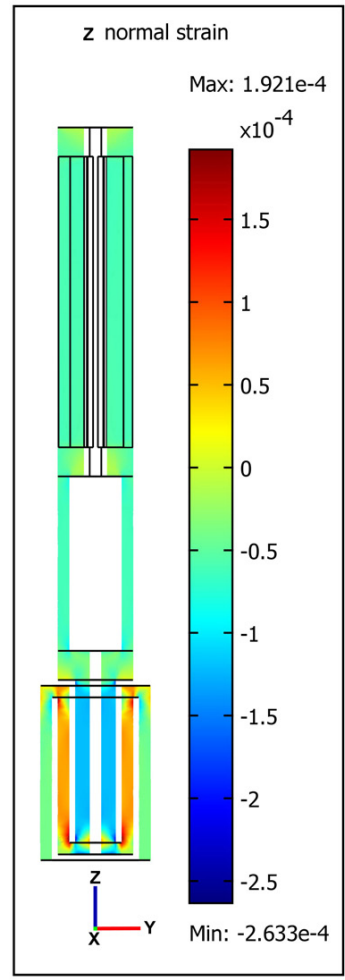

(a)

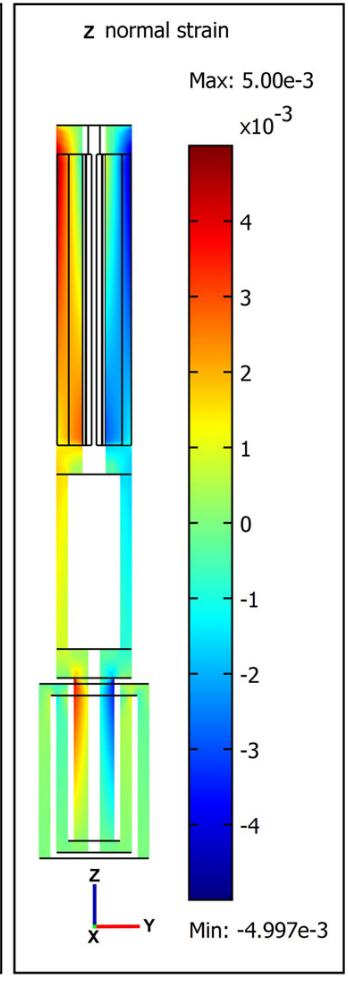

(b)

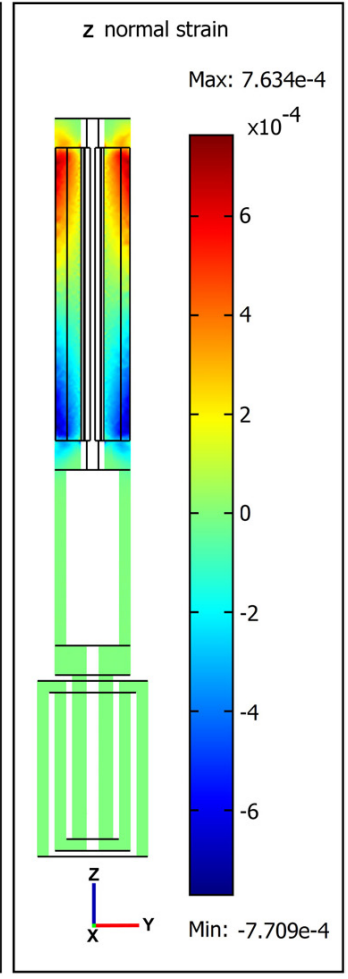

(c)

Figure 3.2: FEM simulation results show the generated strain in the sensing structure due to the applied forces and torque to the structure.

(a) Generated strain because of the applied axial force (Fz). (b) Generated strain because of the applied lateral force (Fy). (c) Generated strain because of the applied torque $(\mathrm{Tz})$.

\subsection{Validation of the sensing structure}

For validating the sensing structure and the fact of generating strains with opposite signs in the structure under applied forces and torques, a large-scale prototype of the sensing structure is fabricated. The prototype is fabricated using 3D printing method from Acrylonitrile Butadiene Styrene (ABS) polymer material. For measuring the generated strains in the structure, eight resistive strain gauges are attached to different parts of the structure. Figure 3.3 shows the fabricated prototype of the sensing structure with the numbered strain gauges. 




(a)

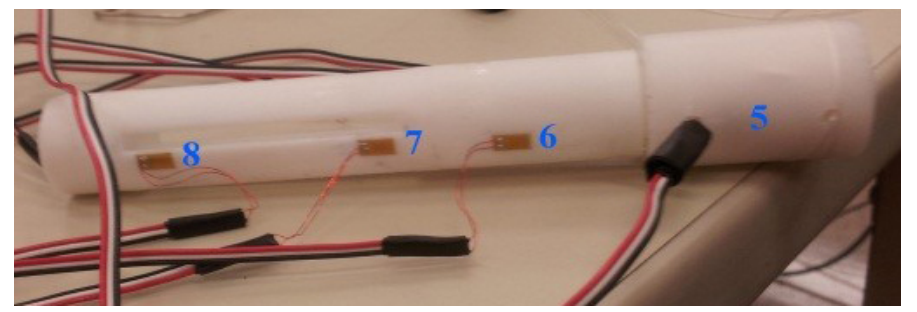

(b)

Figure 3.3: Fabricated large-scaled prototype of the sensing structure with resistive strain gauges, (a) strain gauges number 1 to 4, (b) strain gauges number 5 to 8 .

Axial and lateral force and torque are applied to the structure using standard weights. The amount of generated strain is measured by a digital strain indicator (model P3, Micro Measurements) with accuracy of $0.1 \%$ and measurement resolution of $1 \mu \epsilon$. The experimental setup for applying lateral force to the sensing structure is shown in Figure 3.4. 
3.4 Validation of the sensing structure

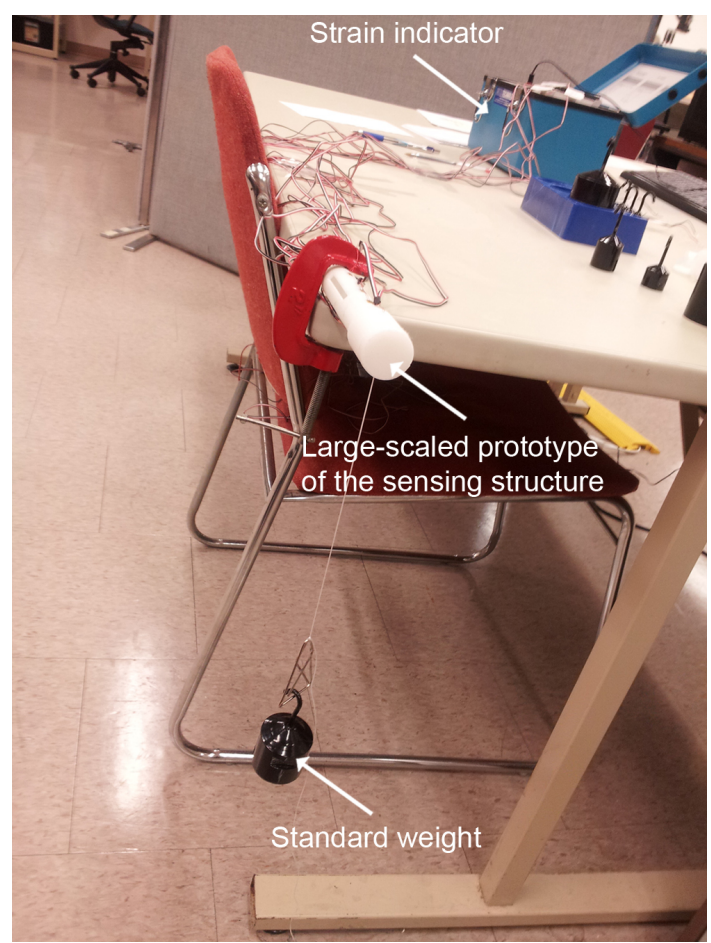

Figure 3.4: Experimental setup for applying lateral force to the large-scaled prototype of the sensing structure

Figure 3.5 provides the measured strains generated in the structure under different axial forces, lateral forces and torques. 


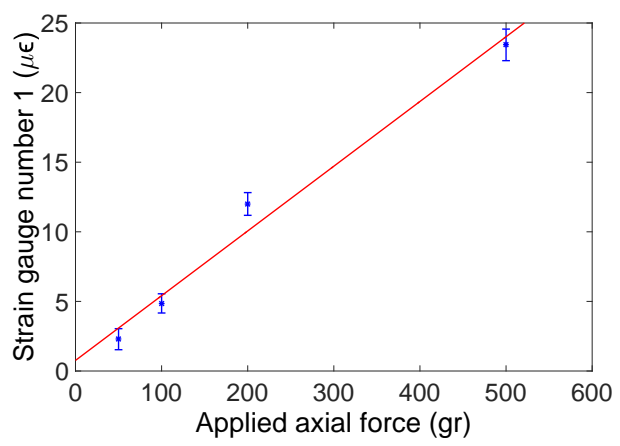

(a)

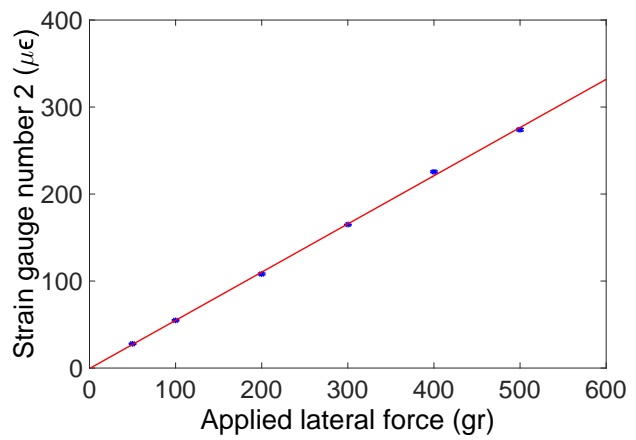

(c)

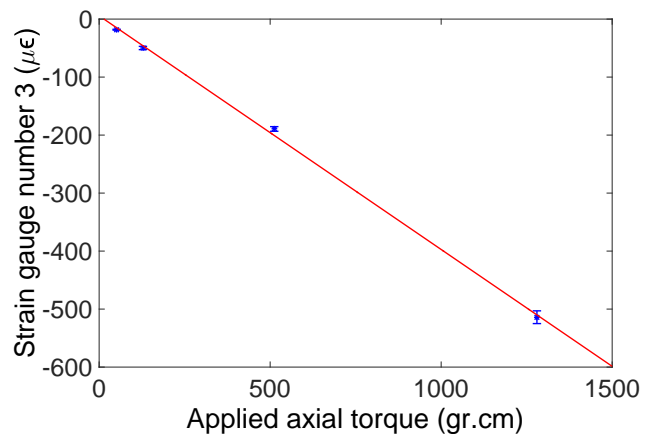

(e)

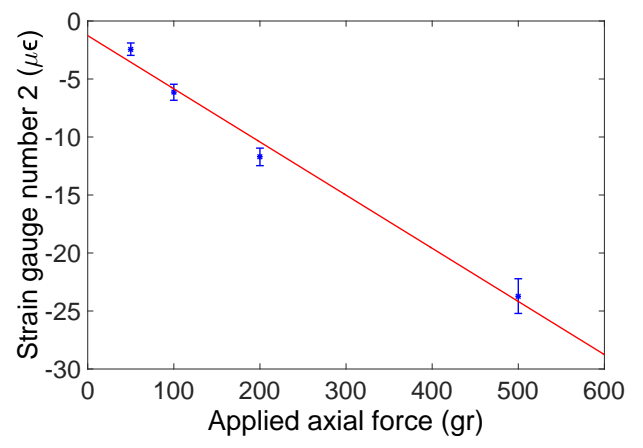

(b)

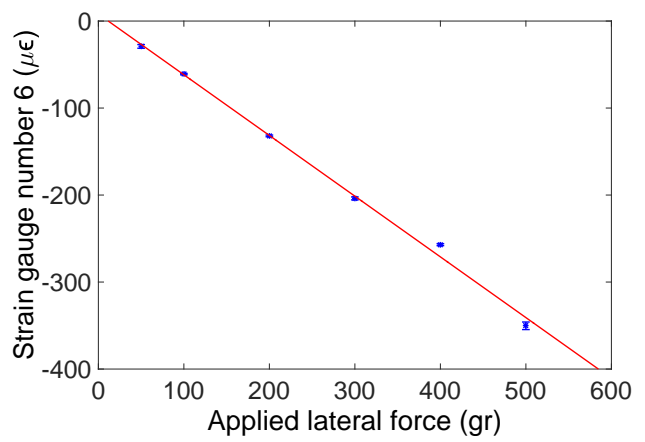

(d)

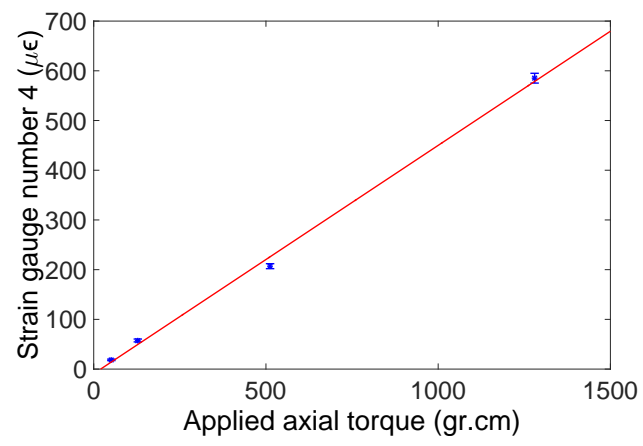

(f)

Figure 3.5: Generated strains in the sensing structure under applied axial force, lateral force and torque.

As shown in Figure 3.5 (a) and (b), resistive strain gauges number 1 and number 2 measure the strains with opposite signs under the applied axial force. Applied lateral force generates the strains with opposite signs as strain gauges number 2 and number 6 measure them (Figure 3.5 (c) and (d)). It is also shown in Figure 3.5 (e) and (f) that strain gauge number 3 experiences compressive strain while strain 
gauge number 4 experiences tensile strain due to the applied torque.

\subsection{Sensing method}

The method of sensing in the proposed force/torque sensor is based on differential measurement. Eight strain gauges can measure the generated strains in the front part of the sensing structure. Configuration of these strain gauges is shown in Figure 3.6 (a) and (b).

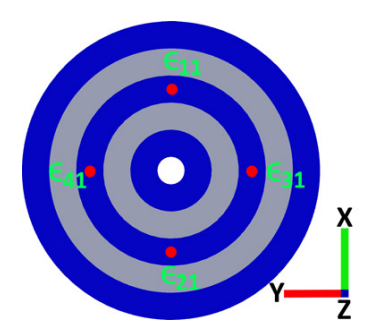

(a)

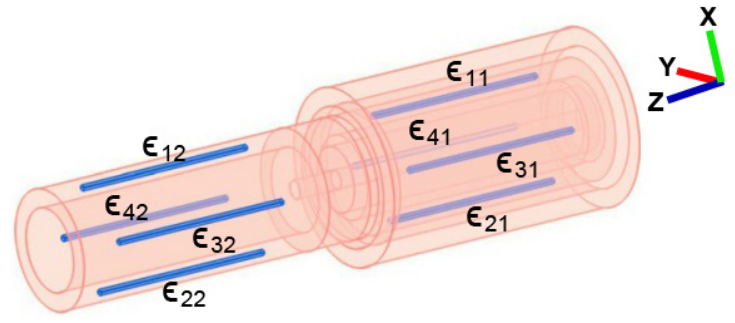

(b)

Figure 3.6: Configuration of the strain gauges in the front part of the sensing structure.

(a) Cross section of the front part at the middle of three distal cylinders showing the position of the strain gauges. (b) 3D transparent view of the front part including the strain gauges.

Strain gauges $\epsilon_{11}, \epsilon_{21}, \epsilon_{31}$ and $\epsilon_{41}$ placed in the middle hollow cylinder of the front part, experience positive strains due to the applied axial force $\left(F_{z}\right)$ while strain gauges $\epsilon_{12}, \epsilon_{22}, \epsilon_{32}$ and $\epsilon_{42}$ placed in the back hollow cylinder of the front part, experience negative strains. The amount of applied axial force can be calculated from the strains measured by these eight strain gauges using the following formula:

$$
F_{z}=\frac{E A}{8}\left(\epsilon_{11}+\epsilon_{21}+\epsilon_{31}+\epsilon_{41}-\epsilon_{12}-\epsilon_{22}-\epsilon_{32}-\epsilon_{42}\right),
$$

where $E$ is the Tensile Modulus of the material and $A$ is the cross sectional area of 


\subsection{Sensing method}

the middle hollow cylinder and the back hollow cylinder which contain the strain gauges. It is noteworthy to mention that the inner and outer diameters of the middle hollow cylinder and the back hollow cylinder are considered equal in design of the sensing structure.

Applying lateral forces in $X$ direction generates strains in the location of $\epsilon_{12}$ and $\epsilon_{22}$ with opposite signs. Similarly, the applied lateral forces in $Y$ direction generate strains in the location of $\epsilon_{32}$ and $\epsilon_{42}$ with opposite signs. Measurement of the generated strains in these strain gauges can be used for calculation of the applied lateral forces through the following formulas:

$$
\begin{aligned}
& F_{y}=\frac{E I}{2 d l}\left(\epsilon_{32}-\epsilon_{42}\right), \\
& F_{x}=\frac{E I}{2 d l}\left(\epsilon_{22}-\epsilon_{12}\right) .
\end{aligned}
$$

In 3.2 and $3.3, I$ is the section moment of inertia of the back hollow cylinder, $d$ is the distance from the central axis ( $Z$ axis) to the acting plane of the strain gauges and $l$ is the distance from the strain gauges to the tip of the catheter where the lateral force is applied.

Eight strain gauges can be embedded in the rear part of the sensing structure as shown in Figure 3.7 to measure the generated strains due to the applied torque $\left(T_{z}\right)$. Longitudinal cross sectional view of the sensing structure including the strain gauges in the rear and front parts of the structure is shown in Figure 3.6 (b). Temperature independent measurement of $T_{z}$ can be achieved by measuring any pair of the strain gauges from $\left[\epsilon_{13}, \epsilon_{23}, \epsilon_{33}, \epsilon_{43}\right]$ group and $\left[\epsilon_{14}, \epsilon_{24}, \epsilon_{34}, \epsilon_{44}\right]$ group. Strain gauges 


\subsection{Sensing method}

of these two groups experience strains with opposite signs. 3.4 can be used for calculating the applied torque based on the measured strains from all FBGs of the proximal part. In this equation, $P$ is a constant value extracted from FEM simulation results with the value of $1.25 \times 10^{3} \mathrm{Nmm}$, approximately. The generated bending $\left(T_{x}\right.$ and $\left.T_{y}\right)$ in MIS instruments are mainly due to lateral forces applied to the tip of the instrument and can be measured from $F_{x}$ and $F_{y}$.

$$
T_{z}=P\left(\epsilon_{13}+\epsilon_{23}+\epsilon_{33}+\epsilon_{43}-\epsilon_{14}-\epsilon_{24}-\epsilon_{34}-\epsilon_{44}\right) .
$$

Figure 3.7: Configuration of the strain gauges in the rear part of the sensing structure.

The following calculations show that the sensing method provides a thermal insensitive measurement without any crosstalk between the measurement channels. The assumed values of generated strains $\epsilon_{z i j}, \epsilon_{x i j}, \epsilon_{y i j}$ and $\epsilon_{T i j}(i, j=1,2,3,4)$ are listed in Table 3.2 which are components of strain $\epsilon_{i j}$ under applied $F_{z}, F_{x}, F_{y}$ and $T_{z}$, respectively. It is noteworthy to mention that some elements of this table are zero because of the arrangement of FBGs in the sensing structure. For instance, strain gauge $\epsilon_{21}$ does not experience any generated strain under the applied lateral force $F_{y}$ because of lying in the respective neutral plane. Therefore, $\epsilon_{y 21}$ is zero in the 
provided table.

Table 3.2: Generated Strains in FBGs under Applied Axial and Lateral Forces, Torque and Temperature Variation

\begin{tabular}{|c|c|c|c|c|}
\hline Strain & $\epsilon_{z i j}$ & $\epsilon_{y i j}$ & $\epsilon_{x i j}$ & $\epsilon_{T i j}$ \\
\hline \hline$\epsilon_{11}$ & $B$ & 0 & $-M$ & 0 \\
\hline$\epsilon_{21}$ & $B$ & 0 & $M$ & 0 \\
\hline$\epsilon_{31}$ & $B$ & $D$ & 0 & 0 \\
\hline$\epsilon_{41}$ & $B$ & $-D$ & 0 & 0 \\
\hline$\epsilon_{12}$ & $-B$ & 0 & $-N$ & 0 \\
\hline$\epsilon_{22}$ & $-B$ & 0 & $N$ & 0 \\
\hline$\epsilon_{32}$ & $-B$ & $G$ & 0 & 0 \\
\hline$\epsilon_{42}$ & $-B$ & $-G$ & 0 & 0 \\
\hline$\epsilon_{13}$ & $-C$ & 0 & $-R$ & $W$ \\
\hline$\epsilon_{23}$ & $-C$ & 0 & $R$ & $W$ \\
\hline$\epsilon_{33}$ & $-C$ & $H$ & 0 & $W$ \\
\hline$\epsilon_{43}$ & $-C$ & $-H$ & 0 & $W$ \\
\hline$\epsilon_{14}$ & $-C$ & 0 & $-J$ & $-W$ \\
\hline$\epsilon_{24}$ & $-C$ & 0 & $J$ & $-W$ \\
\hline$\epsilon_{34}$ & $-C$ & $K$ & 0 & $-W$ \\
\hline$\epsilon_{44}$ & $-C$ & $-K$ & 0 & $-W$ \\
\hline
\end{tabular}

If only a pure axial force applied to the sensor exists and there is no twist or other components of the contact force, 3.1 can be rewritten as:

$$
F_{z}=\frac{E A}{8}(4 B-(-4 B))=\frac{E A}{8}(8 B)=E A B
$$

If temperature variation, twist and other components of the contact force are applied to the sensor, 3.1 can be written as: 


$$
\begin{aligned}
& F_{z}=\frac{E A}{8}\left[\left(\epsilon_{z 11}+\epsilon_{x 11}+\epsilon_{\Delta T}\right)+\left(\epsilon_{z 21}+\epsilon_{x 21}+\epsilon_{\Delta T}\right)\right. \\
& +\left(\epsilon_{z 31}+\epsilon_{y 31}+\epsilon_{\Delta T}\right)+\left(\epsilon_{z 41}+\epsilon_{y 41}+\epsilon_{\Delta T}\right) \\
& -\left(\epsilon_{z 12}+\epsilon_{x 12}+\epsilon_{\Delta T}\right)-\left(\epsilon_{z 22}+\epsilon_{x 22}+\epsilon_{\Delta T}\right) \\
& \left.-\left(\epsilon_{z 32}+\epsilon_{y 32}+\epsilon_{\Delta T}\right)-\left(\epsilon_{z 42}+\epsilon_{y 42}+\epsilon_{\Delta T}\right)\right] \\
& =\frac{E A}{8}\left[\left(B-M+\epsilon_{\Delta T}\right)+\left(B+M+\epsilon_{\Delta T}\right)\right. \\
& +\left(B+D+\epsilon_{\Delta T}\right)+\left(B-D+\epsilon_{\Delta T}\right) \\
& -\left(-B-N+\epsilon_{\Delta T}\right)-\left(-B+N+\epsilon_{\Delta T}\right) \\
& \left.-\left(-B+G+\epsilon_{\Delta T}\right)-\left(-B-G+\epsilon_{\Delta T}\right)\right] \\
& =\frac{E A}{8}(8 B)=E A B .
\end{aligned}
$$

In the calculations above, $\epsilon_{\Delta T}$ is the generated strain in FBGs due to the temperature variation.

Comparing the results from 3.5 and 3.6 shows that the sensor measures the applied axial force without crosstalk and temperature cross-sensitivity errors. For a pure lateral force applied to the sensor in $Y$ axis, 3.2 can be rewritten as:

$$
F_{y}=\frac{E I}{2 d l}(G-(-G))=\frac{E I}{2 d l}(2 G)=\frac{E I G}{d l} .
$$

If temperature variation, twist and other components of contact force are considered, then 3.2 will be written as: 


$$
\begin{aligned}
& F_{y}=\frac{E I}{2 d l}\left[\left(\epsilon_{z 32}+\epsilon_{y 32}+\epsilon_{\Delta T}\right)-\left(\epsilon_{z 42}+\epsilon_{y 42}+\epsilon_{\Delta T}\right)\right] \\
& =\frac{E I}{2 d l}\left[\left(-B+G+\epsilon_{\Delta T}\right)-\left(-B-G+\epsilon_{\Delta T}\right)\right] \\
& =\frac{E I}{2 d l}(2 G)=\frac{E I G}{d l} .
\end{aligned}
$$

The results of 3.7 and 3.8 are equal which shows the crosstalk and temperature variation cannot cause errors in $F_{y}$ measurement. This can be proved for $F_{x}$ measurement, similarly. For a pure twist applied to the sensor, 3.4 can be written as:

$$
T_{z}=P(4 W-(4 W))=8 P W
$$

Considering twist, temperature variation and all components of the contact force, (3.4) is written as: 


$$
\begin{aligned}
& T_{z}=P\left[\left(\epsilon_{z 13}+\epsilon_{x 13}+\epsilon_{T 13}+\epsilon_{\Delta T}\right)\right. \\
& +\left(\epsilon_{z 23}+\epsilon_{x 23}+\epsilon_{T 23}+\epsilon_{\Delta T}\right)+\left(\epsilon_{z 33}+\epsilon_{y 33}+\epsilon_{T 33}+\epsilon_{\Delta T}\right) \\
& +\left(\epsilon_{z 43}+\epsilon_{y 43}+\epsilon_{T 43}+\epsilon_{\Delta T}\right)-\left(\epsilon_{z 14}+\epsilon_{x 14}+\epsilon_{T 14}+\epsilon_{\Delta T}\right) \\
& -\left(\epsilon_{z 24}+\epsilon_{x 24}+\epsilon_{T 24}+\epsilon_{\Delta T}\right)-\left(\epsilon_{z 34}+\epsilon_{y 34}+\epsilon_{T 34}+\epsilon_{\Delta T}\right) \\
& \left.-\left(\epsilon_{z 44}+\epsilon_{y 44}+\epsilon_{T 44}+\epsilon_{\Delta T}\right)\right]=P\left[\left(-C-R+W+\epsilon_{\Delta T}\right)\right. \\
& +\left(-C+R+W+\epsilon_{\Delta T}\right)+\left(-C+H+W+\epsilon_{\Delta T}\right) \\
& +\left(-C-H+W+\epsilon_{\Delta T}\right)-\left(-C-J-W+\epsilon_{\Delta T}\right) \\
& -\left(-C+J-W+\epsilon_{\Delta T}\right)-\left(-C+K-W+\epsilon_{\Delta T}\right) \\
& \left.-\left(-C-K-W+\epsilon_{\Delta T}\right)\right] \\
& =P(8 W)=8 P W
\end{aligned}
$$

and this is the same result obtained from 3.9. Therefore, the sensing method is robust against the crosstalk and temperature variation errors for measurement of $T_{z}$.

\subsection{Sensing element}

For measurement of the generated strains in the sensing structure, any type of strain gauges can be used. However, fiber optic-based sensor provides the best option due to its advantages over other types of sensors. For example, the diameter of a typical single mode optical fiber is around $125 \mu \mathrm{m}$ which is proper for space-confined applications such as cardiac catheterizations. Moreover, the sixteen required strain gauges can be fabricated using four optical fibers, each including four FBG strain sensors. This fact is because of the configuration of the strain gauges in the de- 
signed sensing structure. There are other advantages of optical fiber-based sensors over other types of sensors including immunity to electromagnetic interference, the capability of multiplexing, large bandwidth, light weight and robustness against environmental parameters [66].

FBG is the periodic change of the refractive index inside the core of optical fiber. FBG functions as an optical filter that reflects a specific wavelength called Bragg wavelength (Figure 3.8). The amount of strain applied on an FBG can be extracted by measuring the shift of the Bragg wavelength.

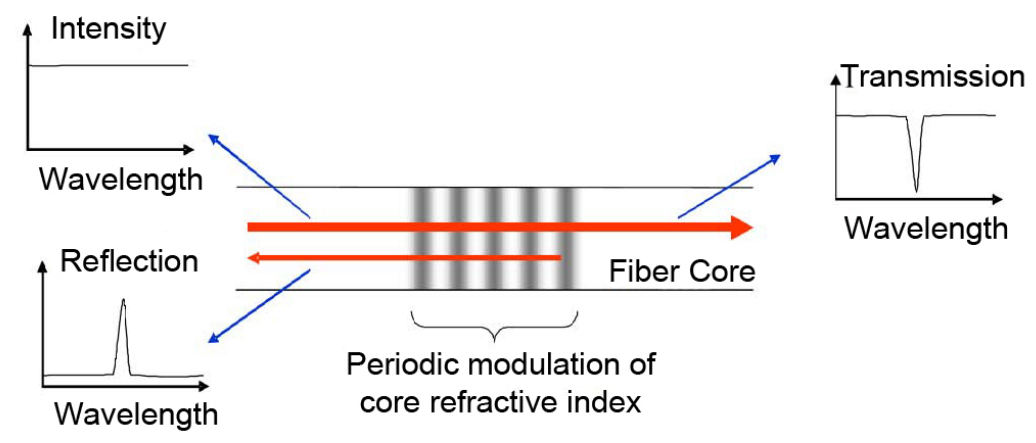

Figure 3.8: FBG reflects a specific wavelength

\subsection{Prototype of the sensor}

The sensing structure of the sensor is fabricated from VisiJet EX200 material using 3D inkjet printing process (ProJet HD 3000, 3D MultiJet printing technique). Material properties of this polymer are listed in Table 3.3. High HDT of this material (56 $\left.{ }^{\circ} \mathrm{C}\right)$ makes it a proper candidate for application in cardiac ablation catheters. HDT indicates the upper limit of safe operation temperature for structures fabricated from polymeric materials [67]. The maximum temperature that can be generated in ablation surgery is $50{ }^{\circ} \mathrm{C}$. However, the maximum applied force to the sensor during the ablation surgery is $0.4 \mathrm{~N}$ which generates the stress of $0.95 \mathrm{MPa}$ in the sensing 
structure. Although this generated stress is larger than the applied stress in HDT test of the material, the experiments performed for thermal insensitivity analysis of the sensor shows that the sensing structure can bear $50{ }^{\circ} \mathrm{C}$ and measure the applied force with high accuracy.

VisiJet EX200 is a bio-compatible material that has passed the USP Class VI tests [68]. This material can be used in surgical instruments and medical devices which are in direct contact with human body.

Figure 3.9 shows the fabricated sensing structure.

Table 3.3: Sensing Structure Material Properties

\begin{tabular}{|l|l|}
\hline Property & Value \\
\hline \hline Density & $1.02 \mathrm{~g} / \mathrm{cm}^{3}$ \\
\hline Tensile Strength & $42.4 \mathrm{MPa}$ \\
\hline Tensile Modulus & $1463 \mathrm{MPa}$ \\
\hline Heat Distortion Temperature at $0.45 \mathrm{MPa}$ & $56{ }^{\circ} \mathrm{C}$ \\
\hline Poisson's Ratio & 0.42 \\
\hline
\end{tabular}

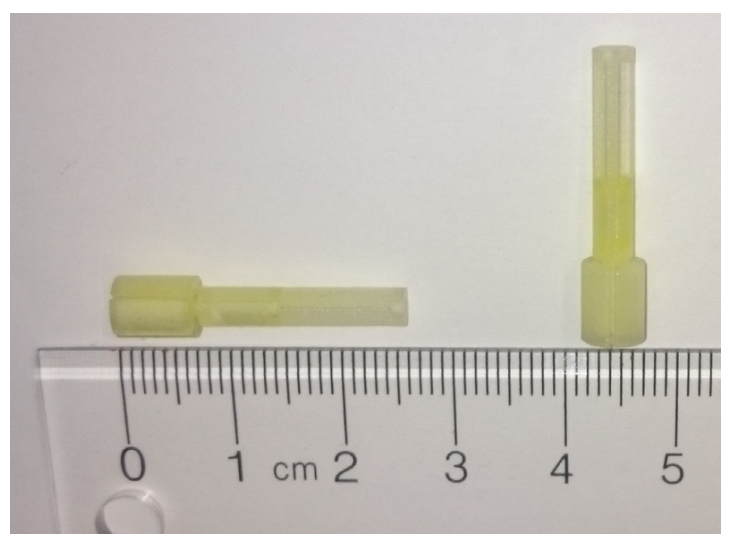

Figure 3.9: Fabricated sensing structure

There are four eccentric holes in the fabricated sensing structure for embedding four optical fibers. Each optical fiber includes four FBGs. The sixteen FBGs measure the generated strains in the sensing structure. 
Because of the symmetry in this sensor for the measurement of lateral forces in $X$ and $Y$ axes, only two optical fibers are used in the experiments to analyze the response of the sensor. Optical fiber 1 includes four FBGs, namely $\mathrm{FBG}_{11}, \mathrm{FBG}_{12}$, $\mathrm{FBG}_{13}$ and $\mathrm{FBG}_{14}$ to measure strains $\epsilon_{11}, \epsilon_{12}, \epsilon_{13}$ and $\epsilon_{14}$, respectively. Optical fiber 2 contains four FBGs, namely $\mathrm{FBG}_{21}, \mathrm{FBG}_{22}, \mathrm{FBG}_{23}$ and $\mathrm{FBG}_{24}$ to measure strains $\epsilon_{21}, \epsilon_{22}, \epsilon_{23}$ and $\epsilon_{24}$, respectively. Table 3.4 provides the Bragg wavelength, reflectivity and bandwidth of FBGs for Fiber 1 and Fiber 2. The length of each FBG is $3 \mathrm{~mm}$. The assembled sensor including embedded optical fibers and sheath of a cardiac catheter is shown in Figure 3.10.

Table 3.4: Bragg Wavelength, Bandwidth and Reflectivity of FBGs in Fiber 1 and Fiber 2

\begin{tabular}{|c|c|c|c|}
\hline & Bragg Wavelength (nm) & Bandwidth (nm) & Reflectivity (\%) \\
\hline \hline $\mathrm{FBG}_{11}$ & 1544.725 & 0.650 & $22<\mathrm{R}<23$ \\
\hline $\mathrm{FBG}_{12}$ & 1539.950 & 0.650 & $22<\mathrm{R}<23$ \\
\hline $\mathrm{FBG}_{13}$ & 1535.350 & 0.638 & $23<\mathrm{R}<24$ \\
\hline $\mathrm{FBG}_{14}$ & 1530.650 & 0.630 & $23<\mathrm{R}<24$ \\
\hline $\mathrm{FBG}_{21}$ & 1544.695 & 0.637 & $23<\mathrm{R}<24$ \\
\hline $\mathrm{FBG}_{22}$ & 1540.016 & 0.650 & $23<\mathrm{R}<24$ \\
\hline $\mathrm{FBG}_{23}$ & 1535.338 & 0.663 & $23<\mathrm{R}<24$ \\
\hline $\mathrm{FBG}_{24}$ & 1530.682 & 0.650 & $25<\mathrm{R}<26$ \\
\hline
\end{tabular}

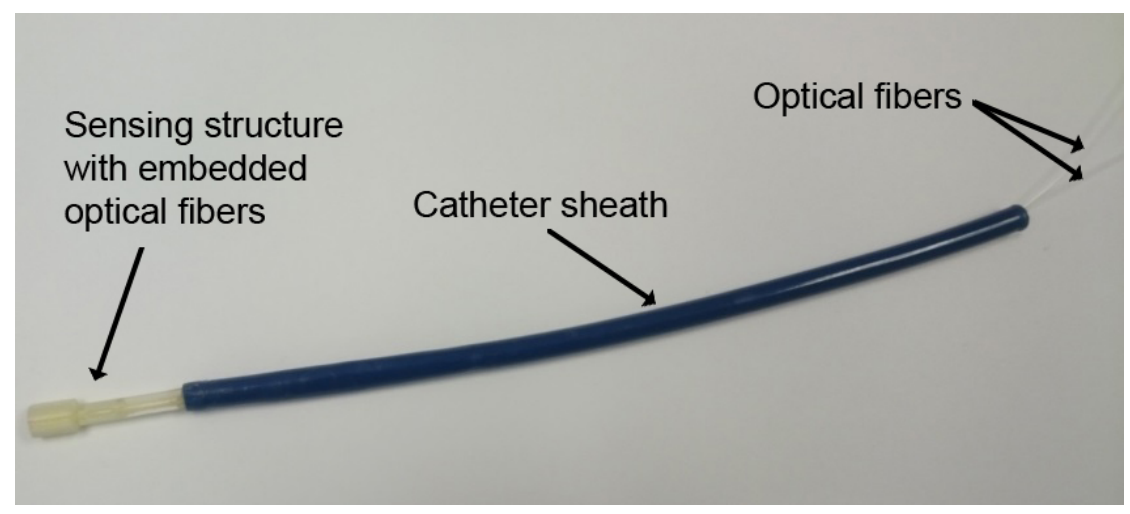

Figure 3.10: The assembled force/torque sensor that includes the sensing structure, optical fibers and catheter sheath. 


\subsection{Experimental results and discussion}

\subsubsection{Static response of the sensor}

In order to calibrate the designed force and torque sensor, a Nano-17 F/T sensor (ATI Industrial Automation, Apex, NC) is used to measure the applied axial and lateral forces. The measurement ranges of ATI sensor for applied force and torque are $12 \mathrm{~N}$ and $120 \mathrm{Nmm}$, respectively. This sensor has measurement resolutions of $0.003 \mathrm{~N}$ and $0.015 \mathrm{Nmm}$ for applied force and torque, respectively [69].

While the fabricated sensor is held with a clamp, the ATI sensor applies force to the tip of the fabricated sensor using a linear stage (Optikon, Kitchener, Canada). The clamp and linear stage are both fixed on an experimental anti-vibration table to avoid any undesired movement of the fabricated sensor and ATI sensor.

Figure 3.11 (a) shows the experimental setup for applying axial force to the fabricated sensor. The axial force is applied to the sensor from 0 to $1 \mathrm{~N}$ in increments of $0.05 \mathrm{~N}$. In all steps of this experiment, lateral components of the contact force were less than $3 \%$ of the applied axial force.

For applying lateral forces to the fabricated sensor, the configuration of the experimental setup is changed slightly as it can be seen in Figure 3.11 (b). The lateral force is applied to the tip of the fabricated sensor in the range of 0 to $0.7 \mathrm{~N}$ by step of $0.05 \mathrm{~N}$. In all steps of this experiment, axial component of the contact force was less than $3 \%$ of the applied lateral force.

The optical fibers are connected to I-MON 512 E spectrometer (Ibsen Photonics, Farum, Denmark) for measuring the Bragg wavelength shifts of the FBGs. This optical spectrometer measures the wavelength shift of the FBG peak to $<0.5 \mathrm{pm}$ with a measurement frequency of $970 \mathrm{~Hz}$ which is sufficient to eliminate environmental 
disturbances.

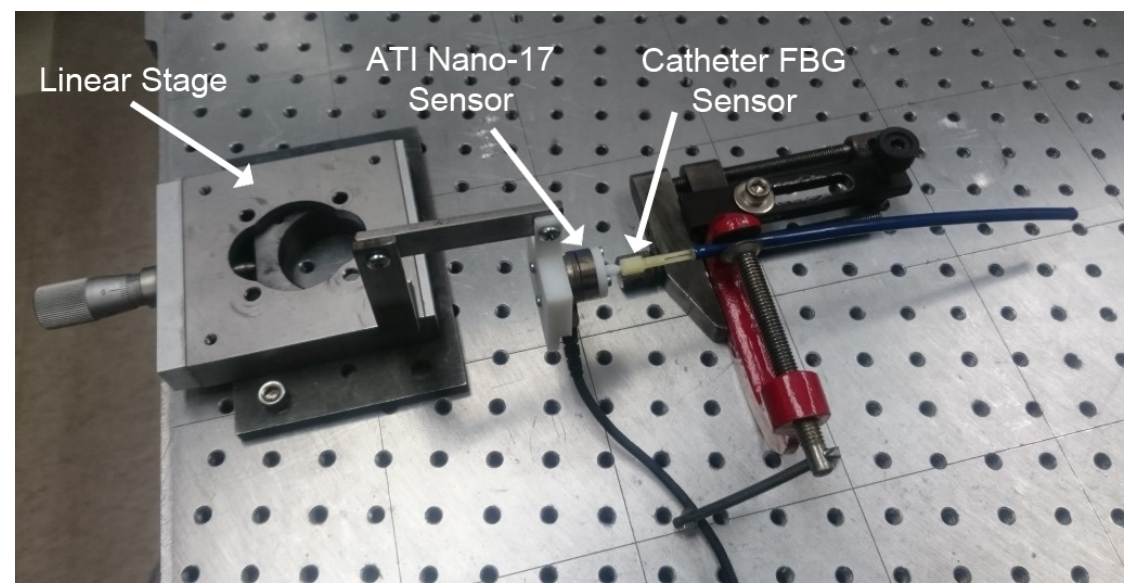

(a)

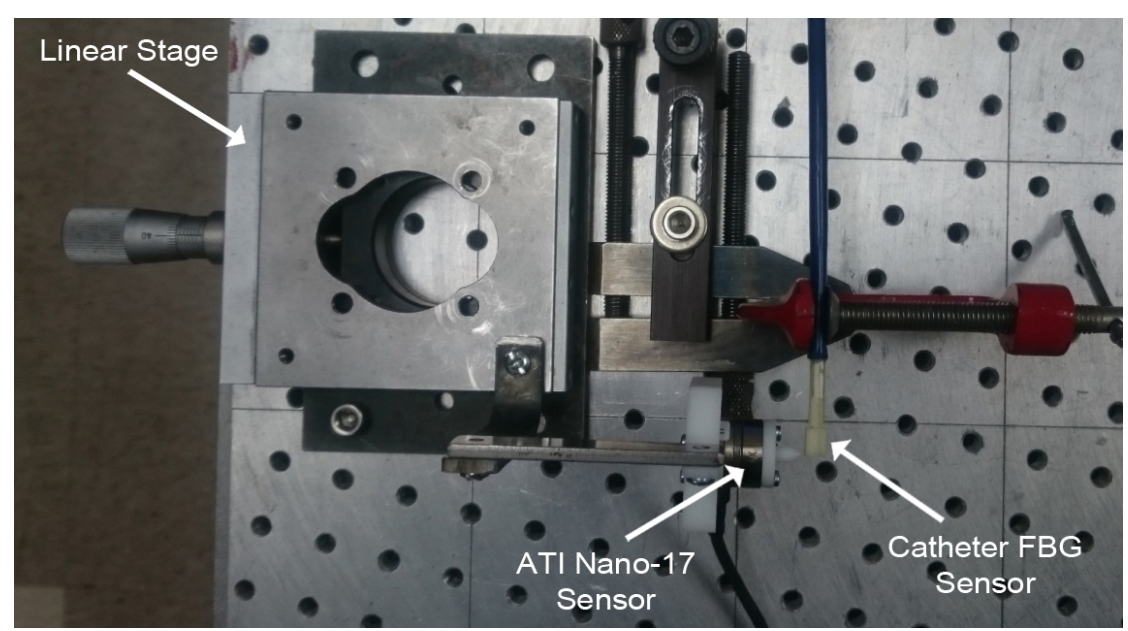

(b)

Figure 3.11: Experimental set-ups for (a) applying axial forces and (b) applying lateral forces.

Figure 3.12 (a) and (b) show the positive shift of Bragg wavelength for $\mathrm{FBG}_{11}$ and the negative shift of Bragg wavelength for $\mathrm{FBG}_{12}$ due to the applied axial force. It can be seen that the measured strains are positive (tensile) and negative (compressive) for $\mathrm{FBG}_{11}$ and $\mathrm{FBG}_{12}$, respectively. As shown in Figure 3.12 (c) and (d), $\mathrm{FBG}_{21}$ and $\mathrm{FBG}_{22}$ experience strains with opposite signs under applied axial force. Experimental results for Bragg wavelength shifts of $\mathrm{FBG}_{12}$ and $\mathrm{FBG}_{22}$ due to the applied lateral forces in the negative direction of $X$ axis are provided in Figure 3.12 
(e) and (f). As shown in this figure, the shift of Bragg wavelength due to the applied lateral force is positive for $\mathrm{FBG}_{12}$ (tensile mode) while the corresponding shift is negative for $\mathrm{FBG}_{22}$ (compressive mode).

In order to apply the torque $\left(T_{z}\right)$ to the fabricated sensor, its distal end is gripped by ATI sensor. A rotational stage that holds ATI sensor is used to generate the torque in the fabricated sensor. This experimental setup can be seen in Figure 3.13. The range of the applied torque is from 0 to $1.5 \mathrm{Nmm}$ by step of $0.1 \mathrm{Nmm}$. The experimental results for the applied torque is shown in Figure $3.12(\mathrm{~g})$ and (h). The shift of Bragg wavelength in $\mathrm{FBG}_{13}$ is negative because of the generated compressive strain, while $\mathrm{FBG}_{14}$ experiences positive Bragg wavelength shift due to the generated tensile strain. Experiments for obtaining the static response of the sensor are repeated seven times for each step, and the mean values are extracted for sketching the plots of Figure 3.12. The maximum amount of Standard Deviation (SD) for each set of experimental data is provided in Table 3.5. The SD of the results for the applied lateral force are higher compared to axial force and twist. The reason is the high sensitivity of the sensor to lateral force compared to axial force and twist. Consequently, any systematic error causes larger deviation of measured Bragg wavelength for lateral forces. 


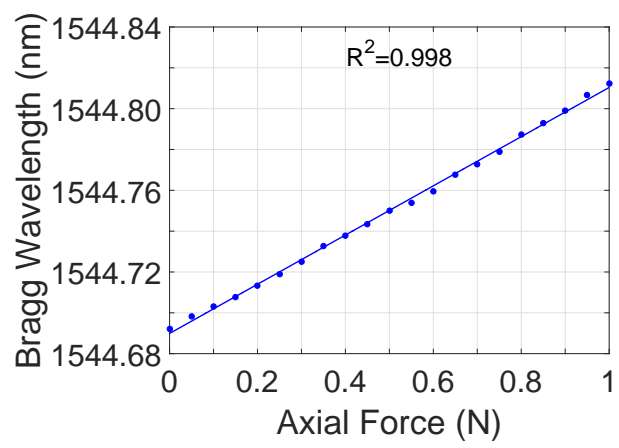

(a)

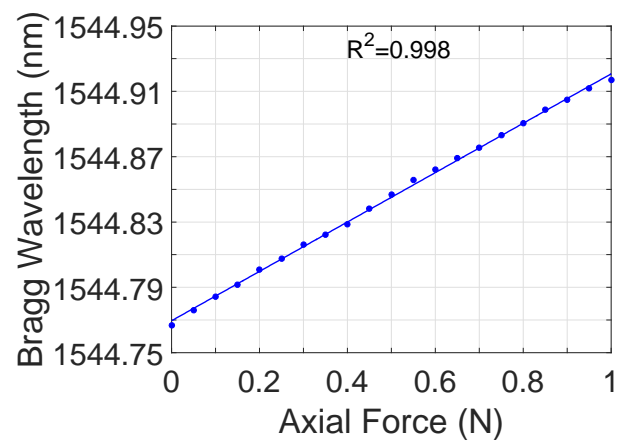

(c)

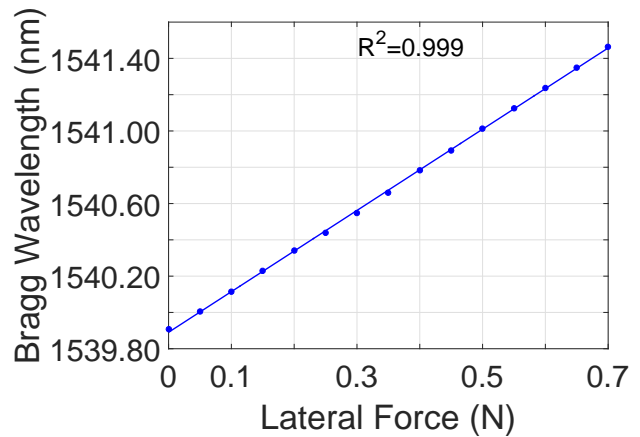

(e)

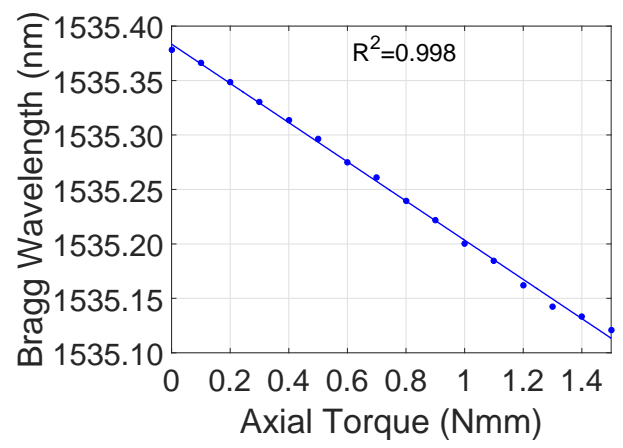

(g)

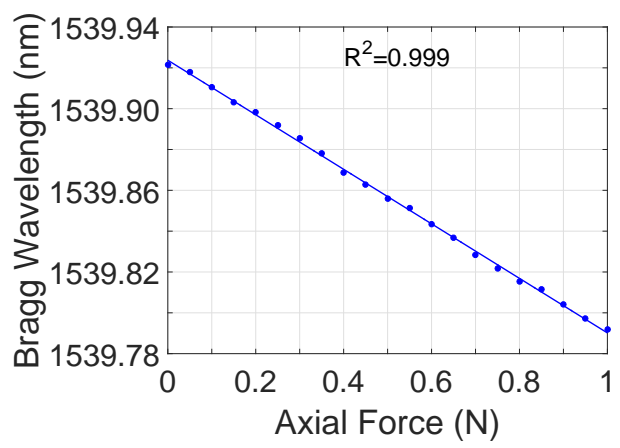

(b)

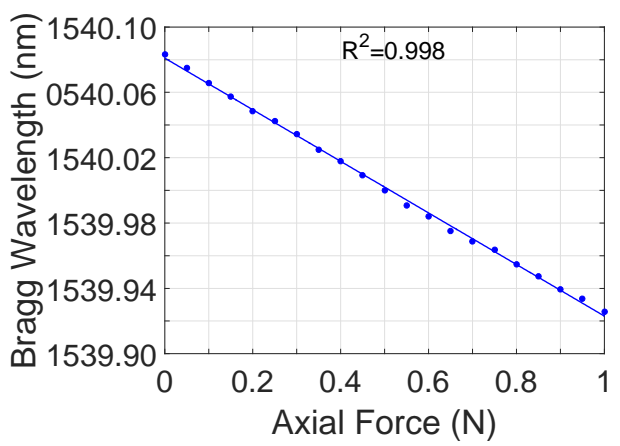

(d)

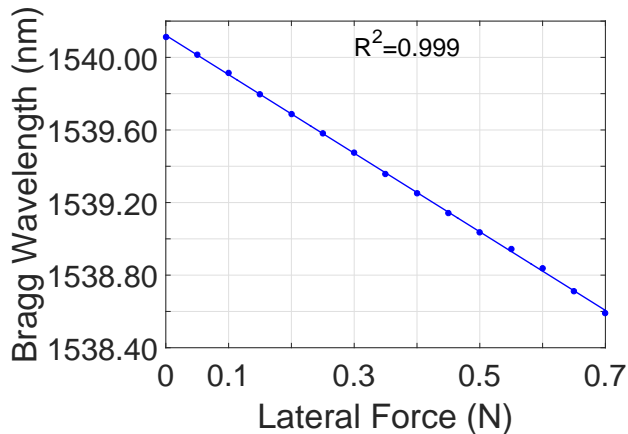

(f)

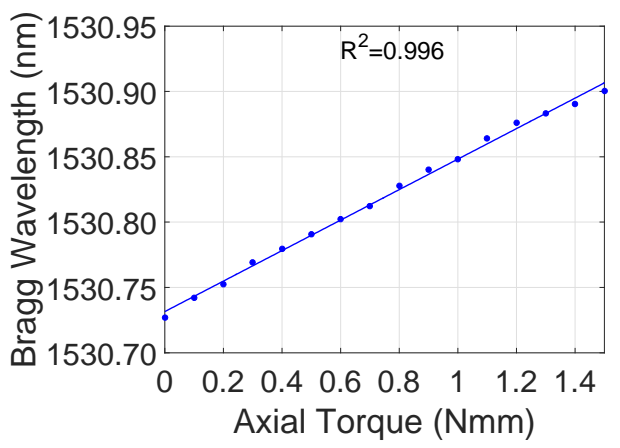

(h)

Figure 3.12: Bragg wavelength shift of FBGs due to the axial force, lateral force and torque

Bragg wavelength shift of (a) $\mathrm{FBG}_{11}$ due to the axial force $F_{z}$, (b) $\mathrm{FBG}_{12}$ due to the axial force $F_{z}$, (c) $\mathrm{FBG}_{21}$ due to the axial force $F_{z}$, (d) $\mathrm{FBG}_{22}$ due to the axial force $F_{z}$, (e) $\mathrm{FBG}_{12}$ due to the lateral force $F_{x}$, (f) $\mathrm{FBG}_{22}$ due to the lateral force $F_{x}$, (g) $\mathrm{FBG}_{13}$ due to the torque $T_{z}$, (h) $\mathrm{FBG}_{14}$ glue to the torque $T_{z}$. 


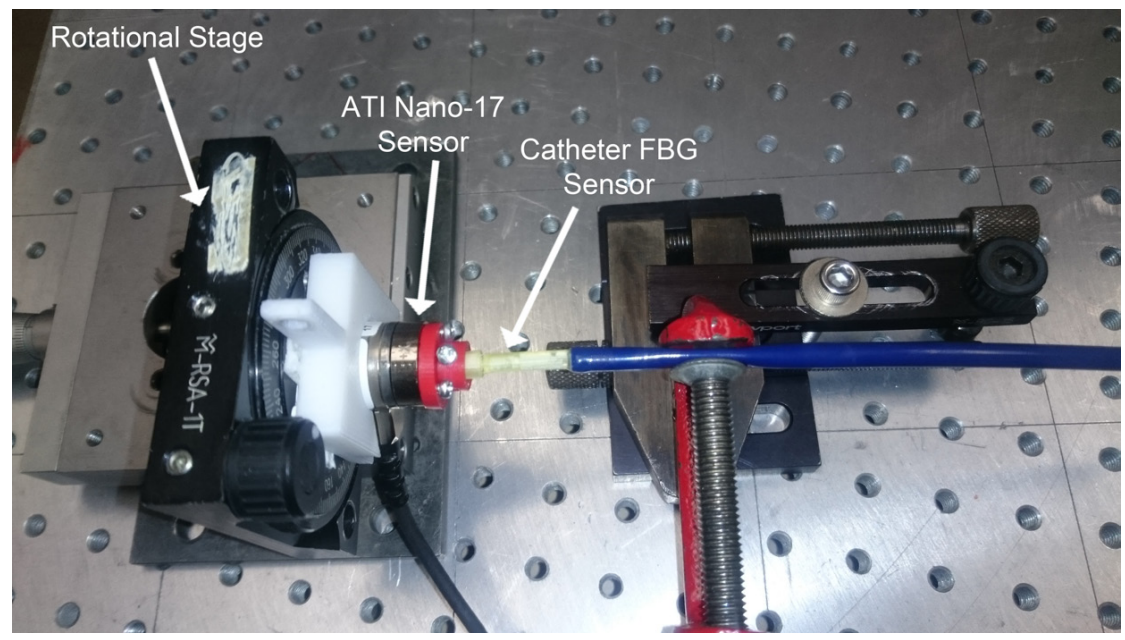

Figure 3.13: Experimental setup for applying torque.

Table 3.5: Maximum SD and Sensitivity for Experimental Results

\begin{tabular}{|l|c|c|}
\hline Experimental Result & Maximum SD & Sensitivity \\
\hline \hline $\mathrm{FBG}_{11}$ under axial force & $0.0021 \mathrm{~nm}$ & $+120 \mathrm{pm} / \mathrm{N}$ \\
\hline $\mathrm{FBG}_{12}$ under axial force & $0.0011 \mathrm{~nm}$ & $-129 \mathrm{pm} / \mathrm{N}$ \\
\hline $\mathrm{FBG}_{21}$ under axial force & $0.0015 \mathrm{~nm}$ & $+150 \mathrm{pm} / \mathrm{N}$ \\
\hline $\mathrm{FBG}_{22}$ under axial force & $0.0012 \mathrm{~nm}$ & $-157 \mathrm{pm} / \mathrm{N}$ \\
\hline $\mathrm{FBG}_{12}$ under lateral force & $0.0125 \mathrm{~nm}$ & $+2225 \mathrm{pm} / \mathrm{N}$ \\
\hline $\mathrm{FBG}_{22}$ under lateral force & $0.0173 \mathrm{~nm}$ & $-2175 \mathrm{pm} / \mathrm{N}$ \\
\hline $\mathrm{FBG}_{13}$ under applied torque & $0.0036 \mathrm{~nm}$ & $-171 \mathrm{pm} / \mathrm{Nmm}$ \\
\hline $\mathrm{FBG}_{14}$ under applied torque & $0.0044 \mathrm{~nm}$ & $+115 \mathrm{pm} / \mathrm{Nmm}$ \\
\hline
\end{tabular}

The experimental results show that the response of the sensor is highly linear to all components of the applied forces and torques. The linearity error for all the achieved results is less than 1\%. Furthermore, FBGs provide high sensitivity of measurement for the sensor. Loading-unloading processes are repeated to evaluate the repeatability of the sensor. The calculated hysteresis ratio based on the maximum deviation from loading-unloading curves is $\mathrm{h}=0.019(1.9 \%)$. 


\subsubsection{Dynamic response of the sensor}

Because of the dynamic movement of the heart during the cardiac cycle, the embedded sensor at the tip of the ablation catheter experiences a dynamic force. Therefore, the response of the designed and fabricated sensor to dynamic force with the frequency of 1-2 Hz (heart frequency) is an important parameter for testing [65]. For applying the dynamic axial force to the fabricated sensor, a Mindstorms NXT 2.0 (LEGO) system is used. As shown in Figure 3.14, the DC motor of the NXT is fixed on the experimental desk, and its shaft is attached to the knob of the linear stage using two gears.

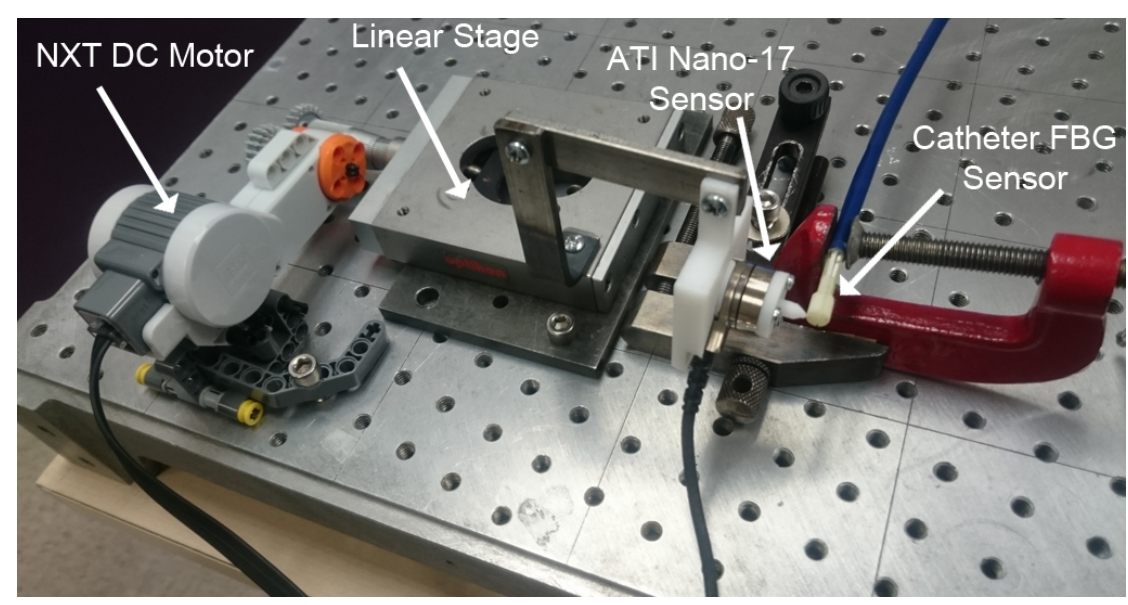

Figure 3.14: Experimental setup for applying dynamic force

The speed and timing of the rotation for the motor are controlled by a program written in RobotC software and downloaded into the ARM7TDMI-core Atmel microcontroller (AT91SAM7S256) of the NXT. Two different input force signals are generated with using this experimental setup. The first type of the input signal is a square wave with the frequency of $0.5 \mathrm{~Hz}$ and the second input signal is a sinusoidal wave with the frequency of $1.5 \mathrm{~Hz}$. The response of the fabricated sensor to the applied axial force signal with the sinusoidal wave and lateral force signal with square wave are shown in Figure 3.15. The calculated coefficient of determination 
$\left(\mathrm{R}^{2}\right)$ for the square wave is 0.912 with root mean square (rms) error of $0.029 \mathrm{~N}$. The coefficient of determination $\left(\mathrm{R}^{2}\right)$ for the sinusoidal wave equals 0.920 with the rms error of $0.026 \mathrm{~N}$. The results show satisfactory dynamic responses.

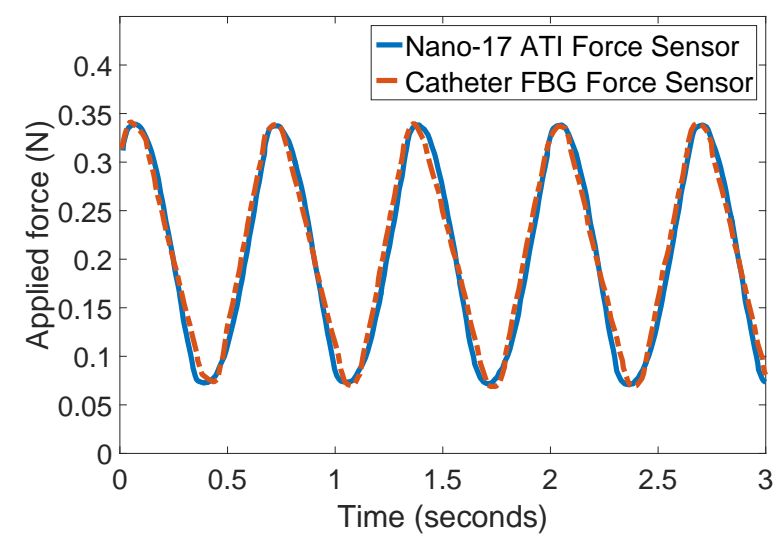

(a)

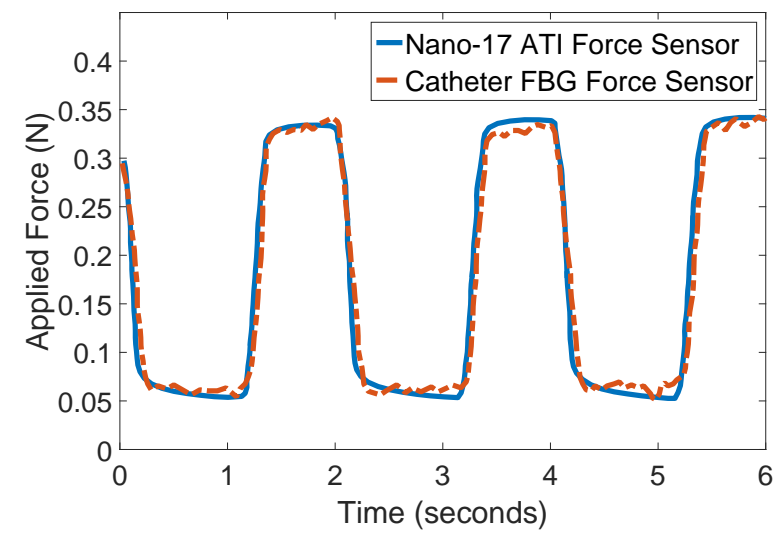

(b)

Figure 3.15: Response of the fabricated sensor to the applied dynamic (a) axial force with sinusoidal wave and (b) lateral force with square wave.

\subsubsection{Temperature insensitivity of the sensor}

For analyzing the response of the sensor in environments with temperature variation, a heating plate is located near the sensing structure of the sensor to change the surrounding temperature and measurements are performed while applying different forces and torques to the sensor. The related experimental setup is shown in 
Figure 3.16.

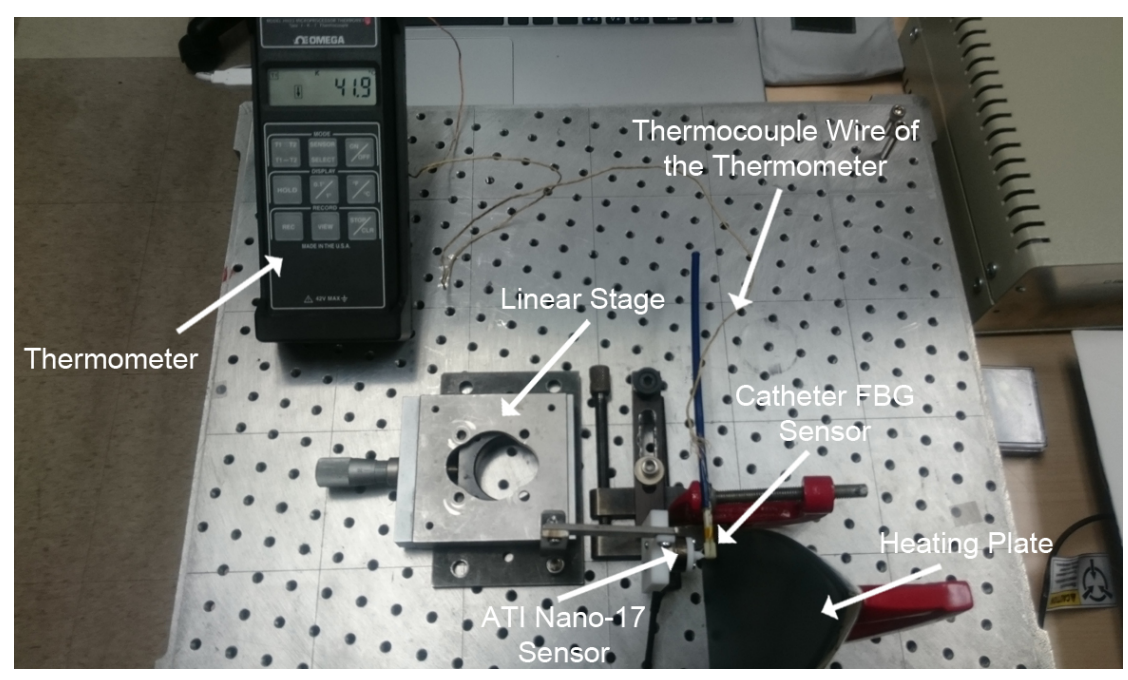

Figure 3.16: Experimental setup for thermal sensitivity analysis of the sensor

The temperature around the fabricated sensor is measured with HH23 digital thermometer (OMEGA Engineering, Stamford, Connecticut) which has measurement resolution of $0.1{ }^{\circ} \mathrm{C}$. The thermocouple wire of the thermometer is attached to the tip of the fabricated force/torque sensor. The temperature around the fabricated sensor is changed from $26{ }^{\circ} \mathrm{C}$ to $50{ }^{\circ} \mathrm{C}$ with steps of $4{ }^{\circ} \mathrm{C}$ and Bragg wavelength of the FBGs were measured at different temperatures. Shifts of Bragg wavelengths for the FBGs (Figure 3.17) show that each FBG experiences temperature variation. Consequently, there are errors in the measured forces and twist using Bragg wavelength shift of single FBG. Figure 3.17 shows the measured applied axial and lateral forces versus temperature using only $\mathrm{FBG}_{12}$ while a constant force of $0.1 \mathrm{~N}$ is applied. This figure also shows the measured twist using only $\mathrm{FBG}_{14}$ while the constant twist of $0.5 \mathrm{Nmm}$ is applied and there is temperature variation. 


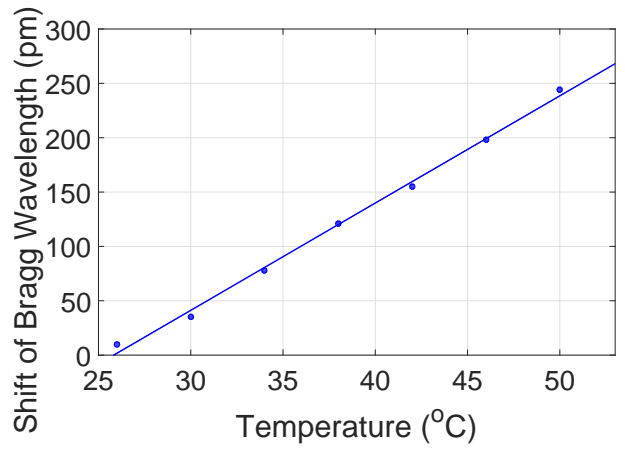

(a)

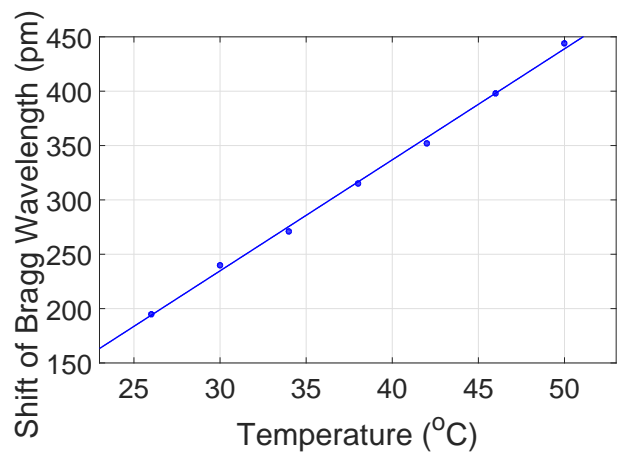

(c)

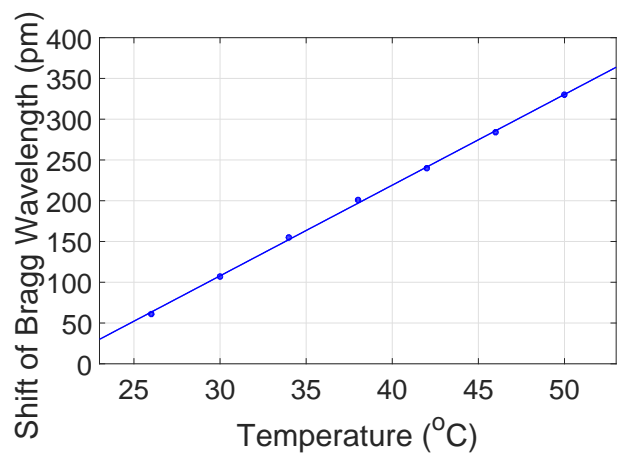

(e)



(b)

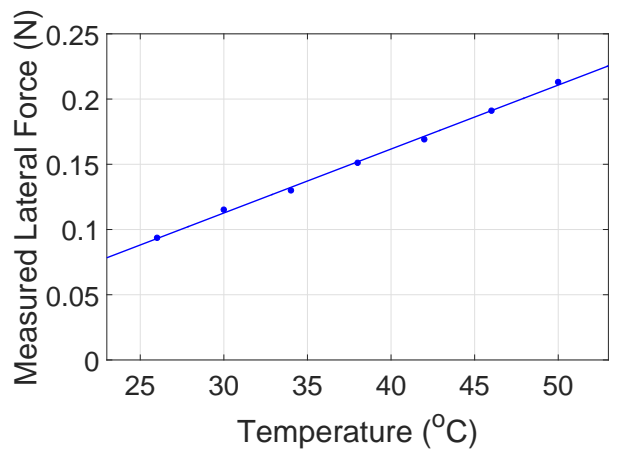

(d)

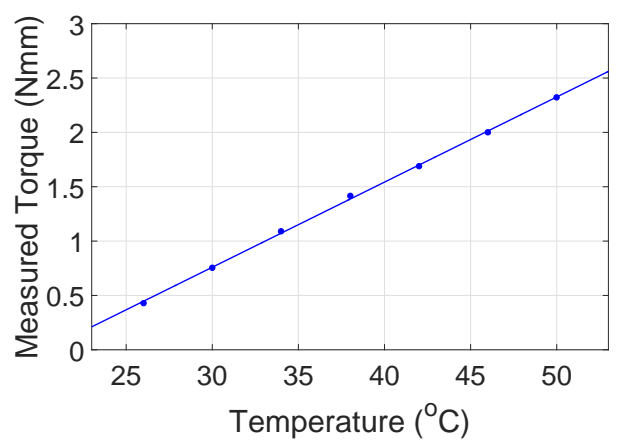

(f)

Figure 3.17: Bragg wavelength of FBGs and calculated forces by the sensor under applied axial force, lateral force and torque with temperature variation.

(a) Bragg wavelength of $\mathrm{FBG}_{12}$ under applied axial force of $0.1 \mathrm{~N}$ and temperature variation. (b) Calculated force based on the Bragg wavelength shift of $\mathrm{FBG}_{12}$ under applied axial force of $0.1 \mathrm{~N}$ and temperature variation. (c) Bragg wavelength of $\mathrm{FBG}_{12}$ under applied lateral force of $0.1 \mathrm{~N}$ and temperature variation. (d) Calculated force based on the Bragg wavelength shift of $\mathrm{FBG}_{12}$ under applied lateral force of $0.1 \mathrm{~N}$ and temperature variation. (e) Bragg wavelength of $\mathrm{FBG}_{14}$ under applied torque of $0.5 \mathrm{Nmm}$ and temperature variation. (f) Calculated torque based on the Bragg wavelength shift of $\mathrm{FBG}_{14}$ under applied torque of $0.5 \mathrm{Nmm}$ and temperature variation. 
The experimental results for measurement of applied forces and torques by the fabricated sensor based on differential measurement method are depicted in Figure 3.18. This figure shows that there is a subtle change in the measured force and torque with temperature variations caused by systematic errors. The main source of systematic error could be temperature variation during readings of FBG Bragg wavelength shifts.

The experimental results show that the thermal cross-sensitivity is significantly decreased by using differential measurement method. The thermal cross-sensitivity error for measurement of applied axial force, lateral force and twist are decreased from $0.09 \mathrm{~N} /{ }^{\circ} \mathrm{C}$ to $2 \times 10^{-4} \mathrm{~N} /{ }^{\circ} \mathrm{C}, 0.005 \mathrm{~N} /{ }^{\circ} \mathrm{C}$ to $6.6 \times 10^{-5} \mathrm{~N} /{ }^{\circ} \mathrm{C}$, and $0.08 \mathrm{Nmm} /{ }^{\circ} \mathrm{C}$ to $0.001 \mathrm{Nmm} /{ }^{\circ} \mathrm{C}$, respectively. Considering the required accuracy for the desirable force sensor which is $0.003 \mathrm{~N}$ ( $1 \%$ of the measured range), the obtained results indicate the high performance of the designed force sensor in the environment with temperature variation.

It is noteworthy to mention that the full-scale accuracy of ATI Nano-17 force/torque sensor is $0.1 \%$ at $22{ }^{\circ} \mathrm{C}$. This accuracy for $\pm 25{ }^{\circ} \mathrm{C}$ deviation from $22{ }^{\circ} \mathrm{C}$ is $0.5 \%$. Therefore, the measured forces and torques by ATI sensor is accurate for the range of temperature variation in this experiment. 
3.8 Experimental results and discussion

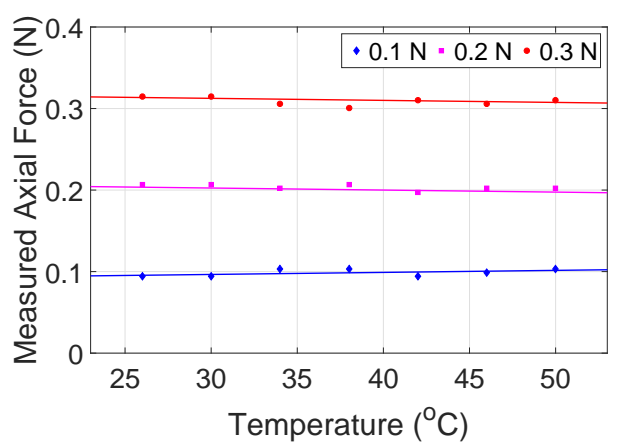

(a)

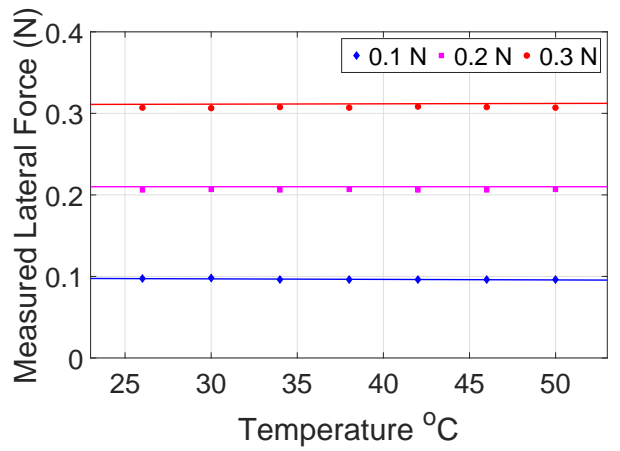

(b)

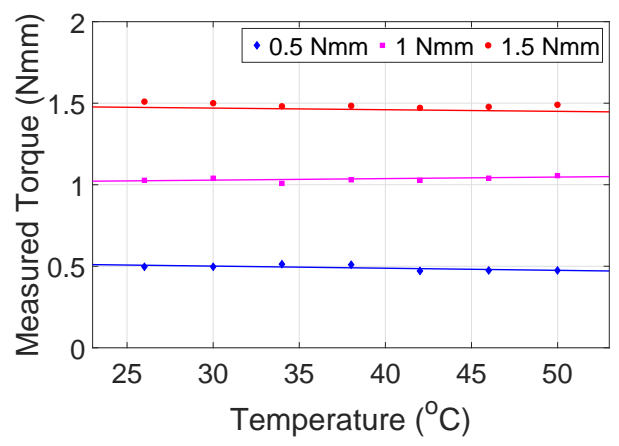

(c)

Figure 3.18: Measured applied forces/torque by fabricated sensor at different temperatures. 


\subsection{Summary}

\subsection{Summary}

In this chapter of the thesis, a force/torque sensor for cardiac catheters was presented $^{1,2}$. The characteristics of the fabricated sensor are provided in Table 3.6. Accuracy error of the sensor is calculated from the maximum SD divided by the maximum value of the measured force and torque.

Table 3.6: Characteristics of the Fabricated Force Sensor

\begin{tabular}{|c|c|}
\hline Parameter & Value \\
\hline \hline Sensor size & $10-15 \mathrm{Fr}$ \\
\hline Measurement range & $0-0.7 \mathrm{~N}$ \\
\hline Sensitivity & $120 \mathrm{pm} / \mathrm{N}, 115 \mathrm{pm} / \mathrm{Nmm}$ \\
\hline Accuracy & $<3 \%$ error \\
\hline Resolution & $>05 \mathrm{~N}, 0.1 \mathrm{Nmm}$ \\
\hline Repeatability & $>92 \%$ \\
\hline Bandwidth & Beating heart frequency $(1-2 \mathrm{~Hz})$ \\
\hline Linearity & Highly linear $(<1 \%$ error $)$ \\
\hline Hysteresis $(<2 \%)$ \\
\hline Thermal cross sensitivity error & $<0.001 \mathrm{~N} /{ }^{\circ} \mathrm{C}$ \\
\hline MRI compatibility & Yes \\
\hline Blood contamination & Resistant to body fluids \\
\hline Cost of prototype & $\$ 400-\$ 600$ \\
\hline
\end{tabular}

The differential type of measurement used in this sensor eliminates the thermal cross-sensitivity error as well as the crosstalk between the measurement channels. The sensing structure was evaluated through the FEM simulations and large-scale prototyping. Various experiments were performed on the actual-scale prototype of the sensor to evaluate the statistic response, dynamic response and temperature insensitivity of the sensor.

\footnotetext{
${ }^{1}$ The patent for this force/torque sensor has been filed and published: F. Janabi-Sharifi, A. N. Cheema and A. Taghipour, "Temperature invariant force and torque sensor assemblies" Patent WO2016149819 A1, Sep. 29, 2016.

${ }^{2}$ The paper for this force/torque sensor has been submitted to IEEE/ASME Transactions on Mechatronics: A. Taghipour, A. N. Cheema, X. Gu and F. Janabi-Sharifi, "Temperature independent tri-axial force and torque sensor for minimally invasive interventions," submitted to IEEE/ASME Transactions on Mechatronics.
} 


\section{Conclusion and future directions}

\subsection{Conclusion}

In this thesis, a novel catheter-based tunable device for percutaneous treatment of MR disease was presented. The device was designed to push each $\mathrm{P}$ point of the posterior leaflet independently while the force applied on LCx artery is minimized. The design procedure was based on CT scan analysis of 204 patients to extract the dimensions of CS and its geometrical parameters with respect to MA. Moreover, the required amount of force applied to each $\mathrm{P}$ point of posterior leaflet for generating effective displacement in MA was extracted from performing experiments on pig hearts. The extracted value of forces were used in the FEM simulations for stress analysis of the device. The presented device is the first catheter-based tunable device in the world for percutaneous treatment of MR. The previous devices cannot be implanted for $55 \%$ of the patients due to the probability of LCx compression because of being located between CS and MA. The main problem of the previous devices, which were designed for MR treatment from CS, is the possibility of LCx artery compression. This fact arises from the lack of control on the location of force applied to MA. Using the previous devices, the surgeon does not have control on the location and amount of force applied to MA. The designed device in this thesis solves this problem and introduces a new concept of percutaneous treatment of MR 


\subsection{Conclusion}

in which surgeons can apply different forces to different points of MA such that the LCx artery compression is minimized.

In addition, the designing and testing a temperature insensitive force/torque sensor for cardiac catheters were performed. It was shown theoretically that the measured force and torque by this sensor are not affected by temperature variations, and the crosstalk effect between channels is canceled. FEM simulations were performed to evaluate and develop the sensing structure of this sensor. The large-scale prototype of the sensor was fabricated, and the experiments were conducted to evaluate the sensing structure under different applied forces and torques. Finally, the actualscale prototype of the sensor was fabricated for testing. Various experiments were performed to evaluate the statistic response, dynamic response and temperature insensitivity of the sensor. This designed and fabricated sensor is the first force/torque sensor in the world that can measure all components of the applied force and torque to the distal end of cardiac catheters without temperature cross-sensitivity. This sensor can be used in different cardiac catheters for obtaining the contact information between the catheter and body organs. This sensor also helps development of surgical robotic systems by providing temperature insensitive measurement of the contact force.

In the end, the contributions of this thesis can be summarized as:

- Designing the first catheter-based tunable device in the world for percutaneous treatment of MR with capability of modifying the amount of applied force on the MA and minimizing the compression on the LCx artery

- Comprehensive study of CS size and geometry with respect to the MA by CT scan images analysis of 204 patients

- Extracting the amount of required force to generate the desired value of the displacement in the MA at $\mathrm{P}$ points of the posterior leaflet of MV 
- Designing, large-scale prototyping and testing a novel engagement mechanism for engagement steerable catheter with implanted cardiac device

- Designing, prototyping and testing a novel 6DOF temperature insensitive force/torque sensor for cardiac catheters

\subsection{Future directions}

The contributions made in this thesis can be the foundation for other future projects. The potential future directions from this thesis are described in the following subsections.

\subsubsection{Catheter-based device for percutaneous treatment of MR}

One possible future work on the designed catheter-based device would be the actualsize prototyping and performing experiments in the lab to evaluate the performance of the device. Then the animal tests would be performed to ensure that the device is safe to be implanted and is effective to improve the MR grade.

Another possible future direction for the designed catheter-based device is integrating shape sensors with the device for real-time detection of the MA shape.

Moreover, the experiment for measuring the required force applied to MA was performed on a static heart. Therefore, as a future work, the experiment can be performed on a beating heart to extract the dynamic force applied to MA.

Another future work on the designed catheter-based device would be performing experiments of applying force to MA from more than one point simultaneously to study the effect of multiple applied force on the shape of MA. 


\subsubsection{Force sensor for cardiac catheters}

The performance of the developed temperature insensitive force/torque sensor for cardiac catheters was evaluated for the application of the ablation catheters by performing different experiments. One possible future work on the proposed force/torque sensor is evaluation of the dynamic response of the sensor in higher frequencies (for example $1 \mathrm{kHz}$ ) to extend the applications of the sensor.

Performing animal tests on this sensor can evaluate its performance and reveal the success rate of the design and method of measurement used in this sensor. Therefore, the critical future step in developing this sensor is performing animal tests and using this sensor in the real operational conditions.

It is noteworthy to mention that performing the animal test is an essential step before applying for Health Canada or FDA and commercializing the designed catheterbased device and the force/torque sensor. 


\section{Bibliography}

[1] F. Alessandrini, N. Luciani, C. Marchetti, M. Gaudino, G. Possati, M. Cortrufo et al., "Early results with the minimally invasive thoracotomy for myocardial revascularization," European Journal of Cardio-Thoracic Surgery, vol. 11, no. 6, pp. 1081-1085, 1997.

[2] E. Alderman, K. Andrews, M. Brooks, K. Detre, S. Kelsey, A. Rosen, K. SuttonTyrrell, M. Bourassa, B. Chaitman, D. Faxon et al., "Five-year clinical and functional outcome comparing bypass surgery and angioplasty in patients with multivessel coronary disease-a multicenter randomized trial," Journal of the American Medical Association (JAMA), vol. 277, no. 9, pp. 715-721, 1997.

[3] L. I. Bonchek, D. J. Ullyot, M. Daniel, and J. Ullyot, "Minimally invasive coronary bypass: A dissenting opinion," Circulation, Journal of the American Heart Association, vol. 98, no. 6, pp. 495-497, 1999.

[4] M. L. Eaton and D. L. Kennedy, Innovation in Medical Technology: Ethical Issues and Challenges. Baltimore: The Johns Hopkings University Press, 2007.

[5] F. N. Delling and R. S. Vasan, "Epidemiology and pathophysiology of mitral valve prolapse," Circulation, vol. 129, no. 21, pp. 2158-2170, 2014.

[6] C. Tamburino and G. P. Ussia, Percutaneous Treatment of Left Side Cardiac 
Bibliography

Valves: A Practical Guide for the Interventional Cardiologist. Berlin, Germany: Springer Science \& Business Media, 2012.

[7] K. A. Eagle and R. R. Baliga, Practical cardiology: evaluation and treatment of common cardiovascular disorders. Philadelphia: Lippincott Williams \& Wilkins, 2008.

[8] S. Kar, "Percutaneous transcatheter mitral valve repair," Journal of the American College of Cardiology, vol. 62, no. 12, pp. 1062-1064, 2013.

[9] P. T. Chiam and C. E. Ruiz, "Percutaneous transcatheter mitral valve repair: a classification of the technology," JACC: Cardiovascular Interventions, vol. 4, no. 1, pp. 1-13, 2011.

[10] C. Fucci, L. Sandrelli, A. Pardini, L. Torracca, M. Ferrari, and O. Alfieri, "Improved results with mitral valve repair using new surgical techniques." European Journal of Cardio-Thoracic Surgery, vol. 9, no. 11, pp. 621-627, 1995.

[11] F. Maisano, A. Caldarola, A. Blasio, M. De Bonis, G. La Canna, and O. Alfieri, "Midterm results of edge-to-edge mitral valve repair without annuloplasty," The Journal of Thoracic and Cardiovascular Surgery, vol. 126, no. 6, pp. 1987-1997, 2003.

[12] P. W. Fedak, P. M. McCarthy, and R. O. Bonow, "Evolving concepts and technologies in mitral valve repair," Circulation, vol. 117, no. 7, pp. 963-974, 2008.

[13] J. G. Webb, J. Harnek, B. I. Munt, P. O. Kimblad, M. Chandavimol, C. R. Thompson, J. R. Mayo, and J. O. Solem, "Percutaneous transvenous mitral annuloplasty," Circulation, vol. 113, no. 6, pp. 851-855, 2006.

[14] J. Harnek, J. G. Webb, K.-H. Kuck, C. Tschope, A. Vahanian, C. E. Buller, S. K. James, C. P. Tiefenbacher, and G. W. Stone, "Transcatheter implanta- 
Bibliography

tion of the monarc coronary sinus device for mitral regurgitation: 1-year results from the evolution phase i study (clinical evaluation of the edwards lifesciences percutaneous mitral annuloplasty system for the treatment of mitral regurgitation)," JACC: Cardiovascular Interventions, vol. 4, no. 1, pp. 115-122, 2011.

[15] J. Schofer, T. Siminiak, M. Haude, J. P. Herrman, J. Vainer, J. C. Wu, W. C. Levy, L. Mauri, T. Feldman, R. Y. Kwong et al., "Percutaneous mitral annuloplasty for functional mitral regurgitation," Circulation, vol. 120, no. 4, pp. 326-333, 2009.

[16] N. Rafiee, N. Douk, and J. F. Crittenden, "Coronary sinus approach for repair of mitral valve regurgitation," Patent US 7955 384, Jun. 7, 2011.

[17] S. Sack, P. Kahlert, L. Bilodeau, L. A. Pièrard, P. Lancellotti, V. Legrand, J. Bartunek, M. Vanderheyden, R. Hoffmann, P. Schauerte et al., "Percutaneous transvenous mitral annuloplasty," Circulation: Cardiovascular Interventions, vol. 2, no. 4, pp. 277-284, 2009.

[18] K. Yokoyama, H. Nakagawa, D. C. Shah, H. Lambert, G. Leo, N. Aeby, A. Ikeda, J. V. Pitha, T. Sharma, R. Lazzara et al., "Novel contact force sensor incorporated in irrigated radiofrequency ablation catheter predicts lesion size and incidence of steam pop and thrombusclinical perspective," Circulation: Arrhythmia and Electrophysiology, vol. 1, no. 5, pp. 354-362, 2008.

[19] G. V. Klimovitch and J. W. Sliwa, "System and method for measuring force and torque applied to a catheter electrode tip," Patent US 8864 757, Oct. 21, 2014 .

[20] J. Rosen, M. MacFarlane, C. Richards, B. Hannaford, and M. Sinanan, "Surgeon-tool force/torque signatures-evaluation of surgical skills in minimally 
Bibliography

invasive surgery," in Proceedings of Medicine Meets Virtual Reality, San Francisco, CA, Jan. 1999, pp. 290-296.

[21] P. Kanagaratnam, M. Koa-Wing, D. T. Wallace, A. S. Goldenberg, N. S. Peters, and D. W. Davies, "Experience of robotic catheter ablation in humans using a novel remotely steerable catheter sheath," Journal of Interventional Cardiac Electrophysiology, vol. 21, no. 1, pp. 19-26, 2008.

[22] C. Tom, "Pressure sensor for therapeutic delivery device and method," Patent US 6695 808, Feb. 24, 2004.

[23] J. Peirs, J. Clijnen, D. Reynaerts, H. Van Brussel, P. Herijgers, B. Corteville, and S. Boone, "A micro optical force sensor for force feedback during minimally invasive robotic surgery," Sensors and Actuators A: Physical, vol. 115, no. 2, pp. 447-455, 2004.

[24] N. Aeby and G. Leo, "Catheter having tri-axial force sensor," Patent US 8048 063, Nov. 1, 2011.

[25] P. Polygerinos, P. Puangmali, T. Schaeffter, R. Razavi, L. D. Seneviratne, and K. Althoefer, "Novel miniature mri-compatible fiber-optic force sensor for cardiac catheterization procedures," in IEEE International Conference on Robotics and Automation (ICRA), Anchorage, AK, May 2010, pp. 2598-2603.

[26] P. Polygerinos, L. D. Seneviratne, R. Razavi, T. Schaeffter, and K. Althoefer, "Triaxial catheter-tip force sensor for mri-guided cardiac procedures," IEEE/ASME Transactions on Mechatronics, vol. 18, no. 1, pp. 386-396, 2013.

[27] O. J. Eick, "Temperature controlled radiofrequency ablation," Indian Pacing and Electrophysiology Journal, vol. 2, no. 3, pp. 66-73, 2002.

[28] S. Blumenkranz and D. Larkin, "Force and torque sensing for surgical instruments," Patent US 14/591 826, Jun. 18, 2015. 
[29] J. Arata, S. Terakawa, and H. Fujimoto, "Fiber optic force sensor for medical applications within a backbone-shape structure," Procedia CIRP, vol. 5, pp. 66-69, 2013.

[30] I. Iordachita, Z. Sun, M. Balicki, J. U. Kang, S. J. Phee, J. Handa, P. Gehlbach, and R. Taylor, "A sub-millimetric, $0.25 \mathrm{mn}$ resolution fully integrated fiberoptic force-sensing tool for retinal microsurgery," International Journal of Computer Assisted Radiology and Surgery, vol. 4, no. 4, pp. 383-390, 2009.

[31] Y. Noh, H. Liu, S. Sareh, D. S. Chathuranga, H. Würdemann, K. Rhode, and K. Althoefer, "Image-based optical miniaturized three-axis force sensor for cardiac catheterization," IEEE Sensors Journal, vol. 16, no. 22, pp. 7924-7932, 2016.

[32] L. Xu, M. I. Miller, J. Ge, K. R. Nilsson, Z. T. H. Tse, and M. P. Fok, "Temperature-insensitive fiber-optic contact force sensor for steerable catheters," IEEE Sensors Journal, vol. 16, no. 12, pp. 4771-4775, 2016.

[33] D. Maselli, F. Guarracino, F. Chiaramonti, F. Mangia, G. Borelli, and G. Minzioni, "Percutaneous mitral annuloplasty an anatomic study of human coronary sinus and its relation with mitral valve annulus and coronary arteries," Circulation, vol. 114, no. 5, pp. 377-380, 2006.

[34] R. del Valle-Fernández, V. Jelnin, G. Panagopoulos, and C. E. Ruiz, "Insight into the dynamics of the coronary sinus/great cardiac vein and the mitral annulus implications for percutaneous mitral annuloplasty techniques," Circulation: Cardiovascular Interventions, vol. 2, no. 6, pp. 557-564, 2009.

[35] S. El-Maasarany, C. G. Ferrett, A. Firth, M. Sheppard, and M. Y. Henein, "The coronary sinus conduit function: anatomical study (relationship to adjacent structures)," Europace, vol. 7, no. 5, pp. 475-481, 2005. 
[36] A. Sorgente, Q. A. Truong, C. Conca, J. P. Singh, U. Hoffmann, F. F. Faletra, C. Klersy, R. Bhatia, G. B. Pedrazzini, E. Pasotti et al., "Influence of left atrial and ventricular volumes on the relation between mitral valve annulus and coronary sinus," The American Journal of Cardiology, vol. 102, no. 7, pp. 890-896, 2008.

[37] D. Sahni, A. Randhawa, A. Aggarwal, and M. K. Rohit, "Spatial relationship of coronary sinus-great cardiac vein with adjoining anatomic structures: a key element in predicting the success of percutaneous transvenous mitral annuloplasty." The Journal of Heart Valve Disease, vol. 23, no. 2, pp. 184-192, 2014.

[38] A. Chiribiri, S. Kelle, U. Köhler, L. F. Tops, B. Schnackenburg, R. Bonamini, J. J. Bax, E. Fleck, and E. Nagel, "Magnetic resonance cardiac vein imaging: relation to mitral valve annulus and left circumflex coronary artery," JACC: Cardiovascular Imaging, vol. 1, no. 6, pp. 729-738, 2008.

[39] S. Mao, J. S. Shinbane, M. J. Girsky, J. Child, S. Carson, R. J. Oudiz, and M. J. Budoff, "Coronary venous imaging with electron beam computed tomographic angiography: three-dimensional mapping and relationship with coronary arteries," American Heart Journal, vol. 150, no. 2, pp. 315-322, 2005.

[40] J. S. Shinbane, M. D. Lesh, W. G. Stevenson, T. S. Klitzner, P. D. Natterson, I. Wiener, P. C. Ursell, and L. A. Saxon, "Anatomic and electrophysiologic relation between the coronary sinus and mitral annulus: implications for ablation of left-sided accessory pathways," American Heart Journal, vol. 135, no. 1, pp. 93-98, 1998.

[41] P. Lancellotti, L. Moura, L. A. Pierard, B. A. Popescu, C. Tribouilloy, A. Hagendorff, J.-L. Monin, L. Badano, J. L. Zamorano, R. Sicari et al., "European association of echocardiography recommendations for the assessment of valvular regurgitation. part 2: mitral and tricuspid regurgitation (native valve disease)," 
Bibliography

European Heart Journal-Cardiovascular Imaging, vol. 11, no. 4, pp. 307-332, 2010 .

[42] S. Ray, "The echocardiographic assessment of functional mitral regurgitation," European Heart Journal-Cardiovascular Imaging, vol. 11, no. 10, pp. i11-i17, 2010.

[43] A. P. W. Lee, M. C. Hsiung, I. S. Salgo, F. Fang, J.-M. Xie, Y.-C. Zhang, Q.-S. Lin, J.-L. Looi, S. Wan, R. H. Wong et al., "Quantitative analysis of mitral valve morphology in mitral valve prolapse using real-time three-dimensional echocardiography: importance of annular saddle-shape in pathogenesis of mitral regurgitation," Circulation, vol. 112, pp. 1-23, 2012.

[44] K. Franco and E. Verrier, Advanced Therapy in Cardiac Surgery, ser. Advanced Therapy in Cardiac Surgery. Hamilton, Canada: B.C. Decker, 2003.

[45] M. O. Jensen, H. Jensen, M. Smerup, R. A. Levine, A. P. Yoganathan, H. Nygaard, J. M. Hasenkam, and S. L. Nielsen, "Saddle-shaped mitral valve annuloplasty rings experience lower forces compared with flat rings," Circulation, vol. 118, no. 14, pp. 250-255, 2008.

[46] G. Dwivedi, G. Mahadevan, D. Jimenez, M. Frenneaux, and R. P. Steeds, "Reference values for mitral and tricuspid annular dimensions using two-dimensional echocardiography," Journal of Echo Research and Practice, vol. 1, no. 2, pp. $43-50,2014$.

[47] L. F. Tops, N. R. Van de Veire, J. D. Schuijf, A. de Roos, E. E. van der Wall, M. J. Schalij, and J. J. Bax, "Noninvasive evaluation of coronary sinus anatomy and its relation to the mitral valve annulus implications for percutaneous mitral annuloplasty," Circulation, vol. 115, no. 11, pp. 1426-1432, 2007.

[48] A. Plass, I. Valenta, O. Gaemperli, P. Kaufmann, H. Alkadhi, G. Zund, J. Grü- 
Bibliography

nenfelder, and M. Genoni, "Assessment of coronary sinus anatomy between normal and insufficient mitral valves by multi-slice computertomography for mitral annuloplasty device implantation," European Journal of Cardio-Thoracic Surgery, vol. 33, no. 4, pp. 583-589, 2008.

[49] A. J. Choure, M. J. Garcia, B. Hesse, M. Sevensma, G. Maly, N. L. Greenberg, L. Borzi, S. Ellis, E. M. Tuzcu, and S. R. Kapadia, "In vivo analysis of the anatomical relationship of coronary sinus to mitral annulus and left circumflex coronary artery using cardiac multidetector computed tomography," Journal of the American College of Cardiology, vol. 48, no. 10, pp. 1938-1945, 2006.

[50] E. Lansac, I. Di Centa, N. Al Attar, D. Messika-Zeitoun, R. Raffoul, A. Vahanian, and P. Nataf, "Percutaneous mitral annuloplasty through the coronary sinus: an anatomic point of view," The Journal of Thoracic and Cardiovascular Surgery, vol. 135, no. 2, pp. 376-381, 2008.

[51] E. Topol and R. Califf, Textbook of Cardiovascular Medicine. Philadelphia: Lippincott Williams \& Wilkins, 2007.

[52] E. Kuwahara, M. Izumo, Y. Fukuoka, S. V. Gurudevan, K. Tolstrup, R. J. Siegel, and T. Shiota, "Relationship between jet direction and mitral valve tethering in patients with ischemic mitral regurgitation: Real-time three dimensonal transesophageal echocardiography study," Circulation, vol. 122, no. 21, pp. A19 873-A19 873, 2010.

[53] S. Buchner, F. Poschenrieder, O. W. Hamer, C. Jungbauer, M. Resch, C. Birner, C. Fellner, G. A. Riegger, C. Stroszczynski, B. Djavidani et al., "Direct visualization of regurgitant orifice by $\mathrm{cmr}$ reveals differential asymmetry according to etiology of mitral regurgitation," JACC: Cardiovascular Imaging, vol. 4, no. 10, pp. 1088-1096, 2011. 
[54] N. A. Marsan, J. J. Westenberg, C. Ypenburg, V. Delgado, R. J. van Bommel, S. D. Roes, G. Nucifora, R. J. van der Geest, A. de Roos, J. C. Reiber et al., "Quantification of functional mitral regurgitation by real-time 3d echocardiography," JACC: Cardiovascular Imaging, vol. 2, no. 11, pp. 1245-1252, 2009.

[55] J. Magne, P. Lancellotti, and L. A. Piérard, "Exercise pulmonary hypertension in asymptomatic degenerative mitral regurgitation," Circulation, vol. 122, no. 1, pp. 33-41, 2010.

[56] (Accessed Jun. 2, 2017). [Online]. Available: http://www.microlight.fr/static/ assets/img/datasheet.pdf

[57] H. Rack and J. Qazi, "Titanium alloys for biomedical applications," Materials Science and Engineering: C, vol. 26, no. 8, pp. 1269-1277, 2006.

[58] P. D. Stein and H. N. Sabbah, "Measured turbulence and its effect on thrombus formation," Circulation Research, vol. 35, no. 4, pp. 608-614, 1974.

[59] M. Fogel, Ventricular Function and Blood Flow in Congenital Heart Disease. Hoboken: Wiley, 2008.

[60] W. Mohl, C. Gangl, A. Jusić, T. Aschacher, M. De Jonge, and F. Rattay, "Picso: from myocardial salvage to tissue regeneration," Cardiovascular Revascularization Medicine, vol. 16, no. 1, pp. 36-46, 2015.

[61] W. Ganz, K. Tamura, H. S. Marcus, R. Donoso, S. Yoshida, and H. J. SWAN, "Measurement of coronary sinus blood flow by continuous thermodilution in man," Circulation, vol. 44, no. 2, pp. 181-195, 1971.

[62] K. M. Bloch, M. Carlsson, H. Arheden, and F. Ståhlberg, "Quantifying coronary sinus flow and global lv perfusion at 3t," BMC medical imaging, vol. 9, no. 1, p. 9, 2009. 
Bibliography

[63] L. Costanzo, Physiology E-Book. Amsterdam, Netherlands: Elsevier Health Sciences, 2017.

[64] F. Perna, E. K. Heist, S. B. Danik, C. D. Barrett, J. N. Ruskin, and M. Mansour, "Assessment of catheter tip contact force resulting in cardiac perforation in swine atria using force sensing technologyclinical perspective," Circulation: Arrhythmia and Electrophysiology, vol. 4, no. 2, pp. 218-224, 2011.

[65] A. Sarkozy, D. Shah, J. Saenen, J. Sieira, T. Phlips, W. Boris, M. Namdar, and C. Vrints, "Contact force in atrial fibrillation: Role of atrial rhythm and ventricular contractions," Circulation: Arrhythmia and Electrophysiology, vol. 8, no. 6, pp. 1342-1350, 2015.

[66] E. Udd and W. Spillman, Fiber Optic Sensors: An Introduction for Engineers and Scientists. Hoboken: Wiley, 2011.

[67] M. Akay, Introduction to Polymer Science and Technology. Bookboon. [Online]. Available: https://books.google.ca/books?id=1J1k7cGYTWcC

[68] T. Srivatsan and T. Sudarshan, Additive Manufacturing: Innovations, Advances, and Applications. Boka Raton: CRC Press, 2015.

[69] (Accessed Jun. 2, 2017). [Online]. Available: https://www.ati-ia.com/app_ content/documents/9620-05-Transducer\%20Section.pdf 